\title{
Treatment of Drug-Resistant Tuberculosis An Official ATS/CDC/ERS/IDSA Clinical Practice Guideline
}

¿ Payam Nahid, Sundari R. Mase, Giovanni Battista Migliori, Giovanni Sotgiu, Graham H. Bothamley, Jan L. Brozek, Adithya Cattamanchi, J. Peter Cegielski, Lisa Chen, Charles L. Daley, Tracy L. Dalton, Raquel Duarte, Federica Fregonese, C. Robert Horsburgh, Jr., Faiz Ahmad Khan, Fayez Kheir, Zhiyi Lan, Alfred Lardizabal, Michael Lauzardo, Joan M. Mangan, Suzanne M. Marks, Lindsay McKenna, Dick Menzies, Carole D. Mitnick, Diana M. Nilsen, Farah Parvez, Charles A. Peloquin, Ann Raftery, H. Simon Schaaf, Neha S. Shah, Jeffrey R. Starke, John W. Wilson, Jonathan M. Wortham, Terence Chorba, and Barbara Seaworth; on behalf of the American Thoracic Society, U.S. Centers for Disease Control and Prevention, European Respiratory Society, and Infectious Diseases Society of America

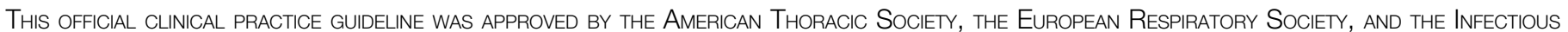
Diseases Society of America September 2019, and was cleared by the U.S. Centers for Disease Control and Prevention September 2019

Background: The American Thoracic Society, U.S. Centers for Disease Control and Prevention, European Respiratory Society, and Infectious Diseases Society of America jointly sponsored this new practice guideline on the treatment of drug-resistant tuberculosis (DR-TB). The document includes recommendations on the treatment of multidrug-resistant TB (MDR-TB) as well as isoniazid-resistant but rifampin-susceptible TB.

Methods: Published systematic reviews, meta-analyses, and a new individual patient data meta-analysis from 12,030 patients, in 50 studies, across 25 countries with confirmed pulmonary rifampinresistant TB were used for this guideline. Meta-analytic approaches included propensity score matching to reduce confounding. Each recommendation was discussed by an expert committee, screened for conflicts of interest, according to the Grading of Recommendations, Assessment, Development, and Evaluation (GRADE) methodology.

Results: Twenty-one Population, Intervention, Comparator, and Outcomes questions were addressed, generating 25 GRADE-based recommendations. Certainty in the evidence was judged to be very low, because the data came from observational studies with significant loss to follow-up and imbalance in background regimens between comparator groups. Good practices in the management of MDR-TB are described. On the basis of the evidence review, a clinical strategy tool for building a treatment regimen for MDR-TB is also provided.

Conclusions: New recommendations are made for the choice and number of drugs in a regimen, the duration of intensive and continuation phases, and the role of injectable drugs for MDR-TB. On the basis of these recommendations, an effective all-oral regimen for MDR-TB can be assembled. Recommendations are also provided on the role of surgery in treatment of MDR-TB and for treatment of contacts exposed to MDR-TB and treatment of isoniazid-resistant TB.

Keywords: MDR-TB; tuberculosis; duration of treatment; drug treatment; treatment monitoring

ORCID IDs: 0000-0003-2811-1311 (P.N.); 0000-0001-5363-0637 (S.R.M.); 0000-0002-2597-574X (G.B.M.); 0000-0002-1600-4474 (G.S.); 0000-0002-7092-8547 (G.H.B.); 0000-0002-3122-0773 (J.B.); 0000-0002-6553-2601 (A.C.); 0000-0001-6804-0111 (L.C.); 0000-0003-3324-926X (C.L.D.); 0000-0001-6838-7895 (C.R.H.); 0000-0003-0473-8734 (F.A.K.); 0000-0002-4192-5080 (F.K.); 0000-0001-5519-2474 (Z.L.); 0000-0003-3273-1097 (A.L.); 0000-0002-7096-4185 (M.L.); 0000-0001-6770-086X (J.M.M.); 0000-0003-3024-1940 (S.M.M.); 0000-0002-4703-0835 (L.M.);

0000-0002-3455-658X (C.D.M.); 0000-0003-1211-5043 (F.P.); 0000-0001-9002-7052 (C.A.P.); 0000-0001-5755-4133 (H.S.S.); 0000-0001-7722-0958 (T.C.); 0000-0003-2922-4940 (B.S.).

Supported by the American Thoracic Society, the United States Centers for Disease Control and Prevention, the European Respiratory Society, and the Infectious Diseases Society of America.

An Executive Summary of this document is available at http://www.atsjournals.org/doi/suppl/10.1164/rccm.201909-1874ST.

aYou may print one copy of this document at no charge. However, if you require more than one copy, you must place a reprint order. Domestic reprint orders: amy.schriver@sheridan.com; international reprint orders: louisa.mott@springer.com.

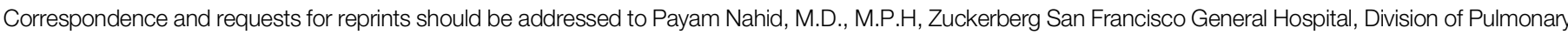

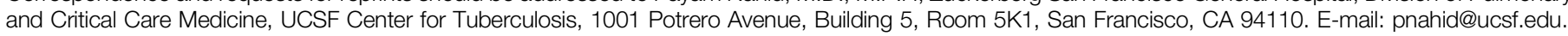

This article has an online supplement, which is accessible from this issue's table of contents at www.atsjournals.org.

Am J Respir Crit Care Med Vol 200, Iss 10, pp e93-e142, Nov 15, 2019

Copyright @ 2019 by the American Thoracic Society

DOI: 10.1164/rccm.201909-1874ST

Internet address: www.atsjournals.org 


\section{Contents \\ Overview \\ Summary of Good Practices \\ Summary of Recommendations \\ Introduction \\ Good Practices for Treating DR-TB \\ Diagnosing TB and Identification of Drug Resistance \\ Treatment and Monitoring of DR- TB \\ Infection Control and DR-TB \\ Case Management for DR-TB \\ Treatment of MDR-TB, Number of \\ Drugs, and Duration of Treatment \\ Phases \\ Number of Drugs in the Regimen \\ Duration of Intensive and \\ Continuation Phases in Treating MDR-TB \\ Drugs and Drug Classes \\ Amoxicillin/Clavulanate \\ Bedaquiline \\ Carbapenems with Clavulanic \\ Acid \\ Clofazimine \\ Cycloserine \\ Delamanid \\ Ethambutol}

\section{Ethionamide and Prothionamide \\ Fluoroquinolones: Levofloxacin, Moxifloxacin, Ciprofloxacin, and Ofloxacin \\ Injectables: Amikacin, Capreomycin, Kanamycin, and Streptomycin \\ Linezolid \\ Macrolides: Azithromycin and \\ Clarithromycin \\ p-Aminosalicylic Acid \\ Pyrazinamide}

Building a Treatment Regimen for MDR-TB

Role of Therapeutic Drug Monitoring in

Treatment of MDR-TB

Shorter-Course, Standardized, 9- to

12-Month Regimen for MDR-TB

Summary of the Evidence

Benefits

Harms

Additional Considerations

Conclusions

Research Needs

Role of Surgery in MDR-TB

Summary of the Evidence

Benefits

Harms
Additional Considerations

Conclusions

Research Needs

Treatment of Isoniazid-Resistant TB

Summary of the Evidence

Benefits

Harms

Additional Considerations

Conclusions

Research Needs

Treatment of MDR-TB in Special

Situations

HIV Infection

Children

Pregnant Women

Treatment of Contacts Exposed to

MDR-TB

Summary of the Evidence

Benefits

Harms

Additional Considerations

Conclusions

Research Needs

Summary of Key Differences

between ATS/CDC/ERS/IDSA and

WHO 2019 Consolidated Guidelines

on Drug-Resistant Tuberculosis

Treatment

\section{Overview}

Treatment of tuberculosis (TB), regardless of the results of drug susceptibility testing (DST), is focused on both curing the individual patient and minimizing the transmission of Mycobacterium tuberculosis to other persons. Thus, effective treatment of TB has benefits for both the individual patient and the community in which the patient resides. However, notable complexities need to be addressed to successfully treat disease resulting from drug-resistant $M$. tuberculosis isolates compared with treatment of drugsusceptible TB disease, including additional molecular and phenotypic diagnostic tests to determine drug susceptibility; the use of second-line drugs, which have toxicities that increase harms that must be balanced with their benefits; and prolonged treatment durations. The new recommendations provided in this guideline are for the treatment of drug-resistant TB (DR-TB), including multidrug-resistant TB (MDR-TB) and isoniazid-resistant $\mathrm{TB}$, and are intended to help providers identify the therapeutic options associated with improved outcomes (i.e., greater treatment success, fewer adverse events, and fewer deaths) and in the context of individual patient values and preferences. Worthy of emphasis, the committee recommends that only drugs to which the patient's M. tuberculosis isolate has documented, or high likelihood of, susceptibility be included in an effective treatment regimen, noted as an ungraded good practice statement, and consistent with ongoing stewardship efforts for the optimal use of antibiotics (1). Drugs known to be ineffective on the basis of in vitro growth-based or molecular DST should not be used. The following alphabetically listed drugs and drug classes were considered for inclusion in treatment regimens: amoxicillin/clavulanate, bedaquiline, carbapenem with clavulanic acid, clofazimine, cycloserine, delamanid, ethambutol, ethionamide, fluoroquinolones, injectable agents, linezolid, macrolides, $p$-aminosalicylic acid, and pyrazinamide. Of note, pretomanid in combination with bedaquiline and linezolid was recently approved by U.S. Food and Drug Administration (FDA) for the treatment of a specific limited population of adults with pulmonary extensively drugresistant (XDR-TB) or treatment-intolerant or nonresponsive MDR-TB; however, the preparation and completion of these guidelines predated this approval (2). For each drug or drug class, the following Population, Intervention, Comparator, and Outcomes (PICO) question was addressed: In patients with MDR-TB, are outcomes safely improved when regimens include the following individual drugs or drug classes compared with regimens that do not include them?

The recommendations in this practice guideline were supported by scientific evidence, including results of a propensity score (PS)-matched individual patient data meta-analyses (IPDMA) conducted using a database of more than 12,000 patient records from 25 countries in support of these guidelines (see APPENDIX A: Methodology in the online supplement) (3). We used the Grading of Recommendations, Assessment, Development, and Evaluation (GRADE) approach to appraise the quality of evidence and to formulate, write, and grade most recommendations $(4,5)$. 
Although published data on costs are noted in these guidelines, the committee did not conduct formal cost-effectiveness analyses of the treatments reviewed to determine a recommendation. The treatment of DR-TB can be complicated and thus is necessarily preceded and accompanied by important components of care relating to the access to TB experts, microbiological and molecular diagnosis, education, monitoring and followup, and global patient-centered strategies. The writing committee considered that these topics are crucial but do not require formal and extensive evidence appraisal in the context of the present guidelines. Following GRADE guidance, it was thus decided that these practices would be addressed in good practice statements. Below, six ungraded good practice statements as well as 25 GRADE-based recommendations addressing 21 PICO questions (Table 1) are listed. Questions were selected according to their importance to clinical practice, as determined by the guideline panel, expert advisors, and patient advocates. The implications of the strength of recommendation, conditional or strong, for patients, clinicians, and policy makers are described in ApPENDIX A and shown in Table 2. Detailed and referenced information on treatment of MDR-TB is available below, including a summary of the evidence, and the benefits, harms, and additional considerations of each practice or recommendation.

\section{Summary of Good Practices}

For patients being evaluated and treated for any form of drug-resistant TB, the following six ungraded good practice statements are emphasized, as the writing committee had high confidence in their net benefit:

1. Consultation should be requested with a TB expert when there is suspicion of or confirmation of DR-TB. In the United States, TB experts can be found through CDC-supported TB Centers of Excellence for Training, Education, and Medical Consultation (http://www. cdc.gov/tb/education/rtmc/default.htm), through local health department TB control programs (https://www.cdc. gov/tb/links/tboffices.htm), and through international MDR-TB expert groups such as the Global TB Network (6).

2. Molecular DSTs should be obtained for rapid detection of mutations associated

Table 1. Questions Regarding the Treatment of Drug-Resistant Tuberculosis Selected by the Guideline Writing Committee

Number of effective drugs in a regimen for MDR-TB

1. Should patients with MDR-TB be prescribed five effective drugs vs. more or fewer agents during the intensive and continuation phases of treatment?

Duration of intensive and continuation phases of treatment for MDR-TB

2. Should patients with MDR-TB undergoing intensive-phase treatment be treated for $\geqslant 6$ mo after culture conversion or $<6$ mo after culture conversion?

3. Should patients with MDR-TB undergoing continuation-phase treatment be treated for $\geqslant 18$ mo after culture conversion or $<18$ mo after culture conversion?

Drug and drug classes for the treatment of MDR-TB

4. In patients with MDR-TB, are outcomes safely improved when regimens include amoxicillin/clavulanate compared with regimens that do not include amoxicillin/clavulanate?

5. In patients with MDR-TB, are outcomes safely improved when regimens include bedaquiline compared with regimens that do not include bedaquiline?

6. In patients with MDR-TB, are outcomes safely improved when regimens include carbapenems with clavulanic acid compared with regimens that do not include them?

7. In patients with MDR-TB, are outcomes safely improved when regimens include clofazimine compared with regimens that do not include clofazimine?

8. In patients with MDR-TB, are outcomes safely improved when regimens include cycloserine compared with regimens that do not include cycloserine?

9. In patients with MDR-TB, are outcomes safely improved when regimens include delamanid compared with regimens that do not include delamanid?

10. In patients with MDR-TB, are outcomes safely improved when regimens include ethambutol compared with regimens that do not include ethambutol?

11. In patients with MDR-TB, are outcomes safely improved when regimens include ethionamide/prothionamide compared with regimens that do not include ethionamide/prothionamide?

12. In patients with MDR-TB, are outcomes safely improved when regimens include fluoroquinolones compared with regimens that do not include fluoroquinolones?

13. In patients with MDR-TB, are outcomes safely improved when regimens include an injectable compared with regimens that do not include an injectable?

14. In patients with MDR-TB, are outcomes safely improved when regimens include linezolid compared with regimens that do not include linezolid?

15. In patients with MDR-TB, are outcomes safely improved when regimens include macrolides compared with regimens that do not include macrolides?

16. In patients with MDR-TB, are outcomes safely improved when regimens include $p$-aminosalicylic acid compared with regimens that do not include $p$-aminosalicylic acid?

17. In patients with MDR-TB, are outcomes safely improved when regimens include pyrazinamide compared with regimens that do not include pyrazinamide?

Use of a standardized, shorter-course regimen of $<12$ mo for the treatment of MDR-TB

18. In patients with MDR-TB, does treatment with a standardized MDR-TB regimen for $\leqslant 12$ mo lead to better outcomes than treatment with an MDR-TB regimen for 18-24 mo?

Treatment of isoniazid-resistant, rifampin-susceptible TB:

19a. Should patients with isoniazid-resistant TB be treated with a regimen composed of a fluoroquinolone, rifampin, ethambutol, and pyrazinamide for 6 mo compared with rifampin, ethambutol, and pyrazinamide (without a fluoroquinolone) for 6 mo?

19b. Should patients with isoniazid-resistant TB be treated with a regimen composed of fluoroquinolone, rifampin, and ethambutol for $6 \mathrm{mo}$ and pyrazinamide for the first 2 mo compared with a regimen composed of a fluoroquinolone, rifampin, ethambutol, and pyrazinamide for 6 mo?

Surgery as adjunctive therapy for MDR-TB:

20. Among patients with MDR/XDR TB receiving antimicrobial therapy, does lung resection surgery (i.e., lobectomy or pneumonectomy) lead to better outcomes than no surgery?

Management of contacts exposed to an infectious patient with MDR-TB:

21. Should contacts exposed to an infectious patient with MDR-TB be offered LTBI treatment vs. followed with observation alone?

Definition of abbreviations: $\mathrm{LTBI}=$ latent TB infection; $\mathrm{MDR}=$ multidrug resistant; $\mathrm{TB}=$ tuberculosis; $\mathrm{XDR}=$ extensively drug resistant.

with resistance. When rifampin resistance is detected, additional DST should be performed immediately for first-line drugs, fluoroquinolones, and aminoglycosides. Resistance to fluoroquinolones should be excluded whenever isoniazid resistance is found.

3. Regimens should include only drugs to which the patient's $M$. tuberculosis isolate has documented or high 
Table 2. Implications of Strong and Conditional Recommendations

Strong Recommendation ("We recommend . . .")
Conditional Recommendation

("We suggest . . .")
For patients

For clinicians

The overwhelming majority of individuals should receive the recommended course of action. Adherence to this recommendation according to the guideline could be used as a quality criterion or performance indicator. Formal decision aids are not likely to be needed to help individuals make decisions consistent with their values and preferences.

For policy makers The recommendation can be adapted as policy in most situations, including for the use as performance indicators.
The majority of individuals in this situation would want the suggested course of action, but a sizeable minority would not.

Different choices will be appropriate for different patients, and you must help each patient arrive at a management decision consistent with her or his values and preferences. Decision aids may be useful to help individuals make decisions consistent with their values and preferences. Clinicians should expect to spend more time with patients when working toward a decision.

Policy making will require substantial debates and involvement of many stakeholders. Policies are also more likely to vary between regions. Performance indicators would have to focus on the fact that adequate deliberation about the management options has taken place. likelihood of susceptibility (hereafter defined as effective). Drugs known to be ineffective based on in vitro growthbased or molecular resistance should NOT be used. This recommendation applies to all drugs and treatment regimens discussed in this practice guideline, unless reliable methods of testing susceptibility for a drug have yet to be developed.

4. Treatment response should be monitored clinically, radiographically, and bacteriologically, with cultures obtained at least monthly for pulmonary TB. When cultures remain positive after 3 months of treatment, susceptibility tests for drugs should be repeated. Weight and other measures of clinical response should be recorded monthly.

5. Patients should be educated and asked about adverse effects at each visit. Adverse effects should be investigated and ameliorated.

6. Patient-centered case management helps patients understand their diagnoses, understand and participate in their treatment, and discuss potential barriers to treatment. Patient-centered strategies and interventions should be used to minimize barriers to treatment.

\section{Summary of Recommendations}

For the selection of an effective MDR-TB treatment regimen and duration of MDRTB treatment:
1. We suggest using at least five drugs in the intensive phase of treatment and four drugs in the continuation phase of treatment (conditional recommendation, very low certainty in the evidence).

2. We suggest an intensive-phase duration of treatment of between 5 and 7 months after culture conversion (conditional recommendation, very low certainty in the evidence).

3. We suggest a total treatment duration of between 15 and 21 months after culture conversion (conditional recommendations, very low certainty in the evidence).

4. In patients with pre-XDR-TB and XDRTB, which are both subsets of MDR-TB, we suggest a total treatment duration of between 15 and 24 months after culture conversion (conditional recommendations, very low certainty in the evidence).

For the selection of oral drugs for MDR-TB treatment (in order of strength of recommendation):

5. We recommend including a latergeneration fluoroquinolone (levofloxacin or moxifloxacin) (strong recommendation, low certainty of evidence).

6. We recommend including bedaquiline (strong recommendation, very low certainty in the evidence).

7. We suggest including linezolid (conditional recommendation, very low certainty in the evidence).
8. We suggest including clofazimine (conditional recommendation, very low certainty of evidence).

9. We suggest including cycloserine (conditional recommendation, very low certainty in the evidence).

10. We suggest including ethambutol only when other more effective drugs cannot be assembled to achieve a total of five drugs in the regimen (conditional recommendation, very low certainty in the evidence).

11. We suggest including pyrazinamide in a regimen for treatment of patients with MDR-TB or with isoniazidresistant TB, when the $M$. tuberculosis isolate has not been found resistant to pyrazinamide (conditional recommendation, very low certainty in the evidence).

12. The guideline panel was unable to make a clinical recommendation for or against delamanid because of the absence of data in the PS-matched IPDMA conducted for this practice guideline. We make a research recommendation for the conduct of randomized clinical trials and cohort studies evaluating the efficacy, safety, and tolerability of delamanid in combination with other oral agents. Until additional data are available, the guideline panel concurs with the conditional recommendation of the 2019 WHO Consolidated Guidelines on Drug-Resistant Tuberculosis 
Treatment that delamanid may be included in the treatment of patients with MDR/rifampin-resistant (RR)-TB aged $\geqslant 3$ years on longer regimens (7).

For selected oral drugs previously included in regimens for the treatment of MDR-TB:

13. We recommend NOT including amoxicillin-clavulanate, with the exception of when the patient is receiving a carbapenem wherein the inclusion of clavulanate is necessary (strong recommendation, very low certainty in the evidence).

14. We recommend NOT including the macrolides azithromycin and clarithromycin (strong recommendation, very low certainty in the evidence).

15. We suggest NOT including ethionamide/prothionamide if more effective drugs are available to construct a regimen with at least five effective drugs (conditional recommendation, very low certainty in the evidence)

16. We suggest NOT including $p$-aminosalicylic acid in a regimen if more effective drugs are available to construct a regimen with at least five effective drugs (conditional recommendation, very low certainty in the evidence).

For the selection of drugs administered through injection when needed to compose an effective treatment regimen for MDR-TB:

17. We suggest including amikacin or streptomycin when susceptibility to these drugs is confirmed (conditional recommendation, very low certainty of evidence).

18. We suggest including a carbapenem (always to be used with amoxicillinclavulanic acid) (conditional recommendation, very low certainty of evidence).

19. We suggest NOT including kanamycin or capreomycin (conditional recommendation, very low certainty in the evidence).

A summary of the recommendations on drugs, the certainty in the evidence, and the relative risks of success and death is provided in Figure 1. Additional details and other outcomes of interest are provided in the section on Drugs and Drug Classes and

\begin{tabular}{|c|c|c|c|c|c|}
\hline \multirow{2}{*}{$\begin{array}{l}\text { Drug / Drug } \\
\text { Class }\end{array}$} & \multicolumn{2}{|c|}{ Recommendation } & \multirow{2}{*}{$\begin{array}{l}\text { Certainty } \\
\text { in the } \\
\text { evidence }\end{array}$} & \multirow{2}{*}{$\begin{array}{c}\text { Relative } \\
(95 \% \mathrm{Cl}) \\
\text { Death }\end{array}$} & \multirow{2}{*}{$\begin{array}{l}\text { Relative } \\
(95 \% \mathrm{Cl}) \\
\text { Success }\end{array}$} \\
\hline & FOR & AGAINST & & & \\
\hline Bedaquiline & Strong & & Very Low & $\begin{array}{c}\text { aOR } 0.4 \\
\text { (0.3 to } 0.5)\end{array}$ & $\begin{array}{c}\text { aOR } 2.0 \\
\text { (1.4 to } 2.9)\end{array}$ \\
\hline $\begin{array}{l}\text { Fluoroquinolone: } \\
\text { Moxifloxacin }\end{array}$ & Strong & & Very Low & $\begin{array}{l}\text { aOR } 0.5 \\
\text { (0.4 to } 0.6)\end{array}$ & $\begin{array}{c}\text { aOR } 3.8 \\
(2.8 \text { to } 5.2)\end{array}$ \\
\hline $\begin{array}{l}\text { Fluoroquinolone: } \\
\text { Levofloxacin }\end{array}$ & Strong & & Very Low & $\begin{array}{l}\text { aOR } 0.6 \\
\text { (0.5 to } 0.7)\end{array}$ & $\begin{array}{c}\text { aOR } 4.2 \\
(3.3 \text { to } 5.4)\end{array}$ \\
\hline Linezolid & Conditional & & Very Low & $\begin{array}{c}\text { aOR } 0.3 \\
\text { (0.2 to } 0.3)\end{array}$ & $\begin{array}{c}\text { aOR } 3.4 \\
\text { (2.6 to } 4.5)\end{array}$ \\
\hline Clofazimine & Conditional & & Very Low & $\begin{array}{c}\text { aOR } 0.8 \\
\text { (0.6 to } 1.0)\end{array}$ & $\begin{array}{c}\text { aOR } 1.5 \\
(1.1 \text { to } 2.1)\end{array}$ \\
\hline Cycloserine & Conditional & & Very Low & $\begin{array}{c}\text { aOR } 0.6 \\
\text { (0.5 to } 0.6)\end{array}$ & $\begin{array}{c}\text { aOR 1.5 } \\
(1.4 \text { to } 1.7)\end{array}$ \\
\hline $\begin{array}{l}\text { Injectables: } \\
\text { Amikacin }\end{array}$ & Conditional & & Very Low & $\begin{array}{c}\text { aOR } 1.0 \\
\text { (0.8 to } 1.2)\end{array}$ & $\begin{array}{c}\text { aOR 2.0 } \\
\text { (1.5 to } 2.6)\end{array}$ \\
\hline $\begin{array}{l}\text { Injectables: } \\
\text { Streptomycin }\end{array}$ & Conditional & & Very Low & $\begin{array}{l}\text { aOR } 0.8 \\
\text { (0.6 to } 1.1)\end{array}$ & $\begin{array}{c}\text { aOR 1.5 } \\
(1.1 \text { to } 2.1)\end{array}$ \\
\hline Ethambutol & Conditional & & Very Low & $\begin{array}{c}\text { aOR } 1.0 \\
\text { (0.9 to } 1.2)\end{array}$ & $\begin{array}{c}\text { aOR } 0.9 \\
\text { (0.7 to } 1.1)\end{array}$ \\
\hline Pyrazinamide & Conditional & & Very Low & $\begin{array}{l}\text { aOR } 0.7 \\
\text { (0.6 to } 0.8)\end{array}$ & $\begin{array}{l}\text { aOR } 0.7 \\
(0.5 \text { to } 0.9)\end{array}$ \\
\hline $\begin{array}{l}\text { Injectables: } \\
\text { Carbapenems w/ } \\
\text { clavulanic acid }\end{array}$ & Conditional & & Very Low & $\begin{array}{c}\text { aOR } 1.0 \\
\text { (0.5 to } 1.7)\end{array}$ & $\begin{array}{c}\text { aOR } 4.0 \\
\text { (1.7 to } 9.1)\end{array}$ \\
\hline Delamanid & $\begin{array}{l}\text { Concur with } \\
\text { WHO conditional } \\
\text { recommendation }\end{array}$ & & & & \\
\hline $\begin{array}{l}\text { Ethionamide } \\
\text { Prothionamide }\end{array}$ & & Conditional & Very Low & $\begin{array}{l}\text { aOR } 0.9 \\
(0.8 \text { to } 1.0)\end{array}$ & $\begin{array}{l}\text { aOR } 0.8 \\
(0.7 \text { to } 0.9)\end{array}$ \\
\hline $\begin{array}{l}\text { Injectables: } \\
\text { Kanamycin }\end{array}$ & & Conditional & Very Low & $\begin{array}{l}\text { aOR } 1.1 \\
\text { (0.9 to } 1.2)\end{array}$ & $\begin{array}{c}\text { aOR } 0.5 \\
(0.4 \text { to } 0.6)\end{array}$ \\
\hline $\begin{array}{l}\text { P-Aminosalicylic } \\
\text { Acid }\end{array}$ & & Conditional & Very Low & $\begin{array}{c}\text { aOR } 1.2 \\
\text { (1.1 to } 1.4)\end{array}$ & $\begin{array}{c}\text { aOR } 0.8 \\
(0.7 \text { to } 1.0)\end{array}$ \\
\hline $\begin{array}{l}\text { Injectables: } \\
\text { Capreomycin }\end{array}$ & & Conditional & Very Low & $\begin{array}{l}\text { aOR } 1.4 \\
\text { (1.1 to } 1.7)\end{array}$ & $\begin{array}{c}\text { aOR } 0.8 \\
(0.6 \text { to } 1.1)\end{array}$ \\
\hline $\begin{array}{l}\text { Macrolides: } \\
\text { Azithromycin } \\
\text { Clarithromycin }\end{array}$ & & Strong & Very Low & $\begin{array}{c}\text { aOR 1.6 } \\
\text { (1.2 to 2.0) }\end{array}$ & $\begin{array}{c}\text { aOR } 0.6 \\
(0.5 \text { to } 0.8)\end{array}$ \\
\hline $\begin{array}{l}\text { Amoxicillin- } \\
\text { clavulanate }\end{array}$ & & Strong & Very Low & $\begin{array}{l}\text { aOR } 1.7 \\
\text { (1.3 to } 2.1)\end{array}$ & $\begin{array}{c}\text { aOR } 0.6 \\
(0.5 \text { to } 0.8)\end{array}$ \\
\hline
\end{tabular}

Figure 1. Summary of recommendations on drugs for use in a treatment regimen for patients with multidrug-resistant tuberculosis, including strength of recommendation, certainty in the evidence, and relative effects on death and treatment success. Additional details and other outcomes of interest are provided in the section on Drugs and Drug Classes, and in APPEndix B: Evidence ProflLes in the online supplement. Success is defined as end of treatment cure or treatment completion. aOR=adjusted odds ratio; $\mathrm{Cl}=$ confidence interval; $\mathrm{WHO}=$ World Health Organization . 
in Appendix B: Evidence Profiles in the online supplement.

For the use of the WHO-recommended standardized shorter-course 9- to 12-month regimen for MDR-TB:

20. The shorter-course regimen is standardized with the use of kanamycin (which the committee recommends against using) and includes drugs for which there is documented or high likelihood of resistance (e.g., isoniazid, ethionamide, pyrazinamide). Although the STREAM (Standard Treatment Regimen of AntiTuberculosis Drugs for Patients with MDR-TB) Stage 1 randomized trial found the shorter-course regimen to be noninferior to longer injectablecontaining regimens with respect to the primary efficacy outcome (8), the guideline committee cannot make a recommendation either for or against this standardized shorter-course regimen, compared with longer individualized all-oral regimens that can be composed in accordance with the recommendations in this practice guideline. We make a research recommendation for the conduct of randomized clinical trials evaluating the efficacy, safety, and tolerability of modified shorter-course regimens that include newer oral agents, exclude injectables, and include drugs for which susceptibility is documented or highly likely.

For the role of surgery in the treatment of MDR-TB:

21. We suggest elective partial lung resection (e.g., lobectomy or wedge resection), rather than medical therapy alone, for adults with MDR-TB receiving antimicrobial-based therapy (conditional recommendation, very low certainty in the evidence). The writing committee believes this option would be beneficial for patients for whom clinical judgement, supported by bacteriological and radiographic data, suggest a strong risk of treatment failure or relapse with medical therapy alone.

22. We suggest medical therapy alone, rather than including elective total lung resection (pneumonectomy), for adults with MDR-TB receiving antimicrobial therapy (conditional recommendation, very low certainty in the evidence). TB:

For the treatment of isoniazid-resistant

23. We suggest adding a later-generation fluoroquinolone to a 6-month regimen of daily rifampin, ethambutol, and pyrazinamide for patients with isoniazid-resistant TB (conditional recommendation, very low certainty in the evidence).

24. In patients with isoniazid-resistant $T B$ treated with a daily regimen of a latergeneration fluoroquinolone, rifampin, ethambutol, and pyrazinamide, we suggest that the duration of pyrazinamide can be shortened to 2 months in selected situations (i.e., noncavitary and lower burden disease or toxicity from pyrazinamide) (conditional recommendation, very low certainty in the evidence).

For the management of contacts to patients with MDR-TB:

25. We suggest offering treatment for latent TB infection (LTBI) for contacts to patients with MDR-TB versus following with observation alone (conditional recommendation, very low certainty in the evidence). We suggest 6 to 12 months of treatment with a later-generation fluoroquinolone alone or with a second drug, on the basis of drug susceptibility of the source-case M. tuberculosis isolate. On the basis of evidence of increased toxicity, adverse events, and discontinuations, pyrazinamide should not be routinely used as the second drug.

In this guideline, we provide new recommendations for treatment of MDR$\mathrm{TB}$ and for treatment of isoniazid-resistant TB. On the basis of the evidence review conducted for this guideline, a clinical strategy tool for building a treatment regimen for MDR-TB is provided. Additional detailed and referenced information on treatment of MDR-TB in special situations (children, pregnant women, and HIV-infected patients), approaches to treatment monitoring, the use of case management and directly observed therapy, the role of therapeutic drug monitoring, and key research priorities are provided below.

\section{Introduction}

The American Thoracic Society (ATS), U.S Centers for Disease Control and Prevention, European Respiratory Society (ERS), and Infectious Diseases Society of America (IDSA) have jointly developed these DrugResistant Tuberculosis guidelines. These new recommendations are based on the certainty in the evidence (also known as the quality of evidence) and developed based on the evidence that was appraised using GRADE methodology, which incorporates patient values and costs as well as judgments about trade-offs between benefits and harms $(4,5)$. A carefully selected panel of experts, screened for conflicts of interest, including specialists in pulmonary medicine, infectious diseases, pediatrics, primary care, public health, epidemiology, economics, pharmacokinetics, microbiology, systematic review methodology, and patient advocacy, was assembled to assess the evidence supporting each recommendation. In contrast to prior analytic approaches, wherein systematic reviews of aggregate data were used for decision-making, a new PS-matched meta-analysis of IPDMA from 12,030 patients, in 50 studies, from 25 countries with confirmed pulmonary RR-TB was conducted for this guideline (3). Given the paucity of high-quality randomized controlled trials conducted in DR-TB, individual data from observational studies represent the next best level of evidence for analyses. Nonetheless, the writing committee noted that observational data are prone to bias and confounding. The 21 PICO questions and associated recommendations are summarized below, all appraised using GRADE methodology (see ApPEndix B). Questions were selected according to their importance to clinical practice, as determined by the guideline panel, expert advisors, and patient advocates. The implications of the strength of recommendation, conditional or strong, for patients, clinicians, and policy makers are described in Appendix A. On the basis of the GRADE methodology framework, all recommendations in these guidelines are based on very low certainty in the evidence. The writing committee selected death, treatment success, and serious adverse effects as the endpoints of critical importance on which to generate recommendations. Our meta-analytic approaches included PS matching to 
reduce confounding (on the basis of individual-level covariates of age, sex, HIV coinfection, acid-fast bacilli [AFB] smear results, cavitation on chest radiographs, history of TB treatment with first-line or second-line TB drugs, and number of possibly effective drugs in the regimen, among other variables), described in detail in the publication of the PS-matched IPDMA publication and in APPENDIX A (3). However, the risk of bias remained serious, because the average loss to follow-up across included studies was $10 \%$ to $20 \%$. In addition, despite the efforts, there was a large residual imbalance in background regimens used in experimental and control groups.

These guidelines are intended for settings in which treatment is individualized and where mycobacterial cultures, molecular (genotypic) and culture-based (phenotypic) DSTs, and radiographic facilities are available $(9,10)$. Of note, published data on costs are referenced in these guidelines, but the committee did not conduct formal costeffectiveness analyses of the treatments and interventions reviewed in relation to determining a recommendation.

In these guidelines, MDR-TB is defined specifically as resistance to at least isoniazid and rifampin, the two most important first-line drugs. XDR-TB is a subset of MDR-TB with additional resistance to a fluoroquinolone and a second-line injectable agent. Because XDR-TB evolves from MDR-TB in two steps, the term "pre-XDRTB" was introduced to identify MDR-TB with additional resistance to either one but not both of these classes of drugs. In these guidelines, we also provide recommendations for the treatment of isoniazid-resistant TB.

\section{Good Practices for Treating DR-TB}

The fundamentals of TB care, regardless of the treatment selected, rest on ensuring timely diagnosis and initiation of appropriate therapy, with ongoing support and management to achieve successful treatment completion and cure. The responsibility for successful treatment of TB is placed primarily on the provider or program initiating therapy rather than on the patient (11). Nevertheless, a patientcentered approach, described more fully in the section on case management below, requires the involvement of the patient in decision-making. We recommend seeking consultation with an expert in $\mathrm{TB}$ when there is suspicion for or confirmation of DR-TB (ungraded good practice statement). In the United States, DR-TB experts can be found through CDCsupported TB Centers of Excellence for Training, Education, and Medical Consultation (http://www.cdc.gov/ tb/education/rtmc/default.htm), through local health department TB Control Programs (https://www.cdc.gov/tb/ links/tboffices.htm), and through international MDR-TB expert groups such as the British Thoracic Society MDR-TB Clinical Advisory Service (http://mdrtb. brit-thoracic.org.uk/) and the Global TB Network (6). Additional good practices in the treatment of patients in need of evaluation for DR-TB include the following:

\section{Diagnosing TB and Identification of Drug Resistance}

The potential for drug resistance is considered in every patient. An aggressive effort is made to collect biological specimens for detection of $M$. tuberculosis and for drug resistance. A rapid test for a least rifampin resistance should ideally be done for every patient, but especially for those at risk of drug resistance. The concern for possible resistance is heightened for patients from areas of the world with at least a moderate incidence of TB in general $(\geqslant 20 / 100,000)$ and a high primary MDR-TB prevalence $(\geqslant 2 \%)(12)$. Individuals who have or recently had close contact with a patient with infectious DR-TB, especially when the contact is a young child or has HIV infection, are at risk of developing DR-TB. Molecular methods, and more recently whole-genome sequencing (WGS), are increasingly available and can provide information on resistance to all first-line and many second-line drugs. Many public health laboratories provide molecular tests, and WGS is available in selected laboratories. These tests can be used to guide initial therapeutic decisions and contribute to population-level control of

DR-TB. Providers should be familiar with phenotypic and genotypic laboratory services available in their locale. In the United States, CDC's Division of Tuberculosis Elimination Laboratory Branch provides testing services for both clinical specimens and isolates of M. tuberculosis (https://www.cdc. gov/tb/topic/laboratory/default.htm). CDC's Molecular Detection of Drug
Resistance (MDDR) service serves to rapidly identify DR-TB. This service uses DNA sequencing for detection of mutations most frequently associated with resistance to both first-line (e.g., rifampin, isoniazid, ethambutol, and pyrazinamide) as well as second-line drugs (MDDR Service Request Form is available here: https:// www.cdc.gov/tb/topic/laboratory/ MDDRsubmissionform.pdf). Recently published ATS/CDC/IDSA Official Practice Guidelines for the diagnosis of TB provide additional details on the optimal use of diagnostic tools and algorithms (13).

\section{Treatment and Monitoring of DR-TB}

Regimens should only include drugs to which the patient's isolate has documented or high likelihood of susceptibility. Drugs known to be ineffective, on the basis of in vitro resistance or clinical and epidemiological information (i.e., resistance in the index case or high population prevalence of resistance), should not be used, even when resistance is present in only a small percentage of the mycobacteria in the population. If at least $1 \%$ of organisms in a solid media culture exhibit resistance to a drug (the current standard laboratory definition of drug resistance) (14), using that drug in a regimen will increase the risk for poor treatment outcomes, and the isolate will eventually exhibit $100 \%$ resistance to the drug. Drugs should be selected based on their efficacy and the likelihood that patients will be able to tolerate them without significant toxicity. Treatment response is monitored clinically (decrease in cough and systemic symptoms and increase in weight), radiographically, and bacteriologically (15-18). If sputum cultures remain positive after 3 months of treatment, or if there is bacteriological reversion from negative to positive at any time, DST should be repeated (11). Patients should be asked about the clinical response at each visit and weight recorded monthly. Monthly cultures help to identify early evidence of failure (19). Most persons have difficulty taking one or more of the drugs used to treat MDR-TB. Patients should be educated about adverse effects, and all adverse effects should be investigated and ameliorated. Some adverse effects are difficult to tolerate but do not put patients at risk for serious short- or long-term damage to organ systems and can be managed with symptom-specific ancillary medication and supportive care. Nausea 
with vomiting is common and is not always an indication to discontinue therapy permanently. New-onset vomiting may indicate liver toxicity or, in children especially, increased intracranial pressure. If drug-induced liver toxicity (and increased intracranial pressure) is excluded, vomiting can be managed by changing dosing schedule, giving medications with a small snack (noting that this may affect plasma concentrations of the drug), or premedicating adult patients with an antiemetic (noting that some prolong the QT interval) before the dose. Patients may note fatigue or describe myalgia or arthralgia, but these symptoms are not typically treatment limiting. Although low-grade adverse effects can resolve gradually over time, recognizing the negative impact on patient's quality of life, all adverse effects must be addressed diligently. The Curry International TB Center's guides, Drug Resistant TB: Clinician's Survival Guide and the Nursing Guide for Managing Side Effects to DrugResistant TB Treatment, in addition to the World Health Organization (WHO) companion handbook, are good resources to assist in evaluation and management of patients $(15,16,18)$.

\section{Infection Control and DR-TB}

Three main strategies will reduce the transmission of DR-TB: rapid diagnosis, prompt appropriate treatment, and improved airborne infection control $(11,13,20,21)$. Rapid molecular DST and conventional phenotypic culture-based DST are almost universally available in the United States, Europe, and low-incidence, high-resource countries (22). Targeted active case finding combined with rapid diagnostics leading to effective therapy is a strategy endorsed by WHO $(13,23,24)$.

Treatment delays have been associated with increased transmission. A systematic review and meta-analysis looking at patientrelated risk factors for transmission of $M$. tuberculosis found that treatment initiation delays of 28 to 30 days were significantly associated with increased transmission to contacts (25). Effective therapy renders patients with $\mathrm{TB}$, even those with DR-TB, rapidly noninfectious $(24,26)$. The rapid reduction in infectiousness even in the setting of MDR-TB makes outpatient therapy possible, but directly observed therapy (DOT) applied through patientcentered approaches plays an important role in this regard $(11,23)$. Infection control measures such as administrative and environmental controls, and personal protective equipment, listed in order of priority, are important for preventing the transmission of $M$. tuberculosis regardless of drug susceptibility. Every healthcare facility should have these measures in place per CDC guidelines (27). Furthermore, patients should be educated about the importance of infection control measures, such as the value of good ventilation, open windows, and the risks of exposure for children $<5$ years of age and immunocompromised individuals (21).

\section{Case Management for DR-TB}

Case management is a collaborative process that entails engaging with patients; comprehensively assessing, monitoring, and attending to patients' physical, psychological, social, material, and informational needs; care planning; medication management; facilitating access to services; and functioning as patient advocate/agent (28-30). Commonly, case management is used in community and public health settings to coordinate services for patients with chronic and complex health conditions and to attain good, quality, cost-effective outcomes. The practice of case management has long been considered an important component of care for patients with $\operatorname{TB}(20,31,32)$, and a patient-centered (or family-centered in case of children) approach is preferred $(7,11$, 33). Patient-centered case management helps patients understand their diagnosis and treatment and participate in treatment selection and promotes communication about factors that matter to the patient (33-35). Importantly, a patient-centered approach includes discussions with the patient to identify potential barriers to care and the selection of strategies and interventions to address and minimize these barriers $(7,11,33$, 35-37).

Case management tools for drugresistant TB include a drug-o-gram, which organizes clinical details in a format that aligns test results with treatment, as well as laboratory, bacteriology, and other toxicity monitoring flow sheets. This format is a visible representation of pertinent clinical parameters being tracked $(16,38)$. A monitoring checklist or care plan can also help the case manager ensure timely drug toxicity monitoring and provision of examinations required to assess a patient's response to treatment. Case management interventions that have demonstrated favorable outcomes include the provision of patient education and counseling related to diagnosis, treatment, and adherence, as well as the use of treatment adherence interventions alongside suitable patientcentered administration options. For example, home or community-based DOT is shown to be preferred and associated with greater likelihood of treatment success compared with health facility-based DOT or self-administered therapy $(11,39)$. Enhancing treatment completion through the use of patient-centered case management strategies, including DOT, also aims to reduce risk of acquisition of drug resistance, which aligns with international efforts in antibiotic stewardship (1). Recent WHO guidelines evaluated various adherence interventions and outcomes of TB treatment through a systematic review of clinical trials and observational studies, identifying data from 129 studies for quantitative analysis published through 2018 (39). Another meta-analysis of 22 randomized controlled trials of DOT and other interventions to improve adherence reported significant increases in cure with DOT (18\%) and with patient education and counseling (16\%). Compared with the complementary groups, loss to follow-up was $49 \%$ lower with DOT, $26 \%$ lower with financial incentives, and $13 \%$ lower with patient education and counseling. However, there was no significant reduction in mortality (40).

The use of video-enabled electronic devices to conduct DOT is expanding rapidly $(41,42)$. Electronic methods for DOT have the potential to improve TB treatment outcomes and extend public health support to patients with TB when face-to-face DOT is not feasible. Pilot studies have reported that patients find electronic methods for DOT to be both acceptable and more convenient than traditional in-person DOT. These studies also reported good adherence, fewer unobserved doses, and high satisfaction among study participants (43-46). Further evidence is needed to validate electronically observed therapy under more diverse conditions, with larger cross-sections of patient subgroups, including children, and to determine to what degree these methods impact treatment outcomes for patients with DR-TB (39, 47-49). 


\section{Treatment of MDR-TB, Number of Drugs, and Duration of Treatment Phases}

\section{Number of Drugs in the Regimen}

PICO Question 1: Should patients with MDR-TB be prescribed five effective drugs versus more or fewer agents during the intensive and continuation phases of treatment?

Recommendation 1a: We suggest using at least five drugs in the intensive phase of treatment of MDRTB (conditional recommendation, very low certainty of evidence).

Recommendation 1b: We suggest using at least four drugs in the continuation phase of treatment of MDR-TB (conditional recommendation, very low certainty of evidence).

Up until the recent 2019 WHO

Consolidated Guidelines on Drug-Resistant Tuberculosis Treatment (7), WHO has recommended at least five drugs in the intensive phase of treatment, defined by the use of a second-line injectable agent (23). The recent WHO change to recommending at least four effective drugs at initiation of treatment is graded as a conditional recommendation with very low certainty in the estimates of effect (7). Of note, both our guideline committee and the 2019 WHO guidelines promote the use of newer or repurposed oral agents with greater efficacy and deemphasize the use of injectable agents (7). Given these changes and that an injectable drug is no longer obligatory, the intensive phase can no longer be defined by the inclusion of injectables. In this guideline, we define the intensive phase to be the initiation phase of treatment with at least five effective drugs. These recommendations do not apply to the WHO-endorsed shorter-course 9- to 12-month (Bangladesh) regimen, which combines seven drugs for 4 months or until sputum smear conversion, whichever is the longer period, followed by four drugs in the continuation phase (23).

Summary of the evidence. Procedures and methodology to assemble and rank the certainty in the evidence are reported in Appendix A, with evidence profiles for PICO questions reported in APPENDIX B. For the analysis of number of effective drugs, we counted drugs with published evidence from randomized trials of effectiveness (3). Hence, we counted ethambutol, pyrazinamide, all injectables and fluoroquinolones, ethionamide/prothionamide, cycloserine/terizidone, and $p$-aminosalicylic acid on the basis of DST showing susceptibility and counted clofazimine, linezolid, carbapenems, bedaquiline, and delamanid-if susceptible, or no DST for that drug. We did not count amoxicillin/clavulanate (in the absence of a carbapenem) and macrolides as effective drugs (see PICO Questions 4 and 15). The intensive phase was defined by the use of an injectable agent (other than imipenem-cilastatin or meropenem). Culture conversion was not an outcome measure. Instead, the analysis assessed the association of the number of possibly effective drugs included in the first 2 weeks of the intensive phase with the two final treatment outcomes: 1) "treatment success," which includes both cure and treatment completed; and 2) mortality. For the intensive phase of treatment, final treatment outcomes were compared between those treated with five or more $(n=2,527)$ effective drugs and those treated with three or four effective drugs $(n=5,923)$.

Benefits. In our PS-matched IPDMA, using zero to two effective drugs as the reference value, treatment success was most likely with regimens for MDR-TB containing five effective drugs in the intensive phase (Table 3). Mortality was also significantly reduced for those taking five or six effective drugs.

The number of drugs used in the continuation phase was also evaluated (Table 4). In the continuation phase, four

Table 3. Propensity Score-matched Analysis of the Number of Drugs in the Intensive Phase of Treatment and the aOR of Treatment Success versus Failure or Relapse

No. of Patients

No. of Drugs
Success/Total (\%) Death/Total (\%)
Propensity Score-matched Analysis

Risk Difference

aOR $(95 \% \mathrm{Cl}) \quad$ (95\% Cl) $(\%)$

For the analysis of success vs. fail ${ }^{*} /$ relapse $^{\dagger}$

0-2 drugs

3 drugs

4 drugs

5 drugs

$\geqslant 6$ drugs

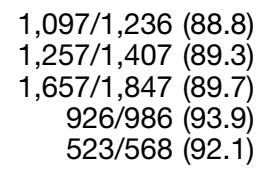

$1,097 / 1,236(88.8)$
$1,257 / 1,407(89.3)$
$1,657 / 1,847(89.7)$
$926 / 986(93.9)$
$523 / 568(92.1)$

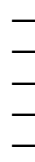

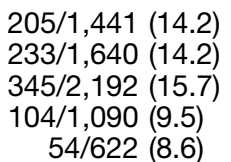

1.0 (reference)
$1.7(1.4$ to 2.0$)$
$1.2(1.4$ to 2.0$)$
$3.0(2.3$ to 3.9$)$
$2.3(1.6$ to 3.1$)$

6 (4 to 7 )

8 (6 to 9$)$

8 (7 to 10)

4 (1 to 7 )

For the analysis of death vs. success $/$ fail $^{\star} /$ relapse $^{\dagger}$
0-2 drugs
3 drugs
4 drugs
5 drugs
$\geqslant 6$ drugs

$\begin{array}{cc}- & 205 / 1,441(14.2) \\ - & 233 / 1,640(14.2) \\ - & 345 / 2,192(15.7) \\ - & 104 / 1,090(9.5) \\ - & 54 / 622(8.6)\end{array}$

1.0 (reference)

0.9 (0.8 to 1.1)

$1.1(0.9$ to 1.2$)$

0.6 (0.5 to 0.7$)$

$0.5(0.4$ to 0.7$)$

Definition of abbreviations: aOR=adjusted odds ratio; $\mathrm{Cl}=$ confidence interval.

*World Health Organization definitions: fail = treatment terminated or need for permanent regimen change of at least two antituberculosis drugs because of: lack of conversion by the end of the intensive phase, bacteriological reversion in the continuation phase after conversion to negative, evidence of additional acquired resistance to fluoroquinolones or second-line injectable drugs or adverse drug reactions.

${ }^{\dagger}$ Relapse was defined as a positive bacteriological culture in the 12 months after treatment completion. 
Table 4. Propensity Score-matched Analysis of the Number of Drugs in the Continuation Phase of Treatment and the aOR of Treatment Success versus Failure or Relapse

No. of Patients

No. of Drugs

\section{Success/Total (\%)}

Death/Total (\%)

For the analysis of success vs. fail ${ }^{\star} /$ relapse $^{\dagger}$

$$
\begin{aligned}
& 0-1 \text { drug } \\
& 2 \text { drugs } \\
& 3 \text { drugs } \\
& 4 \text { drugs } \\
& \geqslant 5 \text { drugs }
\end{aligned}
$$

Propensity Score-matched Analysis

Risk Difference

For the analysis of death vs. success/fail ${ }^{\star} /$ relapse $^{\dagger}$

$\begin{array}{lcc}0-1 \text { drug } & - & 187 / 1,331(14.0) \\ 2 \text { drugs } & - & 193 / 1,618(11.9) \\ 3 \text { drugs } & - & 307 / 2,117(14.5) \\ 4 \text { drugs } & - & 78 / 942(8.3) \\ \geqslant 5 \text { drugs } & - & 37 / 420(8.8)\end{array}$

1.0 (reference)
$1.1(0.9$ to 1.3$)$
$1.2(1.0$ to 1.4$)$
$2.3(1.7$ to 3.1$)$
$1.2(0.9$ to 1.8$)$

$1(-1$ to 3$)$

3 (1 to 5$)$

3 (1 to 5$)$

$-4(-8$ to -1$)$

Definition of abbreviations: aOR = adjusted odds ratio; $\mathrm{Cl}=$ confidence interval.

*World Health Organization definitions: fail = treatment terminated or need for permanent regimen change of at least two anti-tuberculosis drugs because of: lack of conversion by the end of the intensive phase, bacteriological reversion in the continuation phase after conversion to negative, evidence of additional acquired resistance to fluoroquinolones or second-line injectable drugs or adverse drug reactions.

${ }^{\dagger}$ Relapse was reported as a positive bacteriological culture in the 12 months after treatment completion.

drugs gave the greatest success (adjusted odds ratio $[\mathrm{aOR}], 2.3$ ) and four or more drugs the greatest reduction in mortality.

Few significant differences were found in patients with pre-XDR-TB or XDR-TB. Subgroup analyses in pre-XDR-TB and XDR-TB did not suggest that a different number of effective drugs for the intensive and continuation phases was required to achieve good outcomes.

Harms. The recommendation places a higher value on reduced morbidity and greater treatment success than on the as yet unmeasured avoidance of the summative or synergistic toxicity across drugs in the treatment regimen.

Additional considerations. Each drug was counted as equivalent in effectiveness, representing the average effectiveness of the most widely used MDR-TB drugs, for the purpose of adjusting for the number of other effective drugs in the regimen. A regimen composition approach with suggested ranking of drugs is provided in the section Building a Treatment Regimen for MDRTB. The smallest number of drugs to achieve sputum conversion and ensure a cure without relapse minimizes problems with drug-drug interactions, reduces adverse effects, and is cost effective. Injectable drugs are problematic in terms of route of administration, patient preference, significant adverse effects such as hearing loss or vertigo, and costs of administration and monitoring blood levels. On the basis of these limitations, combined with evidence of low efficacy for injectable agents, the use of injectables in defining the intensive phase of treatment is no longer appropriate. WHO has recategorized injectables to a lower grouping and recommended that the injectables only be used when oral medicines from higher categories cannot be used (7). The South African National Department of Health also has endorsed replacing the injectable agents in the shorter-course MDR-TB treatment regimen in adults and children $>12$ years of age with bedaquiline, on the basis of a retrospective observational study of improved mortality with adding bedaquiline (50). In conjunction, experts from the Sentinel Project on Pediatric Drug-Resistant Tuberculosis (http://sentinel-project.org) have also called for replacing the injectable drug in children $<12$ years of age with an alternative effective drug (51).

Conclusions. At least five drugs should be used in the intensive phase of treatment and four drugs in the continuation phase of treatment of MDR-TB (conditional recommendation, very low certainty of evidence). Drugs of poor or doubtful efficacy should not be added to a regimen purely to ensure that the recommended number of drugs is obtained.

Research needs. Randomized controlled trials with fewer but more effective and safer drugs should be undertaken (e.g., TB-PRACTECAL, [Pragmatic Clinical Trial for a More Effective Concise and Less Toxic MDR-TB Treatment Regimen] ClinicalTrials.gov identifier NCT02589782). Randomized trials comparing regimens with and without injectable agents are underway (e.g., STREAM-stage 2, NCT02409290; Evaluating Newly Approved Drugs for Multidrug-resistant TB: endTB, NCT02754765). The effect of duration of individual drugs in the intensive phase on culture conversion as well as treatment cure without relapse should be explored (52).

\section{Duration of Intensive and Continuation Phases in Treating MDR-TB}

Treatment of both drug-susceptible and MDR-TB has typically been divided across

PICO Question 2: Should patients with MDR-TB undergoing intensive-phase treatment be treated for $\geqslant 6$ months after culture conversion or $<6$ months after culture conversion?

Recommendation 2: In patients with MDR-TB, we suggest an intensivephase duration of treatment of between 5 and 7 months after culture conversion (conditional recommendation, very low certainty of evidence). 
two phases, with an initial "intensive" phase that contains more drugs than the subsequent "continuation" phase. For MDR-TB, this intensive phase has historically been characterized by the use of an aminoglycoside (amikacin or kanamycin) or a polypeptide (capreomycin) delivered parenterally (53). This approach has been intended to provide greater bactericidal activity during the time when the bacillary burden is highest and reducing the number of drugs in the continuation phase to reduce risk of toxicity and intolerability caused by the multidrug regimen at a phase when the microbial burden has diminished. The 6-month duration of bedaquiline treatment, in part, was developed by analogy, and clinical trials are underway to determine whether this drug may replace aminoglycosides in terms of an initial intensive phase of treatment. The duration of the intensive phase has never been examined through a randomized, controlled clinical trial. Rather, it has been defined by a combination of practicality, the clinical experiences of MDR-TB experts, and, most recently, an IPDMA that resulted in the WHO practice guideline recommendations of 2011 that an intensive phase of at least 8 months' duration be used (54). This represented a departure from the prior recommendations in 2008 that the injectable agent should be continued for at least 6 months and at least 4 months after the patient first becomes and remains smear or culture negative (55). This is similar to current expert guidance provided by the Curry International TB Center Drug Resistant TB: Clinician's Survival Guide, namely "Intensive phase: recommend at least 6 months beyond culture conversion for the use of injectable agent" (16). The PS-matched IPDMA completed as part of the present guideline development process includes cohorts that were treated according to this range of durations of the intensive phase and represents the available evidence base for our recommendation. Our analyses and recommendations for the duration of intensive and continuation phases of therapy are anchored to the timing of culture conversion, as this approach factors in that treatment response may vary by patient, resistance patterns, and regimen composition and potency, among other factors.

Summary of the evidence. Procedures and methodology to assemble and rank the certainty in the evidence are reported in Appendix A, with evidence profiles for PICO questions reported in Appendix B. The association between treatment duration of the intensive phase (defined by the use of an injectable agent) after culture conversion and outcomes (success, failure, relapse) was examined among 4,122 (49.3\%) subjects from 29 studies who had sufficient information to be included in this analysis (3). This total reflected exclusion of studies without a reported intensive-phase duration $(n=942$ [11.3\%] patients) or time to culture conversion ( $n=1,880$ [22.5\%] patients), as well as 955 (11.4\%) patients in the included studies but for whom intensive-phase duration or time to conversion was not known. Patients taking the 9- to 12-month standardized shorter-course regimen were excluded (see PICO Question 18). An additional 464 (5.0\%) patients were excluded for conversion after the end of the intensive phase or after 14.3 months, or for an intensive phase exceeding 24.3 months. Among the 4,122 patients included, 3,303 (80.1\%) had MDR$\mathrm{TB}$ without resistance to fluoroquinolone or second-line injectable. The remainder had pre- or XDR-TB. To reflect meaningful variations in the duration of the intensive regimen after conversion, stratified analyses were conducted: strata were 0 to 1.0 months, 1.01 to 3.0 months, 3.01 to 5.0 months, 5.01 to 7.0 months, and 7.1 to 15.0 months (see Table 5). The largest proportion (28.6\% $[1,179])$ received treatment for 5.01 to 7 (mean, 5.9) months after conversion. Time to conversion was roughly inversely proportional to duration of intensive phase, suggesting that the tendency in the included studies was to treat for a total intensive-phase duration of 5 to 8 months.

Benefits. The patients who received treatment for 5.01 to 7 months after conversion experienced a 3.3-fold increase in adjusted odds of treatment success (95\% confidence interval [CI], 2.1-5.2) compared with the reference group (0-1.0 months of intensive phase after conversion). Some variability was observed in distribution of intensive-phase duration after culture conversion by baseline characteristics: $<10.0 \%$ of patients who received longer durations of intensive phase were HIVcoinfected, compared with $>15 \%$ in the 0.0 to 3.0 month interval groups. Number of (effective) drugs was greater in longer durations than in shorter.

Patients with MDR-TB who received 5.01 to 7.0 months of intensive-phase treatment after conversion had the highest odds of success in adjusted PS-matched analysis; although other, shorter intervals showed improvement over the reference, $\leqslant 1.0$ month of postconversion treatment, the benefits of 5 to 7 months of treatment after conversion were more pronounced (Table 6). The effect estimates were also better for subgroups of MDR only (aOR, 2.0; 95\% CI, 1.1-3.4) and pre-XDR (aOR, 1.5; 95\% CI, 0.6-3.7) subgroups.

\section{Harms}

Detailed data on duration-related toxicities were not available through our PS-matched IPDMA. On the basis of the clinical observations and experiences of the MDRTB experts on the guideline committee, the intensive phase should not be prolonged beyond that considered necessary to optimize treatment outcomes.

Additional considerations. No information is available in the datasets on

Table 5. Distribution of 4,122 Patients by Duration of Intensive Phase after Culture Conversion

\section{Interval from Culture Conversion to \\ End of Intensive Phase (Mean) (mo)}

No. of Patients (\%)

Mean Time to
Conversion (mo)
Mean Total Intensive
Phase Duration (mo)
Mean Total Treatment Duration (mo)
$0-1.0(0.6)$

$1.01-3.0(2.3)$

$3.01-5.0(4.2)$

$5.01-7.0(5.9)$

7.01-15.0 (10.5)

$251(6.1)$
$695(16.9)$
$917(22.2)$
$1,179(28.6)$
$1,080(26.2)$

4.7

3.0

2.1

1.7

2.0
5.3

5.3

6.4

7.6

12.5
20.0

20.1

20.0

20.6

22.9 
Table 6. Adjusted Estimates of Treatment Success by Duration of Intensive Phase after Culture Conversion, All Forms of Multidrug-Resistant Tuberculosis $(N=4,122)$

\begin{tabular}{|c|c|c|c|c|c|c|}
\hline \multirow{2}{*}{$\begin{array}{l}\text { Intervals from Sputum Culture } \\
\text { Conversion to End of } \\
\text { Intensive-Phase Treatment (mo) }\end{array}$} & \multicolumn{2}{|c|}{ No. of Patients } & \multicolumn{4}{|c|}{ Propensity Score-matched Analysis } \\
\hline & $\begin{array}{l}\text { Treatment } \\
\text { Success }\end{array}$ & Total & No. of Pairs & aOR & $95 \% \mathrm{Cl}$ & $\begin{array}{l}\text { Risk Difference } \\
(95 \% \mathrm{Cl})\end{array}$ \\
\hline $0-1.0$ & 239 & 251 & - & 1.0 & Reference & - \\
\hline $1.01-3.0$ & 668 & 695 & 694 & 1.5 & 1.0 to 2.3 & 0.02 (0.00 to 0.03$)$ \\
\hline $3.01-5.0$ & 878 & 917 & 906 & 1.4 & 1.0 to 2.0 & 0.02 (0.00 to 0.03$)$ \\
\hline $5.01-7.0$ & 1,158 & 1,179 & 1,179 & 3.3 & 2.1 to 5.2 & $0.04(0.03$ to 0.05$)$ \\
\hline $7.01-15.0$ & 1,025 & 1,080 & 1,079 & 1.1 & 0.8 to 1.5 & $0.01(-0.01$ to 0.02$)$ \\
\hline
\end{tabular}

Definition of abbreviations: $\mathrm{aOR}=$ adjusted odds ratio; $\mathrm{Cl}=$ confidence interval.

how duration was selected. Duration selected may reflect interim response to treatment or may reflect a planned duration that is not conditioned on treatment response; the latter is suggested by the observation that duration was inversely related to time to conversion except in the 7.01- to 15-month interval. Analyses were adjusted for possible baseline confounders, relying on PS matching, to reduce the bias introduced. However, the possibility of unmeasured confounding by indication, in particular by time-varying characteristics (such as toxicity, microbiological, radiographic, or clinical results), cannot be ruled out. Such confounding by indication would likely result in an underestimate of the benefit of a longer intensive phase after conversion: patients in the 7.01- to 15 -month interval had slower conversion and more poor outcomes than those in the 5.01- to 7.0month interval. Last, the optimal total duration of treatment for MDR-TB using injectable-free, all-oral regimens cannot be determined from these datasets, but clinical trials evaluating newer drugs and all-oral regimens for MDR-TB are underway (56).

Conclusions. We suggest an intensivephase treatment of between 5 and 7 months after culture conversion in patients with MDR-TB (conditional recommendation, very low certainty in the evidence). The clinical context, extent of disease, and response to treatment, among other factors, will play a role in choosing a final duration from within the recommended range. There were limited data in pre-XDR-TB and XDRTB. Subgroup analyses in pre-XDR and XDR-TB did not suggest that a different duration for the intensive phase would be required to achieve good outcomes. This intensive-phase duration recommendation does not apply to the 9- to 12-month shortercourse regimen addressed in PICO 18.
Research needs. Further research is urgently needed to define the optimal durations of treatment using newer drugs and all-oral regimens. Research in defining optimal duration that prevents death or loss to follow-up is also needed. In the short term, further research on datasets that include longitudinal observations that can inform choice of regimen duration would be important to reducing the uncertainty around the present recommendations. In the long term, randomized controlled trials evaluating various intensive phases and durations are needed. Research on stratified medicine approaches that use measures of burden of disease and consider subgroups in the selection of the optimal duration may allow for greater precision and better inform decision-making around the balance of benefits and harms of durations for individual patients (57).

Summary of the evidence. Procedures and methodology to assemble and rank the certainty in the evidence are reported in APPENDIX A, with evidence profiles for PICO questions reported in APPENDIX B. The association between total duration of treatment after culture conversion and outcomes (success, failure, relapse) was performed on 4,691 observations from 32 studies (3). We excluded 18 studies $(n=2,615)$ that did not report time to culture conversion and 798 patients from included studies because endpoints were missing. Last, 259 patients were excluded for outlier values in total duration or culture conversion; patients whose outcome was loss to follow-up or death were excluded. Among the 4,691 included, 3,034 had MDR-TB. The remainder had pre-XDR or XDR-TB. To reflect meaningful variations in the duration of treatment after conversion, stratified analyses were conducted: Time to conversion was inversely related to duration of treatment after conversion (Table 7).

Benefits. Among all resistance groups, durations of 15.01 to 21 months after conversion outperformed the reference (12.01-15 mo); intervals of 15.01 to 18 (aOR, 2.1; 95\% CI, 1.4-3.1), 18.01 to 21 (aOR, 1.6; 95\% CI, 1.1-2.3), and 21.01 to 24 (aOR, 1.2; 95\% CI, 0.9-1.8) months were indistinguishable from each other (Table 8). In the MDR-only subgroup, the duration of 15.01 to 18.0 months was associated with similar to slightly improved outcomes compared with reference (aOR, 1.8; 95\% CI, 1.0-3.0) (data not shown). Results for the pre-XDR-TB subgroup supported the notion that longer intervals were associated with success; effects were statistically significant, with confidence intervals that were wide and overlapping across durations of 15.01 to 24 months (data not shown).
PICO Question 3: Should patients with MDR-TB undergoing continuation-phase treatment be treated for $\geqslant 18$ months after culture conversion or $<18$ months after culture conversion?

Recommendation 3a: In patients with MDR-TB, we suggest a total treatment duration of between 15 and 21 months after culture conversion (conditional recommendations, very low certainty in the evidence).

Recommendation 3 b: In patients with pre-XDR-TB and XDR-TB, we suggest a total treatment duration of between 15 and 24 months after culture conversion (conditional recommendations, very low certainty in the evidence). 
Table 7. Distribution of Patients with Multidrug Resistance by Duration of Treatment from Sputum Culture Conversion to End of Treatment $(N=4,691)$

\begin{tabular}{|c|c|c|c|c|c|}
\hline $\begin{array}{l}\text { Intervals from Sputum } \\
\text { Culture Conversion to } \\
\text { End of Treatment ( } \mathrm{mo} \text { ) }\end{array}$ & $\begin{array}{l}\text { No. of } \\
\text { Patients }\end{array}$ & $\begin{array}{l}\text { Mean Duration of } \\
\text { Intensive Phase } \\
\text { (mo) }\end{array}$ & $\begin{array}{l}\text { Mean Duration to } \\
\text { Sputum Culture } \\
\text { Conversion (mo) }\end{array}$ & $\begin{array}{l}\text { Mean Interval from } \\
\text { Sputum Culture } \\
\text { Conversion to End of } \\
\text { Treatment (mo) }\end{array}$ & $\begin{array}{l}\text { Total Duration of } \\
\text { Treatment (mo) }\end{array}$ \\
\hline $0.1-12.0$ & 396 & 6.7 & 5.7 & 9.3 & 15.0 \\
\hline $12.01-15.0$ & 593 & 6.8 & 3.7 & 13.9 & 17.6 \\
\hline $15.01-18.0$ & 1,235 & 7.3 & 2.1 & 16.9 & 19.0 \\
\hline $18.01-21.0$ & 1,158 & 7.9 & 2.1 & 19.3 & 21.4 \\
\hline $21.01-24.0$ & 893 & 8.8 & 1.9 & 22.4 & 24.4 \\
\hline $24.01-69$ & 416 & 11.9 & 1.9 & 26.8 & 28.7 \\
\hline
\end{tabular}

Harms. Extending treatment longer than necessary can engender additional toxicity and costs to patients and health systems. For this reason, recommendations are for the minimum duration found to have a significant treatment advantage, and different recommendations are made for the MDR only and XDR/pre-XDR subgroups.

Additional considerations. No information on how duration was selected is available from the data. Duration selection may reflect interim response to treatment or may reflect a planned duration that is not conditioned on treatment response; the latter is suggested by the observation that duration was inversely related to time to conversion except in the longest interval. Analyses were adjusted for all possible baseline confounders, relying on PS matching, to minimize the bias introduced. Nevertheless, the possibility of unmeasured confounding by indication, in particular by time-varying characteristics, cannot be ruled out. Such confounding by indication would likely result in an underestimate of the benefit of a longer treatment duration after conversion. No significant difference in outcomes was observed across durations for the XDR-TB subgroup, likely because of small numbers and the aforementioned potential bias.

PICO Question 4-Amoxicillin/Clavulanate: In patients with MDR-TB, are outcomes safely improved when regimens include amoxicillin/clavulanate compared with regimens that do not include amoxicillin/clavulanate?

Recommendation 4: We recommend NOT including amoxicillin-clavulanate in a treatment regimen for patients with MDR-TB, with the exception of when the patient is receiving a carbapenem, wherein the inclusion of clavulanate is necessary (strong recommendation, very low certainty in the evidence). Our recommendation against the use of amoxicillin-clavulanate (except to provide clavulanate when using a carbapenem) in MDR-TB treatment is strong despite the evidence being judged to be of very low certainty because we viewed the increased mortality and decreased likelihood of treatment success associated with the use of this drug as having a notably unfavorable balance of benefits to potential harms. greater precision in selecting and individualizing regimen duration (57).

\section{Drugs and Drug Classes}

These guidelines are intended for settings in which treatment is individualized based on DST results and clinical and epidemiological factors. Individualized treatment regimens should only include drugs to which the patient's isolate has documented or high likelihood of susceptibility. Treatment regimens should favor medications that are associated with improved outcomes, as identified in our PS-matched IPDMA, that limit toxicity and that incorporate patient preferences. The following alphabetically listed drugs and drug classes were considered for inclusion in treatment regimens: amoxicillin/clavulanate, bedaquiline, carbapenem with clavulanic acid, clofazimine, cycloserine, delamanid, ethambutol, ethionamide, fluoroquinolones, injectable agents, linezolid, macrolides, $p$-aminosalicylic acid, and pyrazinamide. For each drug or drug class, the following PICO was addressed: In patients with MDRTB, are outcomes safely improved when regimens include the following individual drugs or drug classes compared with regimens that do not include them?

\section{Amoxicillin/Clavulanate}

Amoxicillin-clavulanate, consisting of the $\beta$-lactam antibiotic amoxicillin and the $\beta$-lactamase inhibitor potassium clavulanate, is considered safe and effective for numerous bacterial infections. Although $M$. tuberculosis has an impenetrable cell wall and produces a $\beta$-lactamase inhibitor (58), amoxicillin-clavulanate has been used to treat TB. It is viewed as a "salvage" agent when few other 
Table 8. Adjusted Estimates of Treatment Success by Duration of Treatment Interval between Sputum Culture Conversion and End of Treatment, All Forms of Multidrug Resistance $(N=4,691)$

\begin{tabular}{|c|c|c|c|c|c|c|}
\hline \multirow[b]{2}{*}{$\begin{array}{l}\text { Interval from Sputum Culture } \\
\text { Conversion to End of Treatment (mo) }\end{array}$} & \multicolumn{2}{|c|}{ No. of Patients } & \multicolumn{4}{|c|}{ Propensity Score-matched Analysis } \\
\hline & $\begin{array}{l}\text { Treatment } \\
\text { Success }\end{array}$ & Total & No. of Pairs & aOR & $95 \% \mathrm{Cl}$ & $\begin{array}{l}\text { Risk Difference } \\
(95 \% \mathrm{Cl})\end{array}$ \\
\hline $0.1-12.0$ & 360 & 396 & 394 & 0.5 & 0.4 to 0.7 & $-0.04(-0.07$ to -0.01$)$ \\
\hline $12.01-15.0$ & 565 & 593 & - & 1.0 & Reference & - \\
\hline $15.01-18.0$ & 1,206 & 1,235 & 1,223 & 2.1 & 1.4 to 3.1 & 0.02 (0.01 to 0.04$)$ \\
\hline $18.01-21.0$ & 1,122 & 1,158 & 1,154 & 1.6 & 1.1 to 2.3 & $0.02(0.00$ to 0.03$)$ \\
\hline $21.01-24.0$ & 858 & 893 & 889 & 1.2 & 0.9 to 1.8 & 0.01 ( -0.01 to 0.02$)$ \\
\hline 24.01-69 & 386 & 416 & 413 & 0.7 & 0.4 to 1.0 & $-0.02(-0.05$ to 0.00$)$ \\
\hline
\end{tabular}

Definition of abbreviations: aOR = adjusted odds ratio; $\mathrm{Cl}=$ confidence interval.

TB drug options remain available because of either drug resistance or intolerance. The data for use of amoxicillin-clavulanate in TB are limited and show mixed results (59-62). In addition, because it is the only source of clavulanate, amoxicillin-clavulanate has been used along with carbapenems. Synergy between meropenem and amoxicillin-clavulanate in XDR TB has been noted $(63,64)$, and the combination has been reported to be efficacious, safe, and tolerable when added to linezolid and other drugs (65-69). See PICO Question 6 for the evaluation of carbapenems with amoxicillin-clavulanate.

Summary of the evidence. Procedures and methodology to assemble and the certainty in the evidence are reported in ApPENDIX A, with evidence profiles for PICO questions reported in ApPENDIX B. Our PS-matched IPDMA showed that patients who received amoxicillinclavulanate were more likely to have been treated with second-line drugs and to be resistant to fluoroquinolones or any second-line injectable (3). They were more likely to have received a later-generation fluoroquinolone $(72 \%)$, capreomycin
(58\%), linezolid (25\%), and bedaquiline (8\%). In adjusted analyses, patients who received amoxicillin-clavulanate were less likely to have treatment success (aOR, 0.6; 95\% CI, 0.5-0.8) and more likely to die (aOR, 1.7; 95\% CI, 1.3-2.1) compared with patients who did not receive amoxicillin-clavulanate.

Benefits. The addition of amoxicillinclavulanate (without coadministration with carbapenems) to a regimen for MDR-TB does not appear to provide benefit.

Harms. Patients who received amoxicillin-clavulanate were less likely to have treatment success and more likely to die than patients who did not receive amoxicillin-clavulanate. Data on adverse effects were not collected systematically across studies in our PS-matched IPDMA or in most trials. A published systematic review identified diarrhea and candidiasis as key adverse effects associated with amoxicillin-clavulanic acid use (70).

Additional considerations. Clavulanic acid is only available as a coformulation with amoxicillin. Therefore, amoxicillin-clavulanate must be given whenever carbapenems are included in an MDR-TB regimen (see PICO Question 6 on carbapenems with clavulanate).
PICO Question 5-Bedaquiline: In patients with MDR-TB, are outcomes safely improved when regimens include bedaquiline compared with regimens that do not include bedaquiline?

Recommendation 5: We recommend including bedaquiline in a regimen for the treatment of patients with MDR-TB (strong recommendation, very low certainty in the evidence). Our recommendation for the use of bedaquiline is strong despite very low certainty in the evidence because we viewed the significant reduction in mortality, improved treatment success, and relatively few adverse effects associated with MDRTB treatment including bedaquiline (compared with no bedaquiline) as having a particularly favorable balance of benefits over harms.
Conclusions. Patients who received amoxicillin-clavulanate were less likely to achieve treatment success and more likely to die than patients who did not receive amoxicillin-clavulanate. These data suggest amoxicillin-clavulanate should not be used in MDR-TB treatment, except to provide clavulanate when using a carbapenem (see PICO Question 6 on carbapenems with clavulanate). Our recommendation against the use of amoxicillin-clavulanate (except to provide clavulanate when using a carbapenem) in MDR-TB treatment is strong despite the evidence being judged to be of very low certainty because we viewed the increased mortality and decreased likelihood of treatment success associated with the use of this drug as having a notably unfavorable balance of benefits to potential harms.

Research needs. The development of a clavulanic acid formulation without amoxicillin, for use in combination with carbapenems, would be helpful and would avoid unnecessary toxicities from amoxicillin as well as adhere to international efforts in promoting antimicrobial stewardship (1).

\section{Bedaquiline}

Bedaquiline, a diarylquinoline, approved by FDA in 2013, is the first drug with a novel mechanism of action against $M$. tuberculosis to have been approved by FDA in $>40$ years $(71,72)$. Bedaquiline is bactericidal to nonreplicating and actively replicating mycobacteria, through ATP synthase inhibition, and has bactericidal and sterilizing activity in the murine model of TB infection (73). No cross-resistance has been found between bedaquiline and the following: isoniazid, rifampin, ethambutol, pyrazinamide, streptomycin, amikacin, or moxifloxacin. There have been a few reports of cross-resistance with clofazimine 
$(74,75)$. Bedaquiline is customarily used as part of combination therapy (minimum four-drug therapy) for adults aged $\geqslant 18$ years with a diagnosis of pulmonary MDRTB when an effective treatment regimen cannot otherwise be provided (e.g., mycobacterial isolates show a complicated drug resistance pattern, drug intolerance, or drug-drug interactions) (76). The recommended dose of bedaquiline for the treatment of pulmonary MDR-TB in adults is $400 \mathrm{mg}$ administered orally once daily for 2 weeks, followed by $200 \mathrm{mg}$ administered orally three times weekly, for an entire treatment duration of 24 weeks $(72,76-78)$. Bedaquiline has recently has been identified as the key drug in assembling an all-oral, injectable-free drug regimen in South Africa (79).

Summary of the evidence. Procedures and methodology to assemble and rank the certainty in the evidence are reported in APPENDIX A, with evidence profiles for PICO questions reported in APPENDIX B. Our PSmatched IPDMA included 411 patients who received bedaquiline-containing regimens, for whom it was assumed that there was no bedaquiline resistance, and 10,932 patients who did not receive bedaquiline (3). Patients who received bedaquiline-containing regimens were more likely to have cavitary disease ( $82 \%$ vs. $62 \%)$, to have been treated with second-line drugs ( $34 \%$ vs. $14 \%$ ), to be infected with an organism resistant to fluoroquinolones ( $57 \%$ vs. $21 \%$ ) or to any second-line injectable (58\% vs. $23 \%$ ), and to have XDR-TB (29\% vs. $13 \%)$. They were also more likely to have received a latergeneration fluoroquinolone (69\% vs. $54 \%$ ), linezolid (64\% vs. $4 \%$ ), or clofazimine $(48 \%$ vs. $4 \%$ ). Treatment success (cure and completion) was slightly greater (70\% vs. $60 \%$; $P=0.001)$ with bedaquiline, whereas failure/relapse (6\% vs. $9 \%)$, death (10\% vs. $15 \%$ ), and loss to follow-up (14\% vs. $16 \%$ ) were less frequent in PS-adjusted analyses.

Benefits. Our PS-matched IPDMA showed that patients treated with bedaquiline-containing regimens were more likely to have treatment success (aOR, 2.0; 95\% CI, 1.4-2.9), less likely to experience failure/relapse, and less likely to die (aOR, 0.4; 95\% CI, 0.3-0.5; absolute risk reduction, $7.2 \%)$. Results were similar when only patients from high-income countries treated with bedaquiline-containing regimens were included and when comparing only patients with XDR-TB who were and were not treated with bedaquiline.
A recent large program-based observational multinational study confirmed a high treatment success rate $(76.9 \%)$ with bedaquiline-containing regimens, with a low proportion $(5.8 \%)$ of interruptions owing to adverse events (80). In our IPDMA, using PS-matched pairs comparing the effects of bedaquiline to those of clofazimine, statistically significant differences for success versus failure/relapse favoring bedaquiline use were observed (aOR, 2.1; 95\% CI, 1.1-4.1) (using >170 PS-matched pairs), but statistically significant findings were not observed in mortality or when restricting analyses to high-income countries. PS-matched pairs analyses comparing the effects of various combinations of drugs used with bedaquiline all noted improved outcomes. When comparisons of effects of bedaquiline and linezolid with those of no bedaquiline or linezolid were performed, combination of bedaquiline and linezolid was associated with an aOR of 2.7 (95\% CI, 1.5-4.9) for success versus failure/relapse and with an aOR of 0.3 (95\% CI, 0.2-0.4) for death versus success/failure/relapse. Similarly, when PS-matched pair analyses comparing the effects of bedaquiline and clofazimine to those of no bedaquiline or clofazimine were performed, combination of bedaquiline and clofazimine was associated with an aOR of 5.0 (95\% CI, 2.4-10.6) for success versus failure/relapse, and with an aOR of 0.3 (95\% CI, 0.2-0.5) for death versus success/failure/relapse. Of note, patients to whom bedaquiline was administered tended to have more cavitary disease or to have preXDR or XDR-TB. Prescription of bedaquiline was associated with greater success and less death than clofazimine, but the greatest success and least mortality was found when bedaquiline was administered together with linezolid or clofazimine.

Finally, a recent retrospective routinecare observational study in South Africa showed that a bedaquiline-containing regimen was associated with significantly lower mortality (128 [12.6\%] deaths among 1,016 patients receiving bedaquiline compared with 4,612 deaths [24.8\%] among 18,601 patients on the standard regimens). Bedaquiline was associated with a reduction in the risk of all-cause mortality for patients with MDR- or RR-TB (hazard ratio [HR], 0.35; 95\% CI, 0.28-0.46) and XDR-TB (HR, 0.26; 95\% CI, 0.18-0.38) compared with standard regimens (50).
Harms. In a review of cases treated with bedaquiline, only 44 of 1,266 (3.5\%) cases with information available discontinued bedaquiline because of adverse events. Only 8 of 875 (0.9\%) discontinued bedaquiline because of QT interval prolongation (two restarted the drug after resolution of the acute episode without further problems) (81).

Additional considerations. When bedaquiline is included in the regimen, most experts obtain ECGs after the initial 2 weeks of therapy and then at monthly intervals to monitor for QT interval prolongation. Serum electrolytes, including calcium, magnesium, and potassium, are also monitored.

The 2013 CDC guidelines on the use of bedaquiline note that there is insufficient evidence to provide guidance on the use of bedaquiline in children but that its use can be considered on a case-by-case basis given the high mortality and limited treatment options for MDR-TB (71). More recently, adolescents $>10$ years old and weight $>34 \mathrm{~kg}$ have been safely treated off-label with the recommended adult dose of bedaquiline (82). The Sentinel Project on Pediatric Drug-Resistant Tuberculosis has recommended that children $\geqslant 12$ years of age and $\geqslant 31 \mathrm{~kg}$ body weight receive bedaquiline at the same dose as adults, and children $\geqslant 6$ years with 16 to $30 \mathrm{~kg}$ body weight could receive half the adult bedaquiline dose for the same indications $(7,17,83)$.

Conclusions. In our PS-matched IPDMA, bedaquiline-containing regimens were more likely to achieve treatment success and to have a lower rate of death than those that did not include bedaquiline. Bedaquiline should be included in a regimen to achieve a total of five effective drugs for the treatment of patients with MDR-TB. Our recommendation for the use of bedaquiline is strong despite very low certainty in the evidence because we viewed the significant reduction in mortality, improved treatment success, and relatively few adverse effects associated with MDR-TB treatment including bedaquiline (compared with no bedaquiline) as having a particularly favorable balance of benefits over harms.

Research needs. Further research is needed to elucidate the potential synergy of bedaquiline with other agents. Studies underway are evaluating the use of bedaquiline together with linezolid, clofazimine, or nitroimidazoles 
(i.e., pretomanid and delamanid). Research is also needed on the safety, tolerability, and efficacy of bedaquiline-based shorter-course regimens as well as on the use of bedaquiline for durations $>24$ weeks, an approach currently considered when effective treatment cannot otherwise be provided $(71,84)$. Finally, research is urgently needed on the risk factors and any interventions (e.g., the selection of companion drugs) that influence acquisition of bedaquiline resistance.

\section{Carbapenems with Clavulanic Acid}

The combination of carbapenems and clavulanate, a $\beta$-lactamase inhibitor, has been shown to have in vitro bactericidal activity $(65,85,86)$. As clavulanate is not available by itself, the combination drug amoxicillin-clavulanate must be given with the carbapenems. Carbapenems have been used primarily for MDR and $\mathrm{XDR} \mathrm{TB}$, and a recent systematic review found that carbapenems are safe and likely to be effective (65). Carbapenem drugs have been recently included in WHO guidelines for the treatment of DR-TB (23).

Summary of the evidence. Procedures and methodology to assemble and rank the certainty in the evidence are reported in Appendix A, with evidence profiles for PICO questions reported in Appendix B. Our PS-matched IPDMA compared death, treatment success, and culture conversion among 169 individuals who received carbapenems to 9,535 individuals from centers that did not use any of the drugs previously classified by WHO as "Group 5" drugs (3). In addition, we considered adverse events reported in a recent review (65), IPDMA, and systematic review that summarize the available evidence on carbapenems from five primary studies (all

PICO Question 6-Carbapenems with clavulanic acid: In patients with MDR$\mathrm{TB}$, are outcomes safely improved when regimens include carbapenems with clavulanic acid compared with regimens that do not include them?

Recommendation 6: We suggest including a carbapenem (always to be used with amoxicillin-clavulanic acid) in a regimen for treatment of patients with MDR-TB (conditional recommendation, very low certainty in the evidence). observational studies) (23, 65-67, 87-92). The reviewed evidence showed that there was concomitant use of linezolid and bedaquiline in large proportions of the patient population receiving carbapenems, suggestive of confounding, which was considered in the review of the efficacy results.

Benefits. In our PS-matched IPDMA, inclusion of carbapenems in the treatment regimen had no effect on the risk of death (aOR, 1.0; 95\% CI, 0.5-1.7) or culture conversion (aOR, 2.3; 95\% CI, 0.8-6.9). However, treatment success was more likely among patients treated with regimens including a carbapenem compared with those treated with regimens not including a carbapenem (aOR, 4.0; 95\% CI, 1.7-9.1).

Harms. In our meta-analysis, the rate of treatment discontinuation due to an adverse event was lower among patients treated with regimens including a carbapenem than those treated with regimens not including a carbapenem, although this difference was not statistically significant (relative risk, 0.82; 95\% CI, $0.23-2.94)$. In the published literature, treatment discontinuation due to adverse events has been reported in 0 to $3 \%$ of patients, with minor adverse events occurring in $5 \%$ to $6 \%$ of patients (65).

Additional considerations. Of note, clavulanic acid is only available as a coformulation with amoxicillin. Therefore, amoxicillin-clavulanate must be given to achieve a dose of $125 \mathrm{mg}$ of the clavulanate with each dose of carbapenem daily, whenever carbapenems are included in an MDR-TB regimen. DST is currently not available for carbapenems. When used, imipenem-cilastatin/clavulanate or meropenem/clavulanate have been administered intravenously in a hospital setting, with multiple injections required daily (67). There is no clear evidence of whether imipenem-cilastatin or meropenem is more efficacious (66). Ertapenem belongs to the same family $(65,93)$; because of its longer half-life and the possibility to administer it once a day intramuscularly or intravenously, ertapenem may be useful when a patient treated with meropenem or imipenem-cilastatin intravenously during hospitalization is discharged and needs to continue carbapenem-based treatment outpatient (92). Long-term intravenous administration requires long-term venous access through an indwelling catheter, which carries risks of infection, thrombosis, and thromboembolism. Data on the use of a carbapenem combined with amoxicillin-clavulanate in children are lacking-only two adolescents are included in one review (65). However, efficacy has been shown in adults, and both carbapenems and amoxicillinclavulanate have been used safely in children for other purposes (although there are no long-term treatment safety data in young children); therefore, this combination could be used in children with MDR-TB where there is no other option to build an effective regimen.

Conclusions. In our PS-matched IPDMA, inclusion of carbapenems with clavulanic acid was associated with an increase in treatment success when compared with the control group not receiving carbapenems with clavulanic acid. Carbapenems with clavulanic acid can be included in a regimen to achieve a total of five effective drugs for the treatment of patients with MDR-TB.

Research needs. Randomized, controlled clinical trials that confirm the role of carbapenems used for different durations of time within different regimens are needed. A comparative evaluation of the safety, tolerability, and efficacy of the different agents is also necessary. Because of the high cost of these drugs, economic analyses will also be useful (65). The development of oral formulations of carbapenems is underway, which, if proven safe and effective, would enhance feasibility of using these agents significantly. Additional advances in rapid DST for carbapenems would enhance potential scale-up of use for this class of drugs.

PICO Question 7-Clofazimine: In patients with MDR-TB, are outcomes safely improved when regimens include clofazimine compared with regimens that do not include clofazimine?

Recommendation 7: We suggest including clofazimine in a regimen for treatment of patients with MDR-TB (conditional recommendation, very low certainty in the evidence). 


\section{Clofazimine}

Clofazimine, a fat-soluble riminophenazine dye, has shown in vitro and in vivo activity as a sterilizing drug to treat MDR-TB (94-96).

Clofazimine has been used as a leprosy drug and was first developed in the 1950s because of in vitro and in vivo activity against M. tuberculosis $(94,95,97)$. Although the exact mechanism of action is not known, it is a prodrug that appears to have both antimycobacterial and antiinflammatory properties (98). The published clinical evidence on the safety and efficacy of clofazimine for treatment of TB is modest (99). However, interest in the drug has increased since WHO endorsed the new shorter-course regimen $(23,100)$, which includes clofazimine (101-108).

Summary of the evidence. Procedures and methodology to assemble and rank the certainty in the evidence are reported in APPENDIX A, with evidence profiles for PICO questions reported in APPENDIX B. Our PSmatched IPDMA compared death, treatment success, and culture conversion among 639 individuals who received clofazimine to 7,398 individuals from centers that did not use any of the drugs previously classified by WHO as "Group 5" drugs (3). In addition, we considered adverse events reported in a published systematic review of nine observational studies (six MDR-TB and three XDR-TB) including 599 patients treated with clofazimine (427 from a single cohort study from Bangladesh) (95) and two studies published after the systematic review. They include a trial at six specialty hospitals in China that randomized 105 patients to 21 months of treatment with an optimized background regimen with or without clofazimine (100 mg/d) (94) and a retrospective cohort study from Brazil that compared outcomes in patients with MDR-TB treated with clofazimine (100 mg/d)-containing regimens versus pyrazinamide-containing regimens (109).

Benefits. Our PS-matched IPDMA showed that treatment success was more likely with regimens containing clofazimine compared with regimens that did not (aOR, 1.5; 95\% CI, 1.1-2.1). Addition of clofazimine was associated with an aOR of 0.8 (95\% CI, 0.6-1.0) for the outcome of death and with an aOR of 1.1 (95\% CI, 0.6-1.8) for culture conversion within 6 months. As noted in PICO Question 5 on bedaquiline, when PSmatched pair analyses were performed comparing the effects of bedaquiline and clofazimine to those of no bedaquiline or clofazimine, the combination of bedaquiline and clofazimine was associated with an aOR of 5.0 (95\% CI, 2.4-10.6) for success versus failure/relapse and with an aOR of 0.3 (95\% CI, $0.2-0.5$ ) for death versus success/failure/relapse.

Harms. In our PS-matched IPDMA, 2 of $81(2.5 \%)$ patients treated with regimens including clofazimine had treatment discontinued because of adverse events. Brownish skin pigmentation has been observed in $75 \%$ to $100 \%$, ichthyosis in $8 \%$ to $20 \%$, gastrointestinal intolerance in $40 \%$ to $50 \%$, and neurological disturbances in up to $13 \%$ of patients $(94,95,109)$. Clofazimine was judged to have small to moderate desirable effects (on treatment success, mortality, and culture conversions) and small undesirable effects (low risk of serious adverse events requiring treatment discontinuation). However, there is important uncertainty about how patients value skin discoloration. Some patients perceive skin discoloration as significant, which may become a key factor in the acceptability of this drug in some settings. Recent publications have noted the potential QT interval-prolonging effects of clofazimine when used in combination with bedaquiline and delamanid (110-112).

Additional considerations. Access is a limitation to expanded use of clofazimine for treatment of MDR-TB, especially in the United States and Europe, where clofazimine lacks a TB indication. In the United States, clofazimine is currently only available under an investigational new drug protocol administered by FDA, which can be a burdensome procedure. Significant improvements in the mechanisms for accessing clofazimine are necessary for more expanded use of this drug. Quality-assured clofazimine is available through the Global Drug Facility, although currently in limited quantities. Although our PS-matched IPDMA had limited pediatric data, the efficacy in children is believed by experts to be the same as in adults. A recent IPDMA in children with MDR-TB did not show a benefit using clofazimine, which might be related to selection of cases and very small numbers (23 of 641) receiving clofazimine (113). Moreover, children are included in the WHO shortercourse regimen for MDR-TB treatment, which includes clofazimine, albeit on the basis of limited data. Dosing clofazimine in children is challenging because of the lack of childfriendly formulations and lack of pharmacokinetic data. The currently recommended dose for clofazimine in children varies from 2 to $5 \mathrm{mg} / \mathrm{kg}$ daily. Skin discoloration is common; some improve during treatment and others resolve rapidly after discontinuation of treatment. Ichthyosis is less common and improves with intensive efforts at applying lubricants during treatment. As noted, QT interval prolongation may occur and is especially important to monitor by ECG if given together with other QT interval-prolonging medication $(68,83$, $110-112,114,115)$. Clofazimine may have cross-resistance with bedaquiline, which may need to be considered when building a regimen for MDR-TB (75).

Conclusions. In our PS-matched IPDMA, inclusion of clofazimine was associated with increase in treatment success when compared with the control group not receiving clofazimine.

Clofazimine can be included in a regimen to achieve a total of five effective drugs for the treatment of patients with MDR-TB.

Research needs. The value of using loading doses and the optimal dosing for clofazimine requires additional research. Pharmacokinetic data in adults and children are needed.

\section{Cycloserine}

Cycloserine is an oral bacteriostatic drug that has been part of the backbone regimen for MDR-TB treatment in the past. Cycloserine is a broad-spectrum antibiotic that inhibits cell wall synthesis $(116,117)$. Terizidone is a structural analog that is a combination of two cycloserine molecules; it appears to be used interchangeably by experts, although it is currently not available in the United States (118). Although some advantages of cycloserine include the absence of crossresistance to other drugs and reasonable gastrointestinal tolerability, a significant drawback relates to psychological side effects, which occasionally necessitate

discontinuation of the drug (16).

Summary of the evidence. Previous studies showed an increase of the likelihood of treatment success when cycloserine was included in the MDR-TB regimen (marginally statistically significant) (96). Procedures and methodology to assemble and rank the certainty in the evidence are reported in APPENDIX A, with evidence profiles for PICO questions reported in ApPENDIX B.

Benefits. Our PS-matched IPDMA showed that inclusion of cycloserine was associated with an increase in treatment success (aOR, 1.5; 95\% CI, 1.4-1.7) and a decrease in 
PICO Question 8-Cycloserine: In patients with MDR-TB, are outcomes safely improved when regimens include cycloserine compared with regimens that do not include cycloserine?

Recommendation 8: We suggest including cycloserine in a regimen for treatment of patients with MDR-TB (conditional recommendation, very low certainty in the evidence).

mortality (aOR, 0.6; 95\% CI, 0.5-0.6) when compared with the control group. A published IPDMA of children with successful treatment of MDR-TB showed that cycloserine as a single drug had an aOR of 1.7 (95\% CI, 0.9-3.0; 641 children with MDR-TB, of whom 356 [56\%] received cycloserine/terizidone) (113). Limited pharmacokinetic data are available on cycloserine (or terizidone) in children, but a recent study of 25 children (only 5 to $<12$ yr of age) receiving a median of $14.3 \mathrm{mg} / \mathrm{kg}$ (range, $10.0-18.0 \mathrm{mg} / \mathrm{kg}$ ) showed that the maximum concentration of the drug achieved in the plasma after dose administration was similar to the adult target maximum concentration of 20 to $35 \mathrm{mg} / \mathrm{L}$ (119). The recommended dose for children of 10 to 20 $\mathrm{mg} / \mathrm{kg} / \mathrm{d}$ seems adequate. An advantage of cycloserine in children, as in adults, with miliary and/or central nervous system (CNS) TB is that it penetrates the CNS well. Cycloserine may interfere with the pharmacokinetics and absorption of isoniazid and ethionamide/prothionamide; therefore, it may be advisable to give it separately from these drugs if used together in the same regimen (117).

Harms. Cycloserine has CNS adverse effects, which are reported to occur in about $20 \%$ to $30 \%$ of adults (120). A meta-analysis of published articles identified adverse events in 201 of 2,164 patients across all studies (118). An average weighted proportion of patients receiving cycloserine who discontinued treatment owing to adverse effect pooled across the studies was $9.1 \%$ (95\% CI, 6.4-11.7\%). An average weighted proportion of patients with psychiatric adverse effects was 5.7\% (95\% CI, 3.7-7.6\%) (118). Limited data exist on cycloserine adverse effects in children. In older pediatric studies, no adverse effects were reported with the use of cycloserine (121-123). In a systematic review of outcomes of MDR-TB in children, 6 of 182 (3.3\%) children had adverse effects attributed to cycloserine, which included depression, anxiety, hallucinations, transitory psychosis, and blurred vision (124).

Additional considerations. Lowerthan-recommended serum cycloserine concentrations and delayed absorption have been reported (125). Some experts obtain peak concentrations within the first 1 to 2 weeks of therapy and continue to monitor serially. Because of specific technical challenges related to cycloserine DST, poor accuracy of testing in liquid media, and poor intrinsic reproducibility of results, few laboratories perform DST for cycloserine (16).

Conclusions. In our PS-matched IPDMA, inclusion of cycloserine was associated with a decrease in mortality and increase in treatment success when compared with the control group not receiving cycloserine. Cycloserine can be included in a regimen to achieve a total of five effective drugs for the treatment of patients with MDR-TB.

Research needs. Studies are needed to determine the effect of cycloserine on the absorption of isoniazid and ethionamide.

\section{Delamanid}

Delamanid is a nitro-dihydro-imidazooxazole derivative, which was approved for the treatment of MDR-TB by the European Medicines Agency in 2013 but has not yet received FDA approval. In 2014, WHO issued interim policy guidance on the use of delamanid for the treatment of MDR-TB on the basis of phase $2 \mathrm{~b}$ clinical trial data. The interim policy guidance stated that "delamanid may be added to an MDR-TB regimen in adult patients with pulmonary $\mathrm{TB}$ conditional upon: i) careful selection of patients likely to benefit; ii) patient informed consent; iii) adherence to $\mathrm{WHO}$ recommendations in designing a longer MDR-TB regimen; iv) close monitoring of clinical treatment response; and v) active $\mathrm{TB}$ drug-safety monitoring and management (aDSM)" (126). WHO issued an updated position statement on the use of delamanid for MDR-TB in 2018 on the basis of final results from the phase 3 randomized controlled trial, Trial 213 (127).

Summary of the evidence. Delamanid data were not available as part of the PS-matched IPDMA completed for this guideline development process; however, the 2019 WHO Consolidated Guidelines on Drug-Resistant Tuberculosis Treatment, wherein individual-level data on delamanid were attained for analyses, provides practice guidance on the use of delamanid for treatment of MDR-TB $(7,128)$. Several recent reports, clinical trials, and cohort studies report on delamanid-containing regimens achieving success rates of $77 \%$ to $84 \%$, in absence of major adverse events (128-131). In the United States, through a compassionate use program, delamanid has recently been successfully accessed and used in the care of patients with MDR-TB (132). Regarding use of delamanid in children, only a small number of children ( $>6 \mathrm{yr}$ of age) have had documented access to the drug, also by compassionate use (133, 134). On the basis of review of the data available in the IPDMA conducted by WHO, delamanid has been included in "Group C" in their guidelines, corresponding to drugs that can be added "to complete the regimen and when medicines from Groups A and B cannot be used." WHO has recommended that "delamanid may be included in the treatment of MDR/RR-TB patients aged 3 years or more on longer regimens" $(7,135)$. The guideline writing committee concurs with the updated 2019 WHO guidance (7). As noted for other drugs discussed in this practice guideline, QT interval
PICO Question 9-Delamanid: In patients with MDR-TB, are outcomes safely improved when regimens include delamanid compared with regimens that do not include delamanid?

Recommendation 9: The guideline panel was unable to make a clinical recommendation for or against delamanid because of the absence of data in the PSmatched IPDMA conducted for this practice guideline. We make a research recommendation for the conduct of randomized clinical trials and cohort studies evaluating the efficacy, safety, and tolerability of delamanid in combination with other oral agents. Until additional data are available, the guideline panel concurs with the conditional recommendation of the 2019 WHO Consolidated Guidelines on DrugResistant Tuberculosis Treatment that delamanid may be included in the treatment of patients with MDR/RR-TB aged $\geqslant 3$ years on longer regimens (7). 
prolongation may occur with delamanid use, and routine ECG monitoring is recommended. A recent evaluation of the cardiac safety of delamanid and bedaquiline given together as part of multidrug therapy for MDR-TB concluded that the combined effect on the corrected QT interval (QTc) using the Fridericia formula (QTcF) is clinically modest and no more than additive (ClinicalTrials.gov Identifier: NCT02583048), with mean change in QTcF from baseline at 11.9 milliseconds $(95.1 \%$ CI, 7.4-16.5 ms) in the bedaquiline arm, 8.6 milliseconds (95.1\% CI, 4.0-13.2 ms) in the delamanid arm, and 20.7 milliseconds (95.1\% CI, 16.1-25.4 ms) in the combined bedaquiline and delamanid arm (136).

Conclusions. The guideline panel was unable to make a recommendation for or against delamanid because of the absence of data in the IPDMA conducted for this practice guideline. In 2018, WHO updated the IPDMA with additional data and recommended delamanid be included in the third tier of drugs, Group C, and for the drug to be used in the treatment of patients with MDR/RR-TB aged $\geqslant 3$ years on longer regimens. The guideline writing committee agrees with the updated 2019 WHO Consolidated Guidelines on Drug-Resistant Tuberculosis Treatment that delamanid may be included in the treatment of patients with MDR/RR-TB aged $\geqslant 3$ years on longer regimens (7). We make a research recommendation for the conduct of randomized clinical trials and cohort studies evaluating the efficacy, safety, and tolerability of all-oral, shortened regimens inclusive of delamanid in combination with other oral agents.

Research needs. The companion drugs with which to combine delamanid for

PICO Question 10-Ethambutol: In patients with MDR-TB, are outcomes safely improved when regimens include ethambutol compared with regimens that do not include ethambutol?

Recommendation 10: We suggest including ethambutol in a regimen for treatment of patients with MDR-TB only when more effective drugs cannot be assembled to achieve a total of five effective drugs in the regimen (conditional recommendation, very low certainty in the evidence). optimal efficacy, safety, and tolerability remain uncertain. The conduct of randomized clinical trials and cohort studies evaluating all-oral, shortened regimens, inclusive of delamanid and in combination with other oral agents are urgently needed. The endTB trial is a phase 3 multicountry randomized clinical trial evaluating the efficacy and safety of five new, all-oral, shortened regimens including combinations of bedaquiline and delamanid

(ClinicalTrials.gov Identifier: NCT02754765). Recent cohort data are also emerging on the use of delamanid and bedaquiline in combination, which to date has been generally reserved for severe cases with extensive resistance and when other options are not feasible (137-140). The Nix-TB (A Phase 3 Study Assessing the Safety and Efficacy of Bedaquiline Plus PA-824 Plus Linezolid in Subjects with Drug-Resistant Pulmonary Tuberculosis) trial (ClinicalTrials.gov Identifier: NCT02333799) evaluated an all-oral 6-month regimen comprising bedaquiline, pretomanid (member of the nitroimidazooxazines class of compounds), and linezolid for the treatment of either XDR-TB or treatment-intolerant or nonresponsive MDR-TB. FDA granted priority review of the new drug application for pretomanid in March 2019, the Antimicrobial Drugs Advisory Committee discussed pretomanid in June 2019, and in August 2019 FDA approved pretomanid in combination with bedaquiline and linezolid for the treatment of a specific limited population of adults with pulmonary XDR$\mathrm{TB}$ or treatment-intolerant or nonresponsive MDR-TB (2, 141). Given FDA approval, additional trials that include diverse patient populations will be essential to understand optimal use of the regimen, in addition to head-to-head comparisons of pretomanid with delamanid to determine if these drugs can be used interchangeably.

\section{Ethambutol}

Ethambutol is an ethylenediamine that inhibits arabinosyl transferases, which contribute to $M$. tuberculosis cell wall synthesis (142). The drug is included in standard regimens for treatment of drugsusceptible TB and commonly used in regimens for $\operatorname{MDR}-\mathrm{TB}(11,16,23)$. The published evidence available on its safety and efficacy is modest, and its use as part of the first-line drug-susceptible TB regimen rests largely on its ability to prevent the emergence of resistance to the other drugs in the regimen rather than on its own sterilizing activity (143). Ethambutol has been demonstrated to have modest sterilizing activity as part of combination regimens when used against ethambutolsusceptible isolates for treatment of drugsusceptible TB. The drug is not effective when used for ethambutol-resistant isolates, and such use is not recommended $(54,96)$.

Summary of the evidence. Procedures and methodology to assemble and rank the certainty in the evidence are reported in APPENDIX A, with evidence profiles for PICO questions reported in APPENDIX B. Our PS-matched IPDMA compared outcomes among 3,002 individuals with isolates susceptible to ethambutol who received the drug to 667 individuals with isolates susceptible to ethambutol who did not receive the drug (3). Receipt of ethambutol was associated with an aOR of 0.9 (95\% CI, 0.7-1.1) for the outcome of treatment success; receipt of ethambutol was associated with an aOR of 1.0 (95\% CI, 0.9-1.2) for the outcome of death. However, the groups were not balanced with regard to other effective drugs; in particular, the patients not receiving ethambutol were more likely to receive linezolid. Thus, the ability to detect an effect of ethambutol might have been obscured. In a previous IPDMA, before linezolid and bedaquiline use were common, administration of ethambutol among patients with susceptible isolates was associated with an aOR of 1.7 (95\% CI, 1.2-2.4) for cure/complete versus failure/relapse and an aOR of 1.6 (95\% CI, 1.1-2.4) for cure/complete versus failure/relapse/death, compared with patients receiving ethambutol whose isolates were resistant in vitro (144).

Benefits. Our PS-matched IPDMA showed that treatment success was not significantly more likely with regimens containing ethambutol. However, a number of earlier studies indicate that ethambutol is associated with increased success when used to treat patients whose isolates are susceptible to the drug (143). Moreover, these earlier studies determined that the effectiveness of ethambutol is directly related to dose, and that a dose of $25 \mathrm{mg} / \mathrm{kg}$ was more effective than a dose of $15 \mathrm{mg} / \mathrm{kg}$ (143). Dosages were not available for analysis in our PS-matched IPDMA. Finally, our PS-matched IPDMA did not address a key attribute of ethambutol, the prevention of the emergence of resistance, which is a substantial concern among patients with MDR-TB (145). 
Harms. In a recent review of the tolerability of TB drugs, ethambutol was associated with serious adverse events in 6 of $1,325(0.5 \%)$ patients (23). This is consistent with previous reports of ethambutol toxicity, in which optic neuropathy (including optic neuritis and retrobulbar neuritis) was attributed to ethambutol, manifesting as symptoms of decreased visual acuity, scotomata, color blindness, or visual defects. These toxic effects are dose dependent and, although serious, are generally reversible if recognized promptly and the drug is discontinued or the dose reduced.

Additional considerations. When ethambutol is used, some experts recommend using the higher dose of $25 \mathrm{mg} / \mathrm{kg}$, which is associated with increased efficacy but also slightly greater ocular toxicity. Other experts recommend using 15 to $20 \mathrm{mg} / \mathrm{kg}$, counting the drug predominantly as providing protection against the acquisition of additional resistance. All patients receiving ethambutol as part of an MDR-TB treatment regimen should be monitored monthly for signs of ocular toxicity, particularly visual impairment, and if this is detected, ethambutol should be discontinued. If optic neuritis occurs in patients taking both ethambutol and linezolid, both drugs must be stopped. Many patients may be rechallenged successfully with linezolid once vision normalizes, but rechallenge with ethambutol is not recommended. The efficacy of ethambutol is not expected to be different in children compared with adults. A dose of $25 \mathrm{mg} / \mathrm{kg}$ is recommended by experts for children with MDR-TB. Two reviews on safety of ethambutol relating to vision have shown low risk of ocular toxicity $(143,146)$. However, other drugs, such as linezolid, may also cause optic neuritis, and this should be taken into consideration when treating MDR-TB in children, especially as screening for visual loss is difficult in young children.

Conclusions. We suggest including ethambutol in a regimen for treatment of patients with MDR-TB only when more effective drugs cannot be used to achieve a total of five effective drugs in the regimen (conditional recommendation, very low certainty in the evidence). The committee was divided on the value of recommending ethambutol, given the drug was not associated with any benefit in terms of treatment success or mortality. Concerns about the comparability of IPDMA patient groups treated with and without ethambutol were noted. Unlike ethionamide/prothionamide, where there are substantial undesirable effects, ethambutol had small undesirable effects (low risk of serious adverse events requiring treatment discontinuation). Finally, our PS-matched IPDMA did not address the prevention of emergence of resistance-a recognized attribute of ethambutol in the treatment of drug-susceptible TB-although the applicability of this attribute to MDR-TB regimens is unknown. Overall, the committee suggested that ethambutol can be used only when more effective drugs cannot be assembled to achieve the necessary five effective drugs in the regimen.

Research needs. More evidence is needed on the beneficial role that ethambutol may play in preventing emergence of drug resistance in MDR-TB, including to newer oral drugs. More research is also needed to improve on the reliability of DST for ethambutol in different settings.

\section{Ethionamide and Prothionamide}

Ethionamide and prothionamide are derivatives of isonicotinic acid, somewhat similar in structure to isoniazid. Ethionamide is a prodrug and requires activation, after which it inhibits mycobacterial fatty acid synthesis that is necessary for cell wall synthesis and repair. Clinical studies indicate efficacy of these drugs, and they have been included in regimens for treatment of MDR$\mathrm{TB}$ and for treatment of TB meningitis in adults and children $(16,23,147,148)$.

Summary of evidence. Procedures and methodology to assemble and rank the certainty in the evidence are reported in APPENDIX A, with evidence profiles for PICO questions reported in APPENDIX B. Our PS-matched IPDMA showed that ethionamide/prothionamide were not associated with any benefit, even if susceptible by phenotypic DST (3). Previous studies have shown, however, an increase in the likelihood of treatment success if ethionamide was included in the MDR-TB regimen $(23,149)$. These different results can be explained by the inclusion of newer and more effective drugs, improving outcomes in the comparator groups, potentially biasing the observed effect against ethionamide; fewer patients in the ethionamide/prothionamide group, compared with control subjects, received newer-generation fluoroquinolone ( $51 \%$ vs. $76 \%$ ), amikacin ( $17 \%$ vs. $35 \%)$, and linezolid (5\% vs. $18 \%$ ), and more patient in the ethionamide/prothionamide groups received kanamycin (51\% vs. $12 \%$ ) and capreomycin (27\% vs. $16 \%)$.

Benefits. No benefits were identified for inclusion of ethionamide/prothionamide in regimens, even if susceptible by phenotypic DST. A pediatric IPDMA of MDR-TB cases also did not show benefit using ethionamide/ prothionamide; however, the majority of children (590 of 641) in this study did receive ethionamide or prothionamide, potentially limiting the ability to discern a benefit (113).

Harms. The potential of ethionamide to cause adverse events can also limit its tolerability (120). A review of studies comparing a regimen containing ethionamide or prothionamide (all studies before 1970) showed that adverse effects leading to discontinuation of treatment were equally frequent with ethionamide $(11.3 \%$; range $6-42 \%)$ and prothionamide $(11.9 \%$; range 6-40\%). Adverse effects, when reported, included abnormal liver function tests, gastrointestinal intolerance, endocrine dysfunction, and hypothyroidism, the latter occasionally requiring treatment with thyroxine (147). Hypothyroidism is a common adverse effect of ethionamide (experienced by approximately 20\%) (150, $151)$ and is particularly noted if used with p-aminosalicylic acid and in HIV-infected children (152). Gastrointestinal disturbance is common but usually resolve within the first 2 weeks of treatment in children.

Additional considerations. When ethionamide/prothionamide is included in a regimen for which five other effective drugs cannot be assembled, experts recommend dose escalation (drug ramping) at time of treatment initiation, as well as monitoring of thyroid-stimulating hormone for evidence of hypothyroidism requiring replacement (16). Of note, in the presence of inhA mutation, many isolates will show

\section{PICO Question 11-Ethionamide/} prothionamide: In patients with MDR$\mathrm{TB}$, are outcomes safely improved when regimens include ethionamide/ prothionamide compared with regimens that do not include ethionamide/prothionamide?

Recommendation 11: We suggest NOT including ethionamide/ prothionamide in a treatment regimen for patients with MDR-TB if newer and more effective drugs are available to construct a regimen with at least five effective drugs (conditional recommendation, very low certainty in the evidence). 
cross-resistance between isoniazid and ethionamide/prothionamide (153).

Conclusions. When the individualized treatment regimen for patients with MDR-TB contains newer-generation, moreeffective drugs, the addition of ethionamide/prothionamide does not appear to provide benefit.

Research needs. Research efforts are underway to identify potential boosters of potency for ethionamide, which, if successful when coadministered, may result in improved therapeutic index and overall better risk-benefit ratio for use (154).

\section{Fluoroquinolones: Levofloxacin, Moxifloxacin, Ciprofloxacin, and Ofloxacin}

The fluoroquinolones are a family of chemically related drugs characterized by a common core dual-ring structure (155). Ofloxacin, then levofloxacin, then moxifloxacin sequentially improved on earlier generation's spectrum of activity, including mycobacteria, and their antimycobacterial action increased as evidenced by lower minimum inhibitory concentrations (MICs) and increasing success in clinical use $(156,157)$. Physicians began using these drugs to treat MDR-TB on the basis of in vitro data, with subsequent case series and observational studies showing efficacy (158), although none of the fluoroquinolones are currently indicated by regulatory authorities for the treatment of TB. In general, these drugs are well absorbed orally, have favorable pharmacological profiles for once-daily dosing, are generally well tolerated, and now are available in generic formulations (155).

Summary of the evidence. Procedures and methodology to assemble and rank the certainty in the evidence are reported in APPENDIX A, with evidence profiles for PICO questions reported in APPENDIX B. In our PSmatched IPDMA, ofloxacin was used most commonly $(n=4,020)$, followed by levofloxacin $(n=3,872)$, moxifloxacin $(n=2,132)$, and ciprofloxacin $(n=431) ; 734$ patients did not receive a fluoroquinolone, the comparison group for subsequent analyses, and 828 patients received two or more quinolones and were excluded (3). The groups were similar in age, sex, proportion AFB smear positive, proportion with cavitary disease on chest radiograph, and prior treatment with first-line drugs. However, the no-quinolone group had substantially more HIV coinfection (44\% vs. $13-28 \%$; proportion with HIV coinfection across different quinolones), more past treatment with secondline drugs (52\% vs. $8-25 \%)$, more quinolone resistance ( $86 \%$ vs. $6-33 \%)$, more second line injectables resistance (74\% vs.12-34\%), and more XDR TB (73\% vs. $4-19 \%)$. In terms of treatment, the no-fluoroquinolone group received less amikacin (7\% vs. 18-43\%) and kanamycin (13\% vs, $26-65 \%)$ and more capreomycin (66\% vs. $6-34 \%)$. Therefore, the median number of effective drugs (intensive phase) was lower in the no-fluoroquinolone group (2.1) than in the other groups (3.3-4.0). Thus, treatment success was lower in the nofluoroquinolone group (35\% vs. $55-68 \%$ ) and failure/relapse was higher than but not greatly different from the others ( $13 \%$ vs. $2-10 \%)$; mortality, however, was much higher ( $40 \%$ vs. 11-15\%).

Benefits. In our PS-matched IPDMA, among patients with susceptible isolates, levofloxacin-containing regimens compared with no quinolone were associated with significantly more treatment successes (aOR, 4.2; 95\% CI, 3.3-5.4) and significantly fewer deaths (aOR, 0.6; 95\% CI, 0.5-0.7).

Moxifloxacin, compared with no quinolone, was also associated with significantly more treatment successes (aOR, 3.8; 95\% CI, 2.8-5.2) and significantly fewer deaths (aOR, 0.5; 95\% CI, 0.4-0.6). In the subgroup with resistance to an injectable drug(s), levofloxacin or moxifloxacin were associated with a significant improvement in treatment success (aOR, 1.8; 95\% CI, 1.2-2.8) and reduction in death (aOR, 0.6; 95\% CI, 0.4-0.8), although the corresponding adjusted risk differences were not statistically significant. In pairwise comparisons, both levofloxacin and moxifloxacin were associated with significantly better treatment outcomes than ofloxacin. The aORs of death were lower for the two later-generation quinolones when compared with ofloxacin (levofloxacin: aOR, 0.8; 95\% CI, 0.6-0.9; moxifloxacin: aOR, 0.8; 95\% CI, 0.6-1.0) (data not shown). Ofloxacin and ciprofloxacin are considered inferior quinolones against $M$. tuberculosis (144, 149, 159, 160). Levofloxacin and moxifloxacin did not differ significantly from each other.

In a recent IPDMA describing treatment outcomes in children treated for MDR-TB (113), new and repurposed TB drugs, including late-generation fluoroquinolones, were not used enough to adequately evaluate efficacy, but most experts and emerging evidence suggest that the efficacy of fluoroquinolones noted in adults should be similar in children $(83,161)$.

Harms. In our PS-matched IPDMA, grade 3 adverse events were recorded systematically in a subset of 1,962 patients from a cohort study of MDR-TB at 27 sites in nine countries. Permanent discontinuation of fluoroquinolones due to adverse events was uncommon. Among 150 patients treated with levofloxacin, the drug was stopped permanently because of adverse events in 6 (4.0\%). Among 398 patients treated with moxifloxacin, the drug was stopped permanently in $14(3.5 \%)$ patients. Among 1,167 patients treated with ofloxacin, the drug was stopped permanently in $56(4.8 \%)$ patients. An analysis of 56 clinical trials comparing quinolones against placebo or against other antimicrobial agents found generally similar adverse event profiles (3). Seven studies reported more frequent adverse events and six studies reported fewer adverse events in fluoroquinolone-treated patients. The most frequent adverse effects reported are those of the gastrointestinal tract in $3 \%$ to $17 \%$ and CNS in $0.9 \%$ to $11 \%$ of patients (155, 162). Allergic and other hypersensitivity reactions and other skin reactions occur in $0.5 \%$ to $2.8 \%$ of patients. Other adverse effects that occur in $>1 \%$ of patients are cardiac (QT interval prolongation) and endocrine (hypoglycemia). Recently, FDA strengthened warnings in the prescribing information for the entire class of fluoroquinolones on risks of severe hypoglycemia, certain mental health side effects, and tendonitis, as well as risks of ruptures or tears in the aorta (163). Safety concerns persist for long-term pediatric use of fluoroquinolones, especially regarding arthropathy. However, several long-term prospective and retrospective studies in children have confirmed that severe adverse effects with the fluoroquinolones are rare, including musculoskeletal, neurological, and QT interval prolongation adverse effects (164-166).

Additional considerations. As latergeneration fluoroquinolones have become generic, their cost has decreased greatly and their use has expanded to many different indications, so procurement and availability have not been problematic, but resistance is more common than among aminoglycosides. Some foods or beverages and antacids high in content of divalent or 
trivalent cations can reduce fluoroquinolone absorption (16, 167-169). When feasible, taking fluoroquinolones on an empty stomach minimizes the potential of delays in or reduction of fluoroquinolone absorption. Moxifloxacin and to a lesser extent levofloxacin prolong the QT interval. Moxifloxacin may necessitate ECG monitoring, especially if patients have a baseline QTc > 500 milliseconds or take other QT-prolonging drugs.

Pharmacokinetic modeling studies for levofloxacin and moxifloxacin use in children are ongoing: recent data suggest that for children, a levofloxacin dose of at least 15 to $20 \mathrm{mg} / \mathrm{kg}$ daily $(170,171)$, and a moxifloxacin dose of 10 to $15 \mathrm{mg} / \mathrm{kg} / \mathrm{d}$ are effective (based on data showing too low exposure at $7.5-10 \mathrm{mg} / \mathrm{kg}$ ) (172). Although a modeling paper suggests a dose of 25 $\mathrm{mg} / \mathrm{kg} / \mathrm{d}$ for infants to 3 months of age and $20 \mathrm{mg} / \mathrm{kg} / \mathrm{d}$ for toddlers, safety at these doses has not been verified (173).

Conclusions. In our PS-matched IPDMA, patients treated with moxifloxacin and levofloxacin had better outcomes than patients not treated with any fluoroquinolones, or compared with patients treated with ofloxacin, after adjustment for numerous covariates that in themselves were strong determinants of outcome, such as clinical characteristics, extent of drug resistance, and number of other effective drugs in the treatment regimen. Moxifloxacin or levofloxacin should be included in a regimen to achieve a total of five effective drugs for the treatment of patients with MDR-TB. Our recommendation for the use of moxifloxacin or levofloxacin is strong despite very low certainty in the evidence, because we viewed the significant reduction in mortality, improved treatment success, and relatively few adverse effects associated with MDR-TB treatment that includes these latergeneration fluoroquinolones (compared with no fluoroquinolones) as having a particularly favorable balance of benefits over harms.

Research needs. Research is needed on the most effective doses (e.g., The OPTI-Q [Prospective, Randomized, Blinded Phase 2 Pharmacokinetic/Pharmacodynamic Study of the Efficacy and Tolerability of Increasing Doses of Levofloxacin in Combination with Optimized Background Regimen for the Treatment of MDR-TB (TBTC Study 32/ DMID Study 13-0057)] Trial; ClinicalTrials.gov Identifier:
PICO Question 12-Fluoroquinolones: In patients with MDR-TB, are outcomes safely improved when regimens include fluoroquinolones compared with regimens that do not include fluoroquinolones?

Recommendation 12: We recommend including moxifloxacin or levofloxacin in a regimen for treatment of patients with MDR-TB (strong recommendation, low certainty in the evidence). Our recommendation for the use of moxifloxacin or levofloxacin is strong despite very low certainty in the evidence because we viewed the significant reduction in mortality, improved treatment success, and relatively few adverse effects associated with MDR-TB treatment that includes these latergeneration fluoroquinolones (compared with no fluoroquinolones) as having a particularly favorable balance of benefits over harms.
NCT01918397) and dosing regimens, as preliminary work suggests these drugs may be more effective at higher doses (174). The activity, safety, and tolerability of using higher doses of fluoroquinolones against isolates with modestly elevated MICs should also be explored. Modest increases in toxicity can be minimized and managed by clinicians and may still be preferable to treating MDR-TB without quinolones. Higher doses may also limit acquired resistance $(175,176)$.

\section{Injectables: Amikacin, Capreomycin, Kanamycin, and Streptomycin}

The term "injectable drugs" in general encompasses four drugs: the aminoglycoside antibiotics streptomycin, amikacin, and kanamycin; and the cyclic polypeptide antibiotic capreomycin (155, 162, 177). Aminoglycosides are highly cationic and water soluble, but insoluble in organic solvents and hydrophobic environments, explaining much about their pharmacology, limited ability to cross lipid membranes, poor absorption from the gastrointestinal tract, and poor penetration into the CNS. Consequently, they are intravenous infusion or through intramuscular injection (177). Streptomycin's core ring structure differs administered parenterally through slow from all other aminoglycosides, explaining in part why cross-resistance between streptomycin and other aminoglycosides is uncommon $(155,177)$. These drugs block protein synthesis at the ribosomal level by binding to a highly conserved nucleotide sequence in the prokaryotic $16 \mathrm{~S}$ ribosomal subunit, the mRNA decoding region, where translation of mRNA codon to aminoacyltransfer RNA anticodon normally takes place $(155,177)$. Aminoglycosides share three important characteristics: 1 ) concentration-dependent killing, 2) a postantibiotic effect, and 3) synergism with other antibacterial drugs (155). These drugs kill bacteria in proportion to drug concentration, so a single daily dose is more effective than divided doses or a continuous infusion. Moreover, antibacterial activity continues many hours after serum levels become undetectable, the so-called "postantibiotic" effect (155).

Summary of the evidence. Procedures and methodology to assemble and rank the certainty in the evidence are reported in Appendix A, with evidence profiles for PICO questions reported in ApPENDIX B. In our PS-matched IPDMA, treatment outcomes for 613 patients who did not receive an injectable drug were compared with 1,554 patients treated with streptomycin, 4,330 treated with kanamycin, 2,275 treated
PICO Question 13-Injectables: In patients with MDR-TB, are outcomes safely improved when regimens include an injectable compared with regimens that do not include an injectable?

Recommendation 13: We suggest including amikacin or streptomycin in a regimen for treatment of patients with MDR-TB when susceptibility to these drugs is confirmed (conditional recommendation, very low certainty in the evidence). Because of their toxicity, these drugs should be used when more effective or less toxic therapies cannot otherwise be assembled to achieve a total of five effective drugs. We suggest NOT including kanamycin or capreomycin (conditional recommendation, very low certainty in the evidence). 
with amikacin, and 2,401 treated with capreomycin. Patients who received two or more injectable drugs $(n=875)$ were excluded, because the outcome could not be ascribed to one of the drugs (3). The noinjectable comparison group had notably fewer AFB smear-positive patients and patients with cavitary disease. On the other hand, patients in this group had more past treatment with second-line drugs; more resistance to quinolones, injectables, and XDR TB; and much more treatment with linezolid. In these respects, the capreomycin group was similar (more past treatment with second-line drugs, worse pretreatment drug resistance) to the other three drugs. Analysis was based on patients whose isolates were susceptible to the drug they received, with exceptions noted below.

Benefits. INJECTABLE DRUGS COMPARED WITH NO INJECTABLE DRUG. Compared with 613 patients treated without an injectable drug, 1,554 streptomycin-treated patients had increased treatment success (aOR, 1.5; 95\% CI, 1.1-2.1). In the subgroup with quinolone resistance (17.8\% of the total), streptomycin-treated patients had increased treatment success (aOR, 3.0; 95\% CI, 1.3-6.6). Compared with 613 patients not treated with injectable drugs, 2,275 amikacin-treated patients had increased treatment success (aOR, 2.0; 95\% CI, 1.5-2.6). In the subgroup with quinolone resistance, amikacin was also associated with increased treatment success (aOR, 3.0; 1.6-5.6). Among patients with XDR-TB, amikacin was associated with reduced deaths (aOR, 0.4; 95\% CI, 0.2-0.8). In contrast, neither kanamycin nor capreomycin was associated with any benefit on treatment success or death versus no injectable drug at all. To the contrary, kanamycin treatment was associated with fewer treatment successes (aOR, 0.5; 95\% CI, 0.4-0.6). Capreomycin was associated with an increased risk of death (aOR, 1.4; 95\% CI, 1.1-1.7). In XDR-TB, capreomycin was associated with increased deaths (aOR, 3.4; 95\% CI, 2.7-4.3) when compared with regimens with no injectable drug.

In a pediatric IPDMA, the use of second-line injectable agents (amikacin, kanamycin, capreomycin) in children with confirmed MDR-TB was associated with more treatment success compared with those not receiving second-line injectable agents (aOR, 2.94; 95\% CI, 1.05-8.28; $P=0.041$ ) (113). However, a high proportion of children with less-severe disease who received no second-line injectable agents still did well; therefore, children may be able to be spared from injectables and the associated toxicities if newer, more-effective drugs can be included in an all-oral regimen (113). INJECTABLE DRUGS COMPARED AGAINST EACH OTHER. Compared with streptomycintreated patients, patients treated with amikacin had increased treatment successes (aOR, 1.7; 95\% CI, 1.3-2.2) but with no significant impact on death (aOR, 1.0; 95\% CI, 0.8-1.2). Kanamycin and capreomycin were both significantly inferior to streptomycin in every respect. Amikacin was superior to kanamycin and capreomycin in every respect, with higher treatment success rates, lower death rates, or both. In the quinolone-resistant subgroup, on the other hand, use of amikacin did not have a significant effect on treatment success (aOR, 1.7; 95\% CI, 0.9-3.4) or death (aOR, 1.2; 95\% CI, 0.7-2.0) compared with use of streptomycin.

Harms. All aminoglycosides and capreomycin share important toxicities, especially nephrotoxicity, ototoxicity, and electrolyte disturbances, as well as other less common toxicities $(155,162,177)$. The ototoxicity can be vestibular, resulting in loss of balance, or cochlear, resulting in hearing loss. Because these drugs date back to the early years of antibiotic discovery and development, there is vast published experience with their toxicities. In the treatment of MDR-TB, the risk of toxicity is substantial, because the duration of treatment is many months. Ototoxicity may be severe and irreversible but with close monitoring can be minimized or prevented. Nephrotoxicity is often reversible when identified early and addressed appropriately; however, ototoxicity can progress even after the drug is stopped. Monitoring (measuring drug levels; monthly high-quality audiometry, electrolytes, and serum creatinine) and skilled management can prevent or mitigate these effects and are part of standard practice for MDR-TB experts. Risk of hearing loss increases with increasing duration of treatment. For aminoglycosides in general, the estimated frequency of nephrotoxicity is $5 \%$ to $15 \%$ and of ototoxicity $2 \%$ to $14 \%$, including $2 \%$ to $10 \%$ cochlear and $3 \%$ to $14 \%$ vestibular (155). In a survey of clinical trials performed between 1975 and 1982 and totaling $\sim 10,000$ patients, the incidence of amikacin nephrotoxicity was $8.7 \%$ (178). Cumulative dose and duration predicted toxicity; older age, dehydration/hypovolemia/hypotension, prior aminoglycoside treatment, coexisting hepatic or renal disease, and concomitant medications were important as well (155). Significant hearing impairment exceeds $50 \%$ in some reported series, renal dysfunction approaches $50 \%$, and vestibular dysfunction as high as $20 \%$ has been reported $(155,162,177)$. At the other extreme, series have been published with no significant or permanent toxicity, including with longer-term use $(155,162,177)$. Direct comparisons of drug toxicity for the four drugs used in TB are scarce. Experience suggests streptomycin may be more ototoxic, but that may be a consequence of far longer and wider use of streptomycin. Observational studies suggest amikacin may be more ototoxic than the other drugs (179-181).

In children, ototoxicity (hearing loss) has been documented in up to $24 \%$ of cases in a retrospective study, which also brings serious implications for development of normal speech (182). Pain of intramuscular injections can be safely reduced by adding lidocaine to amikacin injections without interference of pharmacokinetics (183). Dose range of amikacin should be 15 to $20 \mathrm{mg} / \mathrm{kg}$ as single daily dose.

Additional considerations. When injectables are used, serum creatinine, electrolyte measurements, clinical assessment for vertigo and tinnitus, highquality audiometry (including hearing frequency of $6,000-8,000 \mathrm{~Hz}$, as highfrequency hearing loss is seen initially), and clinical examinations should be conducted at least monthly, or more frequently if adverse effects occur. Limited data suggest there may be a genetic predisposition for hearing loss associated with specific mitochondrial gene mutations (184). Although routine genetic testing is not currently suggested, the provider should be aware of these genetic mutations and the risks of ototoxicity $(185,186)$. Because bactericidal activity is concentration dependent, high peak levels and single daily dosing are preferred to divided doses. Because of the postantibiotic effect, toxicity can be minimized by allowing trough levels between doses to remain below detectable levels for many hours. Some experts take advantage of these 
pharmacokinetic and pharmacodynamic properties with thrice-weekly dosing, especially after conversion of cultures to negative, decreasing healthcare system demands and the requirement for daily injections $(16,177)$. Intramuscular injections are painful, and 6 months of injections can be traumatic, especially to younger patients. Patients' values may differ sharply from providers in this respect and should be considered when determining whether to use injectable agents.

Conclusions. In our PS-matched IPDMA, the use of amikacin and streptomycin when the patient's isolate was susceptible to these drugs was associated with an increase in treatment success when compared with the control group not receiving these injectables. However, because of their toxicity and modest efficacy compared with other drugs, which also are less toxic, these drugs should be reserved for when more-effective or lesstoxic therapies cannot be assembled to achieve a total of five effective drugs. In our analyses, amikacin and streptomycin had similar aORs for treatment success in a minority of patients with fluoroquinolone resistance. Kanamycin and capreomycin were ineffective. We recommend against using kanamycin or capreomycin. As is the case for adults, the use of amikacin and streptomycin for children should also be reserved to when more-effective or less-toxic therapies cannot be assembled to achieve a total of five effective drugs.

Research needs. $N$-acetylcysteine, a thiol-containing antioxidant, may limit the severity and irreversibility of aminoglycoside-induced ototoxicity (187, 188), warranting additional research into this and other otoprotective measures that may improve the balance of benefits and harms for using these injectable agents.

\section{Linezolid}

Linezolid is an oxazolidinone antibiotic that inhibits bacterial protein synthesis by preventing the fusion of $30 \mathrm{~S}$ and $50 \mathrm{~S}$ ribosomal subunits (189). It also binds to human mitochondria and inhibits protein synthesis, which is the mechanism of toxicity in clinical use $(190,191)$.

Linezolid was initially used off-label in the absence of consistent scientific evidence as part of the regimen for difficult-to-treat cases of MDR- and XDR-TB (192). The first large retrospective observational study suggested linezolid was effective, but with frequent and often severe adverse events (192). The same study suggested, for the first time, that reducing the daily dose from $1,200 \mathrm{mg}$ to $600 \mathrm{mg}$ per day might be associated with fewer adverse events and improved tolerability. Several systematic reviews, IPDMAs, and one controlled clinical trial have been published on linezolid for treatment of MDR-TB (87, 193-196). The clinical trial confirmed previous observational findings and, in particular, the effectiveness of linezolid and its potential toxicity (195). A meta-analysis with 121 cases of patients with MDR-TB from 11 countries, treated with linezolid, confirmed linezolid effectiveness (culture conversion, 93.5\%; treatment success, $81.8 \%$ ) and that the 600 mg daily dose was safer than the 1,200-mg dose $(46.7 \%$ adverse events vs. $74.5 \%$, respectively) without lowering its effectiveness (196). The clinical trial confirmed the efficacy, safety, and tolerability of linezolid in patients with XDR-TB (194). There are insufficient data regarding the effectiveness of initiating treatment with doses $<600 \mathrm{mg}$ daily to recommend lower doses. Recently, the importance of therapeutic drug monitoring to reduce adverse events potentially due to linezolid has been emphasized (197, 198).

PICO Question 14-Linezolid: In patients with MDR-TB, are outcomes safely improved when regimens include linezolid compared with regimens that do not include linezolid?

Recommendation 14: We suggest including linezolid in a regimen for the treatment of patients with MDR-TB (conditional recommendation, very low certainty in the evidence). This is a conditional recommendation despite linezolid-containing regimens showing large reduction in mortality and improved treatment success, similar to bedaquiline and later-generation fluoroquinolones, because linezolid had more adverse effects and the balance of benefits and harms was less favorable compared with those drugs.

Summary of the evidence. Linezolid was used in regimens for MDR- and XDRTB across 38 studies (3). The initial dose of linezolid was $1,200 \mathrm{mg} / \mathrm{d}$ for 91 patients in five studies, $600 \mathrm{mg} / \mathrm{d}$ for 784 patients in 28 studies, and $300 \mathrm{mg} / \mathrm{d}$ for 99 patients in five studies (3). Procedures and methodology to assemble and rank the certainty in the evidence are reported in AppeNDIX A, with evidence profiles for PICO questions reported in APPENDIX $B$.

Benefits. In our PS-matched IPDMA, patients who received linezolid-containing regimens were more likely to achieve treatment success (aOR, 3.4; 95\% CI, 2.6-4.5) and to have a lower rate of death (aOR, 0.3; 95\% CI, 0.2-0.3) than those who did not receive linezolid. The effect on treatment success was more pronounced when studies from only high-income countries were included (aOR, 3.9; 95\% CI, 2.6-5.8). The greatest impact was found in patients with XDR-TB, where the aOR for successful treatment versus failure or relapse was 6.3 (95\% CI, 3.9-10.1) and the aOR for death was 0.1 (95\% CI, 0.1-0.2). When PS-matched pairs analyses were performed comparing the effects of bedaquiline and linezolid with those of no bedaquiline or linezolid, the combination of bedaquiline and linezolid was associated with an aOR of 2.7 (95\% CI, 1.5-4.9) for success versus failure/relapse, and of 0.3 (95\% CI, 0.2-0.4) for death versus success/failure/relapse. The efficacy of linezolid in children with TB has been shown in two studies of children $<18$ years of age, albeit with few patients $(199,200)$.

Harms. Adverse effects associated with linezolid in patients with TB include neurotoxicity (i.e., peripheral neuropathy and optic neuritis), myelosuppression, hyperlactatemia, and diarrhea, all of which are presumably secondary to the inhibition of mitochondrial protein synthesis (190, 201). A published systematic review of 12 studies conducted in 11 countries globally reported an adverse event rate of 58.9\% (hematological, neurological, and gastrointestinal), predominantly noted in individuals treated with a dosages $>600$ $\mathrm{mg} / \mathrm{d}$ (196). Hematological toxicity can occur quickly after starting treatment and can involve any cell line. Neurotoxicity, including optic neuritis and peripheral neuropathy, occur later, usually after 12 to 20 weeks of treatment. Toxicity has been associated with trough levels of $>2.0$ 
PICO Question 15-Macrolides: In patients with MDR-TB, are outcomes safely improved when regimens include macrolides compared with regimens that do not include macrolides?

Recommendation 15: We recommend NOT including the macrolides azithromycin and clarithromycin in a treatment regimen for patients with MDR-TB (strong recommendation, very low certainty in the evidence). Our recommendation against the use of the macrolides azithromycin and clarithromycin in MDR-TB treatment is strong despite the evidence being judged to be of very low certainty because we viewed the increased mortality and decreased likelihood of treatment success associated with the use of this drug class as having a notably unfavorable balance of benefits to potential harms. (see Role of Therapeutic Drug Monitoring in Treatment of MDR-TB) (202-204). Adverse effects, especially myelosuppression, are also noted to be common at the currently recommended dose of $10 \mathrm{mg} / \mathrm{kg}$ twice daily in children $<10$ years of age.

Additional considerations. Although use of linezolid for the FDA-approved 28 days or less for non-TB indications is associated with an acceptable adverse effect profile (205), the data on the longer-term use necessary for MDR-TB are limited (3, $195,200)$. Strict clinical monitoring for potential toxicity (in particular peripheral neuropathy, optic neuritis, anemia, and leukopenia) is necessary because of the risk of adverse events associated with the longterm use of linezolid (16). If optic neuritis occurs, many patients may be rechallenged successfully with linezolid once vision normalizes. Assessment for visual toxicity must continue after restarting linezolid. Some patients are able to be rechallenged with the full dose; others are able to avoid recurrent visual toxicity with a reduced dose of linezolid at $300 \mathrm{mg}$ daily (195). Linezolid should generally not be administered to patients taking serotonergic agents, such as monoamine oxidase inhibitors, because of the potential for serious CNS reactions, such as serotonin syndrome. Because monoamine oxidase type A deaminates serotonin, and selective serotonin reuptake inhibitors potentiate the action of serotonin by inhibiting its neuronal reuptake, administration of linezolid concurrently with a selective serotonin reuptake inhibitor can lead to serious reactions, such as serotonin syndrome or neuroleptic malignant syndrome-like reactions (16). One randomized clinical trial demonstrated that lowering the dose from $600 \mathrm{mg} / \mathrm{d}$ to 300 $\mathrm{mg} / \mathrm{d}$ after culture conversion reduced toxicity (195). For children, one modeling study reported that linezolid dose of 15 $\mathrm{mg} / \mathrm{kg}$ in full-term neonates and infants aged $\leqslant 3$ months and $10 \mathrm{mg} / \mathrm{kg}$ in toddlers, administered once daily, achieved cumulative fraction of response of $\geqslant 90 \%$, with $<10 \%$ achieving linezolid area under the concentration-versus-time curve (AUC) of 0 to 24 associated with toxicity (173). On the basis of modeling of pharmacokinetic data from 48 children, WHO and Sentinel Project recommend pediatric doses of linezolid as $15 \mathrm{mg} / \mathrm{kg}$ once daily for children $<15 \mathrm{~kg}$ and 10 to $12 \mathrm{mg} / \mathrm{kg}$ once daily for those weighing $>15 \mathrm{~kg}(7,17)$. It is common practice for patients on linezolid to be prescribed vitamin B6 (16).

Conclusions. In our PS-matched IPDMA, patients who received linezolidcontaining regimens were more likely to achieve treatment success and to have a lower rate of death than those who did not receive linezolid. We suggest including linezolid in a regimen for the treatment of
PICO Question 16-p-Aminosalicylic Acid: In patients with MDR-TB, are outcomes safely improved when regimens include $p$-aminosalicylic acid compared with regimens that do not include $p$-aminosalicylic acid?

Recommendation 16: We suggest NOT including $p$-aminosalicylic acid in a treatment regimen for patients with MDR-TB (conditional recommendation, very low certainty in the evidence). When the individualized treatment regimen for patients with MDR-TB contains newer-generation, more-effective drugs, the addition of $p$-aminosalicylic acid does not appear to provide a benefit. patients with MDR-TB. This is a conditional recommendation despite linezolid-containing regimens showing large reduction in mortality and improved treatment success, similar to bedaquiline and later-generation fluoroquinolones, because linezolid had more adverse effects and the balance of benefits and harms was less favorable compared with those drugs.

Research needs. Clinical trials of combinations of new chemical entities plus linezolid, including when administered at different doses and durations to optimize its therapeutic effect while minimizing toxicity are underway (Nix-TB, ClinicalTrials.gov Identifier: NCT02333799; ZeNix [Safety and Efficacy of Various Doses and Treatment Durations of Linezolid Plus Bedaquiline and Pretomanid in Participants with Pulmonary TB, XDR-TB, Pre-XDRTB or Non-responsive/Intolerant MDRTB], NCT03086486). Further linezolid pharmacokinetic and safety data are needed to find optimal dosing in adults and children.

\section{Macrolides: Azithromycin and Clarithromycin}

The macrolides azithromycin and clarithromycin have unclear efficacy and role in the treatment of MDR-TB (23). Macrolides are commonly used to treat upper and lower respiratory tract infections and have an essential role in the treatment of nontuberculous mycobacteria (206). They are believed to have immunomodulatory and antiinflammatory effects. M. tuberculosis has intrinsic, inducible resistance to clarithromycin (207, 208), and in vivo murine TB models confirm the lack of activity of macrolides (209). Clarithromycin may increase linezolid serum exposure when used in combination (210), prompting some consideration of potential synergy between macrolides and other MDR-TB drugs (211). WHO does not recommend use of macrolides to treat MDR-TB (23).

Summary of the evidence. Procedures and methodology to assemble and rank the certainty in the evidence are reported in APPENDIX A, with evidence profiles for PICO questions reported in APPENDIX B. In our PSmatched IPDMA, patients who received macrolides $(n=1,067)$ were more likely to have been treated with second-line drugs and to have resistance to fluoroquinolones or any 
second-line injectable (3). Patients who received macrolides were more likely to receive later-generation fluoroquinolones, capreomycin, and linezolid. In adjusted analyses, patients who received macrolides were less likely to achieve treatment success (aOR, 0.6; 95\% CI, 0.5-0.8) and had a higher rate of death $(\mathrm{aOR}, 1.6,95 \% \mathrm{CI}$, 1.2-2.0) than patients who did not receive macrolides.

Benefits. The available evidence does not support the use of macrolides in the treatment of MDR-TB.

Harms. In our PS-matched IPDMA, patients who received macrolides were less likely to achieve treatment success (aOR, 0.6; 95\% CI, 0.5-0.8) and had a higher rate of death $(\mathrm{aOR}, 1.6 ; 95 \% \mathrm{CI}$, $1.2-2.0$ ) than patients who did not receive macrolides.

Conclusions. Macrolides, specifically azithromycin or clarithromycin, should not be included in a regimen for the treatment of patients with MDR-TB. Our recommendation against the use of the macrolides azithromycin and clarithromycin in MDR-TB treatment is strong despite the evidence being judged to be of very low certainty because we viewed the increased mortality and decreased likelihood of treatment success associated with the use of this drug class as having a notably unfavorable balance of benefits to potential harms.

Research needs. Further research may be warranted on newer-generation macrolides and to elucidate if there is potential synergy of macrolides with linezolid or other second-line agents.

\section{p-Aminosalicylic Acid}

One of the first agents found to be effective against TB (212), $p$-aminosalicylic acid has been widely used clinically, although its precise mode of action remains uncertain (213). With the discovery of other more potent drugs, including rifampin, $p$-aminosalicylic acid, initially used combined with streptomycin, was no longer considered in first-line regimens. It is now used as part of a treatment regimen for MDR- and XDR-TB, although the benefits of $p$-aminosalicylic acid are not clear and toxicity limits its use. Current guidance recommends that $p$-aminosalicylic acid be used to compose a regimen when five effective drugs cannot otherwise be assembled $(16,23)$.
Summary of the evidence. Procedures and methodology to assemble and rank the certainty in the evidence are reported in Appendix A, with evidence profiles for PICO questions reported in ApPENDIX B. In our PS-matched IPDMA, $p$-aminosalicylic acid was not associated with any benefit on treatment success $(\mathrm{aOR}, 0.8$; 95\% CI, $0.7-1.0)$ and was associated with increased death (aOR, 1.2; 95\% CI, 1.1-1.4) (3). The potential to cause adverse events $(12.2 \%$ in a previous meta-analysis) has also limited the tolerability of $p$-aminosalicylic acid (23).

Benefits. No association with any benefit on treatment success was identified for $p$-aminosalicylic acid in our PS-matched IPDMA. In an IPDMA of children with MDR-TB, there was no benefit identified for the inclusion of $p$-aminosalicylic acid in regimens (aOR, 0.75; 95\% CI, 0.25-1.96; $P=0.483)$ (113).

Harms. Gastrointestinal distress is common with $p$-aminosalicylic acid but reported to occur less with the PASER formulation than with older preparations (16). Rare hepatotoxicity and thrombocytopenia have been reported, as has reversible hypothyroidism, particularly when used concomitantly with ethionamide (120). In the setting of hypothyroidism, some experts provide thyroid replacement therapy rather than discontinuing $p$-aminosalicylic acid. Clinical experience has shown $p$ aminosalicylic acid to be better tolerated regarding gastrointestinal disturbance in children than in adults, although hypothyroidism also remains common in children.

Additional considerations. Indirect comparison suggests that ethionamide may be better than $p$-aminosalicylic acid if a fifth drug is needed to construct a regimen with at least five effective drugs. Old studies have shown $p$-aminosalicylic acid to be a good companion drug to protect other drugs from developing resistance and also have shown efficacy in combination with first-line drugs (214, 215). It has recently been suggested by pediatric DR-TB experts that in children $p$-aminosalicylic acid may replace an injectable agent in the absence of better drugs (83). When $p$-aminosalicylic acid is used, experts recommend monitoring thyroid-stimulating hormone, electrolytes, blood counts, and liver function tests.
PICO Question 17-Pyrazinamide: In patients with MDR-TB, are outcomes safely improved when regimens include pyrazinamide compared with regimens that do not include pyrazinamide?

Recommendation 17: We suggest including pyrazinamide in a treatment regimen for patients with MDR-TB, when the M. tuberculosis isolate has not been found to be resistant to pyrazinamide (conditional recommendation, very low certainty of evidence).

Conclusions. When the individualized treatment regimen for patients with MDR-TB contains newer-generation, more-effective drugs, the addition of $p$-aminosalicylic acid does not appear to provide benefit.

Research needs. Given the limited armament of TB drugs available for treating MDR-TB, some experts have advocated for the evaluation of doseoptimized $p$-aminosalicylic acid to improve efficacy while minimizing toxicity (216). Research on whether p-aminosalicylic acid, among other agents, may offer protection against the acquisition of resistance to other drugs in the regimen would also be valuable.

\section{Pyrazinamide}

Pyrazinamide, a nicotinamide analog, is a prodrug that is converted in vivo into pyrazinoic acid, which interferes with mycobacterial fatty acid synthase. Pyrazinamide has demonstrated effectiveness against $M$. tuberculosis; it is included in standard regimens for treatment of drug-susceptible TB and also used in regimens for MDR-TB $(11,16,23)$. However, recent population-based studies conducted as part of multicountry surveillance activities have shown that pyrazinamide resistance is highly associated with rifampin resistance $(217,218)$. This finding, in conjunction with evidence that pyrazinamide efficacy is reduced in the setting of pncA gene mutations (219-222), underscores the importance of documenting drug susceptibility to pyrazinamide by WGS, molecular tests, or traditional DST, if the drug is to be included as part of a regimen for MDR-TB. 
Table 9. Doses of Drugs for Treatment of Adults and Children with Multidrug-Resistant Tuberculosis

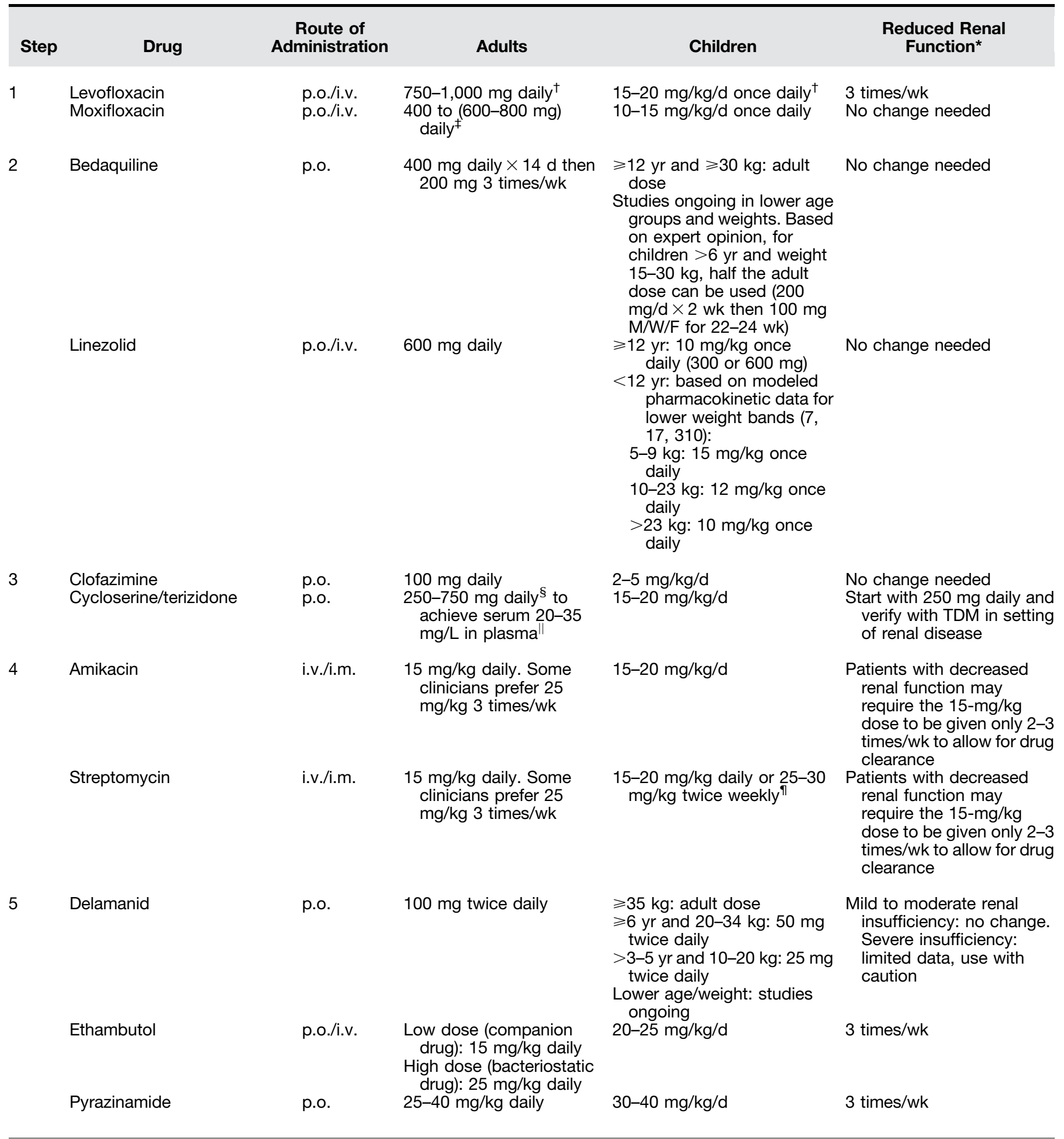


Table 9. (Continued)

\begin{tabular}{|c|c|c|c|c|c|}
\hline Step & Drug & $\begin{array}{c}\text { Route of } \\
\text { Administration }\end{array}$ & Adults & Children & $\begin{array}{l}\text { Reduced Renal } \\
\text { Function* }\end{array}$ \\
\hline \multirow[t]{7}{*}{6} & Ethionamide & p.o. & $\begin{array}{l}15-20 \mathrm{mg} / \mathrm{kg} \text { total (usually } \\
250-500 \mathrm{mg} \text { once or } \\
\text { twice daily) }\end{array}$ & $\begin{array}{l}15-20 \mathrm{mg} / \mathrm{kg} \text { total (divided } \\
1-2 \text { times/d) }\end{array}$ & No change needed \\
\hline & Prothionamide & p.o. & $\begin{array}{l}15-20 \mathrm{mg} / \mathrm{kg} \text { total (usually } \\
250-500 \mathrm{mg} \text { once or } \\
\text { twice daily) }\end{array}$ & $\begin{array}{l}15-20 \mathrm{mg} / \mathrm{kg} \text { total (divided } \\
1-2 \text { times/d) }\end{array}$ & No change needed \\
\hline & Imipenem-cilastatin & i.v. & 1,000 mg 3-4 times/d & $\begin{array}{l}\text { (imipenem component) } \\
15-25 \mathrm{mg} / \mathrm{kg} / \mathrm{dose} 4 \\
\text { times } / \mathrm{d}\end{array}$ & May reduce frequency \\
\hline & Meropenem & i.v. & $1,000 \mathrm{mg} 3$ times/d $\mathrm{d}^{\dagger \dagger}$ & $20-40 \mathrm{mg} / \mathrm{kg} / \mathrm{dose} 3$ times $/ \mathrm{d}$ & May reduce frequency \\
\hline & $\begin{array}{l}\text { Clavulanate (component } \\
\text { of amoxicillin- } \\
\text { clavulanate) for } \\
\text { coadministration with } \\
\text { carbapenems } \\
\text { (imipenem-cilastatin } \\
\text { and meropenem) }\end{array}$ & p.o./i.v. & $250 \mathrm{mg} 3$ times/d & $\begin{array}{l}25 \mathrm{mg} / \mathrm{kg} / \mathrm{dose} \text { of amoxicillin } \\
\text { component } 3 \text { times } / \mathrm{d}\end{array}$ & $\begin{array}{l}\text { May reduce frequency to } \\
\text { match carbapenem }\end{array}$ \\
\hline & $p$-Aminosalicylic acid & p.o./i.v. & $4 \mathrm{~g} \mathrm{2-3} \mathrm{times/d^{ \ddagger \ddagger }}$ & $\begin{array}{l}200-300 \mathrm{mg} / \mathrm{kg} / \mathrm{d} \text { in two } \\
\text { divided doses } \\
\S \S\end{array}$ & No change needed \\
\hline & High-dose isoniazid ${ }^{||||}$ & p.o./i.v. & $15 \mathrm{mg} / \mathrm{kg}$ daily & $15-20 \mathrm{mg} / \mathrm{kg} / \mathrm{d}$ & No change needed \\
\hline
\end{tabular}

Definition of abbreviations: M/W/F = Monday/Wednesday/Friday; TDM=therapeutic drug monitoring.

Updated and modified from References 11, 16, 343, and 344.

*Dosages may not apply to patients with severely decreased kidney function, including in the setting of dialysis, for which consultation with a nephrologist is advised.

'Levofloxacin doses of up to $1,250 \mathrm{mg}$ have been used safely when needed to achieve therapeutic concentrations. A recent population pharmacokinetic study in South African children found that higher levofloxacin doses from $18 \mathrm{mg} / \mathrm{kg} / \mathrm{d}$ for younger children, up to $40 \mathrm{mg} / \mathrm{kg} / \mathrm{d}$ for older children, may be required to achieve adult-equivalent exposures (170).

FHigher moxifloxacin doses have been used safely when the isolate is resistant to ofloxacin and the minimum inhibitory concentration for levofloxacin or moxifloxacin suggests higher doses may overcome resistance. Higher doses also are used in cases of malabsorption.

${ }^{\S}$ Cycloserine doses can be divided if needed (typically twice daily). Doses $>750 \mathrm{mg}$ are difficult for many patients to tolerate.

"Cycloserine dose may be lowered if serum concentrations exceed $35 \mu \mathrm{g} / \mathrm{ml}$, even if patient is not experiencing toxicity, to prevent central nervous system toxicity.

"Modified from adult intermittent dose of $25 \mathrm{mg} / \mathrm{kg}$, and accounting for larger total body water content and faster clearance of injectable drugs in most children. Dosing can be guided by serum concentrations.

${ }^{* *}$ Ethionamide can be given at bedtime or with a meal to reduce nausea. Experienced clinicians suggest starting with $250 \mathrm{mg}$ once daily and gradually increasing the dose over 1 week. Serum concentrations may be useful in determining the appropriate dose. Few patients tolerate 500 mg twice daily.

${ }^{+}$Studies are ongoing evaluating meropenem at higher doses (ClinicalTrials.gov identifiers: NCT03174184 and NCT02349841).

拉Some experts prescribe $p$-aminosalicylic acid at $6 \mathrm{~g}$, and up to12 $\mathrm{g}$, administered once daily $(16,216)$.

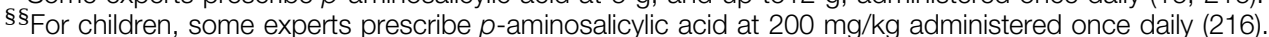

IIIIIsoniazid is tested at two concentrations. Some experts use these results (or resistance conferred through mutations in inhA) to select a higher dose when it tests resistant at the lower concentration and susceptible at the higher concentration. The higher dose may achieve in vivo concentrations sufficiently high to overcome low-level resistance $(16,216)$.

Pyrazinamide has been demonstrated to have substantial sterilizing activity as part of combination regimens and has allowed for treatment shortening in drugsusceptible TB $(223,224)$. Higher doses have been shown to be more effective in animal models and phase $2 \mathrm{~A}$ studies, but doses of 40 to $70 \mathrm{mg} / \mathrm{kg}$ were found to be too toxic to be pursued further in human studies (224).

Summary of the evidence. Procedures and methodology to assemble and rank the certainty in the evidence are reported in Appendix A, with evidence profiles for PICO questions reported in Appendix B. Our PS-matched IPDMA compared outcomes in 1,986 individuals with isolates susceptible to pyrazinamide who received the drug with 307 individuals with isolates susceptible to pyrazinamide who did not receive the drug (3). However, the groups were not balanced with regard to other effective drugs; in particular, the patients not receiving pyrazinamide were more likely to receive linezolid and a latergeneration fluoroquinolone. Thus, the ability to detect an effect of pyrazinamide might have been partially obscured. In a previous IPDMA, before linezolid and bedaquiline were more commonly used, administration of pyrazinamide among patients with susceptible isolates was associated with an aOR of 1.9 (95\% CI, 1.3-2.9) for cure/complete versus failure/relapse and an aOR of 1.6 (95\% CI, 1.3-2.1) for cure/complete versus failure/relapse/death, compared with patients receiving pyrazinamide whose isolates were resistant in vitro (6).

Benefits. In our PS-matched IPDMA, treatment success was significantly less likely with regimens containing pyrazinamide (aOR, 0.7; 95\% CI, 0.5-0.9), but death was also significantly less frequent ( $\mathrm{aOR}, 0.7$; 95\% CI, 0.6-0.8). This paradox may be due to confounding in our PS-matched IPDMA, as patients who did not receive pyrazinamide were substantially more likely to receive linezolid. Moreover, our PS-matched IPDMA did not assess the potential ability of pyrazinamide to 
contribute to treatment shortening, as has been achieved in drug-susceptible TB. Because pyrazinamide is associated with increased success only when used to treat patients whose isolates are susceptible to the drug (144), whenever feasible, the decision to include pyrazinamide in a regimen should be based on pyrazinamide susceptibility results.

In an IPDMA of children with MDR$\mathrm{TB}$, the addition of pyrazinamide to a regimen showed no benefit in the treatment of confirmed MDR-TB cases (aOR, 1.63; 95\% CI, 0.41-6.56; $P=0.484$ ) (113).

Pyrazinamide resistance was, however, not tested and improved selection of cases on the basis of resistance might change this outcome.

Harms. Pyrazinamide has been used extensively in the treatment of $\mathrm{TB}$, and toxicities are well documented (225). The most common is gastrointestinal upset or intolerance. In a recent review of the tolerability of TB drugs, pyrazinamide was associated with serious adverse events in 56 of 2,023 (2.8\%) patients (226). This is consistent with previous reports of pyrazinamide toxicity $(11,225,227)$. Hepatic enzyme elevations are common with pyrazinamide, and significant hepatotoxicity, although less common, can occur. Modest elevated serum uric acid levels are also expected, although the clinical significance of this is unclear. Nongouty polyarthralgias and

hypersensitivity reactions can occur. Flares of clinical gout can also occur, especially in those with a history of prior gouty arthritis.

Additional considerations. All patients receiving pyrazinamide as part of an MDRTB treatment regimen should be monitored carefully for signs or symptoms of hepatotoxicity and have their pyrazinamide dose held or decreased if such toxicity is detected. Isolated increases in uric acid without symptoms of gout are common and are not an indication to discontinue the drug. Recent population-based studies have found that pyrazinamide resistance is common in the setting of MDR with some regional variability, suggesting that pyrazinamide susceptibility should be confirmed or suspected if the drug is included in the regimen $(217,228,229)$. Although there are known challenges related to accurately determining phenotypic DST for pyrazinamide, recent highly predictive DNA sequencing techniques show significant promise for newer genomic approaches (230).
When included in the regimen, most experts use doses of 25 to $40 \mathrm{mg} / \mathrm{kg} / \mathrm{d}$ orally. The recommended dose of pyrazinamide in children is 30 to 40 $\mathrm{mg} / \mathrm{kg}$ daily.

Conclusions. We suggest including pyrazinamide in a regimen for the treatment of patients with MDR-TB, when the $M$. tuberculosis isolate has not been found resistant to pyrazinamide.

Research needs. Pyrazinamide is being evaluated as part of novel treatment regimens for both DS and MDR-TB in multiple clinical trials (56). Development of a reliable, simple molecular test for pyrazinamide susceptibility is a critically important research need. Dose optimization studies for pyrazinamide are warranted, as higher doses may be more efficacious but also may be more toxic.

\section{Building a Treatment Regimen for MDR-TB}

The guideline committee proposes a clinical strategy tool for building a treatment regimen for MDR-TB (Table 10). The clinical strategy tool incorporates the evidence-based review of the individual drugs, with consideration of the balance of benefits and harms for each drug, the experience of MDR-TB experts on the committee, as well as perspectives of patients. This clinical strategy tool encourages the building of all oral regimens with five effective drugs (to which the isolate is susceptible or has low likelihood of resistance) for the treatment of MDRTB. In our PS-matched IPDMA, significant favorable synergies were identified with improved treatment success and reduced mortality when bedaquiline was used in combination with linezolid or clofazimine. As noted, amikacin and streptomycin show modest effectiveness when the patient's isolate is susceptible to these drugs; however, because of their significant toxicities, aminoglycosides should be reserved for when a more-effective or lesstoxic regimen cannot otherwise be assembled. The final choice of drugs and drug classes is contingent on many factors, including patient preferences, harms and benefits associated with agents, the capacity to appropriately monitor for significant adverse effects, consideration of drug-drug interactions, comorbidities, and drug availability. Final regimen development, therefore, is individualized and may differ substantially from the approach described in Table 10. Doses of the drugs for treating adults and children with MDR-TB are provided in Table 9, modified and updated from the 2016 ATS/CDC/IDSA Treatment of Drug-Susceptible TB Practice Guidelines (11).

\section{Role of Therapeutic Drug Monitoring in Treatment of MDR-TB}

Specific pharmacokinetic (PK)/

pharmacodynamic (PD) targets, especially the AUC divided by the MIC over 24 hours $\left(\mathrm{AUC}_{0-24} / \mathrm{MIC}\right)$, are being recognized as playing an important role in determining efficacy (231). This has been demonstrated most clearly in hollow fiber systems in vitro that isolate the activity of the drug against the organism $(232,233)$. Further evidence has been provided by animal models (mouse, rabbit, nonhuman primates) and through human clinical trials (234-237). Drug levels are usually chosen to be four to five times greater than the MIC. Because of the complexities of clinical disease, it is more challenging to isolate the $\mathrm{PK} / \mathrm{PD}$ contribution of individual drugs, but studies have nonetheless been informative on the relationships between efficacy and drug exposure (238-240). In human $\mathrm{TB}$, a combination of drugs is used, and each patient has his or her unique duration of disease, host genetics, and particular strain of $M$. tuberculosis. The general term for these $\mathrm{PK} / \mathrm{PD}$ data is "exposure-response" data (i.e., for a given amount of drug exposure, how much response can you expect?). Much of the published data focus on the first-line TB drugs, with some emerging data becoming available for second-line drugs (241-243).

In clinical practice, the actual MIC for each drug often is not available.

Epidemiological cut-off values or "critical concentrations" that separate wild-type from more resistant isolates can be used for selecting what drugs to include in a regimen (244). These in vitro cut-offs are based on patterns of susceptibility compared with achievable concentrations within humans. An organism is not just "susceptible" as an inherent property; it is susceptible to inhibition or killing by specific, tested concentrations of the 
Table 10. Clinical Strategy to Build an Individualized Treatment Regimen for MDR-TB

- Build a regimen using five or more drugs to which the isolate is susceptible (or has low likelihood of resistance), preferably with drugs that have not been used to treat the patient previously.

- Choice of drugs is contingent on capacity to appropriately monitor for significant adverse effects, patient comorbidities, and preferences/values (choices therefore subject to program and patient safety limitations).

- In children with TB disease who are contacts of infectious MDR-TB source cases, the source case's isolate DST result should be used if an isolate is not obtained from the child.

- TB expert medical consultation is recommended (ungraded good practice statement).

Step 1: Choose one later-generation fluoroquinolone

Step 2: Choose both of these prioritized drugs

Step 3: Choose both of these prioritized drugs

Step 4: If a regimen cannot be assembled with five effective oral drugs, and the isolate is susceptible, use one of these injectable agents*

Step 5: If needed or if oral agents preferred over injectable agents in Step 4, use the following drugs ${ }^{\dagger}$

Step 6: If limited options and cannot assemble a regimen of five effective drugs, consider use of the following drugs

\section{The following drugs are no longer recommended for inclusion in MDR-TB regimens:}

\author{
Levofloxacin \\ Moxifloxacin \\ Bedaquiline \\ Linezolid \\ Clofazimine \\ Cycloserine/terizidone
}

Amikacin

Streptomycin

\section{Delamanid ${ }^{\ddagger}$ \\ Pyrazinamide \\ Ethambutol}

\author{
Ethionamide or prothionamide ${ }^{\S}$ \\ Imipenem-cilastatin/clavulanate or meropenem/clavulanate \\ p-Aminosalicylic acid ${ }^{\text {I }}$ \\ High-dose isoniazid ${ }^{\star *}$ \\ Capreomycin and kanamycin \\ Amoxicillin/clavulanate (when used without a carbapenem) \\ Azithromycin and clarithromycin
}

Definition of abbreviations: DST = drug susceptibility testing; INH = isoniazid; IPDMA = individual patient data meta-analyses; MDR= multidrug-resistant; $\mathrm{PS}=$ propensity score; $\mathrm{TB}=$ tuberculosis

*Amikacin and streptomycin should be used only when the patient's isolate is susceptible to these drugs. Because of their toxicity, these drugs should be reserved for when more-effective or less-toxic therapies cannot be assembled to achieve a total of five effective drugs.

${ }^{\dagger}$ Patient preferences in terms of the harms and benefits associated with injectables (the use of which is no longer obligatory), the capacity to appropriately monitor for significant adverse effects, consideration of drug-drug interactions, and patient comorbidities should be considered in selecting Step 5 agents over injectables. Ethambutol and pyrazinamide had mixed/marginal performance on outcomes assessed in our PS-matched IPDMA; however, some experts may prefer these drugs over injectable agents to build a regimen of at least five effective oral drugs. Use pyrazinamide and ethambutol only when the isolate is documented as susceptible.

${ }^{\ddagger}$ Data on dosing and safety of delamanid are available in children $\geqslant 3$ years of age.

$\$$ Mutations in the inhA region of the Mycobacterium tuberculosis genome can confer resistance to ethionamide/prothionamide as well as to INH. In this situation, ethionamide/prothionamide may not be a good choice unless the isolate is shown to be susceptible with in vitro testing.

"Divided daily intravenous dosing limits feasibility. Optimal duration of use not defined.

"Fair/poor tolerability and low performance. Adverse effects reported to be less common in children.

**Data not reviewed in our PS-matched IPDMA, but high-dose isoniazid can be considered despite low-level isoniazid resistance but not with high-level INH resistance.

drugs. Individual MIC values might be preferred; however, in practice, there are technical and financial barriers to such individualized data. Following standardized dosing, clinical experience over the past 3 decades clearly shows that some patients have low drug concentrations, leading to clinical failures $(11,231,241,242,244)$.

Many MDR-TB experts use therapeutic drug monitoring (TDM) to identify patients with problems with drug absorption, thereby informing dose adjustments.
Individuals who have poor response to $\mathrm{TB}$ treatment despite adherence may benefit from TDM (11). Patients with TB with gastrointestinal problems that increase risk of malabsorption, concurrent HIV infection, impaired renal clearance, or diabetes should be prioritized for TDM (11). Furthermore, some experts use TDM for all patients being treated for MDR or XDR-TB early in treatment, rather than waiting for a poor response. TDM should be used and interpreted in consultation with an expert in MDR-TB.
TDM also provides patient-specific information that may help limit toxicity due to certain drugs, including the injectable drugs cycloserine and linezolid (245-247). In particular, linezolid toxicity is associated with elevated trough values (202-204). "Target" ranges for the injectable drugs and cycloserine have been proposed, and research continues on refining these. Fluoroquinolones display concentrationdependent efficacy, and higher doses of moxifloxacin and levofloxacin are being studied $(174,248)$. Currently, 
avoiding low serum concentrations seems advisable.

A common clinical practice is to collect samples at 2 and 6 hours after drug administration to measure concentrations that may distinguish normal drug absorption (a 2 -h value within the normal range) from delayed absorption (a 6-h value greater than the 2 -h value, approaching the normal range) and malabsorption (both values are below the normal range). This approach also works for injectable drugs. Furthermore, trough values for linezolid can be helpful $(11,231,241,242,244)$. Although it is clearly possible to cure patients without TDM and even in the setting of lower serum drug concentrations, the available data suggest that the probability of cure decreases with decreasing drug concentrations (231-241). Given the incomplete information currently available, many expert clinicians use in vitro susceptibility data and TDM as available tools for optimizing the treatment of patients with MDR- and XDR-TB. For all patients, but in particular those receiving outpatient treatment, TDM should be coordinated and conducted using patientcentered approaches.

\section{Shorter-Course, Standardized, 9- to 12-Month Regimen for MDR-TB}

A randomized, phase 3, noninferiority trial, STREAM Stage 1 (ClinicalTrials.gov identifier: NCT02409290) was recently conducted to assess a shorter-course regimen composed of existing drugs for MDR-TB (8). This regimen had achieved success rates of $83 \%$ (95\% CI, 71.0-90.3\%) in cohort studies $(249,250)$. The shortercourse regimen is standardized and composed of kanamycin, moxifloxacin (in place of gatifloxacin, which was the fluoroquinolone originally used in the "Bangladesh" regimen), prothionamide, clofazimine, pyrazinamide, high-dose isoniazid, and ethambutol for an initial period (4-6 mo) and moxifloxacin, clofazimine, pyrazinamide, and ethambutol for the continuation phase (5 mo) (23). The total duration of therapy is between 9 and 11 months, compared with an individualized regimen of 18 to 24 months of therapy. The medication costs of the shorter regimen are believed to be less than that of conventional regimens, currently

PICO Question 18-Shorter-course, standardized regimen: In patients with MDR-TB, does treatment with a standardized MDR-TB regimen for $\leqslant 12$ months lead to better outcomes than treatment with an MDR-TB regimen for 18-24 months?

Recommendation 18: The shorter-course regimen is standardized with the use of kanamycin (which the committee recommends against using) and includes drugs for which there is documented or high likelihood of resistance (e.g., isoniazid, ethionamide, pyrazinamide). Although the STREAM Stage 1 randomized trial found the shorter-course regimen to be noninferior to longer injectable-containing regimens with respect to the primary efficacy outcome (8), the guideline committee cannot make a recommendation either for or against this standardized shorter-course regimen, compared with longer individualized all-oral regimens that can be composed in accordance with the recommendations in this practice guideline. We make a research recommendation for the conduct of randomized clinical trials evaluating the efficacy, safety, and tolerability of modified shorter-course regimens that include newer oral agents, exclude injectables, and include drugs for which susceptibility is documented or highly likely.

undergoing a formal economic evaluation as part of the STREAM trials (251).

\section{Summary of the Evidence}

In the STREAM Stage 1 clinical trial, of 424 participants who underwent randomization, 383 were included in the modified intention-to-treat population (8). Favorable status was reported in $79.8 \%$ of participants in the long-regimen group and in $78.8 \%$ of those in the short-regimen group-a difference, with adjustment for HIV status, of 1.0 percentage point $(95 \% \mathrm{CI}$,

-7.5 to 9.5$)$. The results with respect to noninferiority were consistent among the 321 participants in the per-protocol population. Procedures and methodology to assemble and rank the certainty in the evidence are reported in ApPENDIX A, with evidence profiles for PICO questions reported in ApPENDIX B. In our PS-matched IPDMA of 12,030 patients ( $n=50$ studies), 169 ( $n=33$ studies) were eligible for analysis of death and 1,369 ( $n=33$ studies) were eligible for analysis of treatment success (249) once the criteria used for the shorter-course regimen were applied (249). There were data from 532 individuals $(n=3$ studies) on the shorter regimen available for analysis of death and 498 ( $n=3$ studies) for analysis of treatment success. After using PS matching to adjust for age, sex, HIV, smear status, past TB treatment with first-line drugs, and number of effective drugs, there were no statistically significant associations for the shorter regimen with treatment success (aOR, 0.5; 95\% CI, 0.02-13), or deaths (aOR, 1.7; 95\% CI,
0.6-4.6), compared with individualized regimens.

\section{Benefits}

The shorter regimen allows for a significantly shorter duration of therapy compared with the individualized regimen and consequently less pill burden, medication cost, and associated provider administration costs. The burden on patients of lost productivity or lost wages and out-of-pocket costs is considerably less with a shorter regimen. From preliminary results of the STREAM Stage 1 trial, there was a documented reduction in costs to patients due to less time away from work, fewer clinic visits, and less spending on supplementary food (252).

\section{Harms}

In the STREAM Stage 1 clinical trial, an adverse event of grade 3 or higher occurred in $45.4 \%$ of participants in the long-regimen group and in $48.2 \%$ in the short-regimen group (8). Prolongation of either the QT interval or the QTc to 500 milliseconds occurred in $11.0 \%$ of participants in the short-regimen group, compared with $6.4 \%$ in the long-regimen group $(P=0.14)$. Death occurred in $8.5 \%$ of participants in the short-regimen group and in $6.4 \%$ in the long-regimen group, and acquired resistance to fluoroquinolones or aminoglycosides occurred in $3.3 \%$ and $2.3 \%$, respectively. Among the participants who had HIV coinfection at baseline, 18 of $103(17.5 \%)$ in the short-regimen group died, compared with 4 of $50(8.0 \%)$ in the 
PICO Question 19-Surgery for MDR-TB: Should elective lung resection surgery (i.e., lobectomy or pneumonectomy) be used as an adjunctive therapeutic option in combination with antimicrobial therapy, versus medical therapy alone, for adults with MDR-TB?

Recommendation 19a: We suggest elective partial lung resection (e.g., lobectomy or wedge resection), rather than medical therapy alone, for adults with MDR-TB receiving antimicrobial-based therapy (conditional recommendation, very low certainty in the evidence). The writing committee believes this option would be beneficial for patients for whom clinical judgement, supported by bacteriological and radiographic data, suggests a strong risk of treatment failure or relapse with medical therapy alone.

Recommendation 19b: We suggest medical therapy alone, rather than including elective total lung resection (pneumonectomy), for adults with MDR-TB receiving antimicrobial therapy (conditional recommendation, very low certainty in the evidence).

long-regimen group (HR in a post hoc analysis, 2.23; 95\% CI, 0.76-6.60) (8). Similarly, in a recently published IPDMA, a greater proportion of individuals who received the shorter regimen had less treatment success and more death (249). In the IPDMA, other adverse effects were also statistically insignificant with the shorter regimen, including deafness and ototoxicity (relative risk, 1.5; 95\% CI, 0.6-4.0), liver injury (relative risk, 2.2; 95\% CI, 0.5-10.3), hepatitis (relative risk, 2.5; 95\% CI, $0.3-21.2$ ), and renal impairment (relative risk, 4.5; 95\% CI, 0.6-35.2). One of the most concerning adverse effects of the shorter regimen is hearing loss, with $7.1 \%$ reported in the African study and between $0 \%$ and $23 \%$ in the meta-analysis (249,

250). The STREAM Stage 1 trial found that ear and labyrinth disorders occurred in $7.4 \%$ participants on the shorter regimen, compared with $5.7 \%$ with the longer regimen, which was not statistically significant (8); the frequency may have been lower than in cohort studies because hearing loss was not monitored by audiometry in STREAM Stage 1.

\section{Additional Considerations}

When applying the eligibility criteria from WHO for using the shorter-course, standardized regimen to the population included in our PS-matched IPDMA $(7,253),>15 \%$ of individuals would have been eligible for the standardized, shorter regimen. In Europe, patient eligibility for the shorter-course regimen has ranged from $7.9 \%$ ( 48 of 612 new cases) in a study performed at TB reference centers (254) to $16.9 \%$ in a surveillance-based study performed by the European Centre for Disease Prevention and Control (ECDC)

$(93,107,255)$. In the United States, $>15 \%$ of patients with MDR-TB would be eligible for the shorter-course MDR-TB regimen (256). Data from California showed either $14.6 \%$ or $20.5 \%$ eligibility, based on whether high-dose isoniazid or ethionamide would be determined effective on the basis of genetic kat $\mathrm{G}$ or inh $\mathrm{A}$ mutations, respectively (257). The availability of molecular and growth-based DST is key to determining the potential eligibility of shorter-course regimen use. The combined use of both modalities can better inform drug resistance and potential treatment regimens. High-dose isoniazid is likely to be active against organisms with low-level isoniazid resistance, commonly associated with a mutation in the inhA gene that also confers resistance to ethionamide (257). Ethionamide is more likely to be active against $M$. tuberculosis organisms with high-level resistance to isoniazid, associated with a mutation in kat $G$ and which are less commonly resistant to ethionamide (257). Global data have shown that $k a t G$ is present in $64.3 \%$ of the cases tested and inhA in $19.2 \%$, with some regional differences (258). The shorter regimen includes both ethionamide and high-dose isoniazid and therefore is likely to be effective against both of these common MDR-TB resistance patterns (257, 259). There is uncertainty regarding the diagnostic accuracy and reproducibility of ethambutol and pyrazinamide growthbased DST assays (260). For ethambutol, the ECDC has reported that the growth-based DST when performed on
Mycobacteria Growth Indicator Tube (MGIT) is reliable (107), whereas Model Performance Evaluation Program data have identified variability in detecting ethambutol resistance, with the majority of laboratories having disagreement on one to several strains with ethambutol resistance (261). Alternatively, molecular testing for pncA mutations as a method for determining pyrazinamide resistance may be more reliable, because on average $85 \%$ of TB strains resistant to pyrazinamide will have such a mutation $(217,221,222)$. In limited-resourced settings, testing all the drugs composing the shorter-course regimen may not be possible $(106,108)$. In contrast, in Europe, DST for ethambutol $(93,107)$ and pyrazinamide $(106)$ is well studied, but kat $G$ and inhA genetic mutations are not. In the United States, growth-based DST data are available for ethambutol, pyrazinamide, high- and lowlevel isoniazid, and ethionamide, and molecular testing is available through some state public health laboratories and through the CDC's MDDR service (257). Therefore, considering patients with either kat $G$ or inhA (but not both) mutations to be eligible for the shorter regimen might be rational. Last, the rate of cross-resistance between ofloxacin and moxifloxacin is likely not complete. Data from the ECDC showed that $81 \%$ of $M$. tuberculosis isolates that were resistant to ofloxacin were also resistant to moxifloxacin. However, other settings (e.g., Bangladesh and Pakistan) have shown cross-resistance as low as $7 \%$ (107).

\section{Conclusions}

The shorter-course regimen was judged by the guidelines committee to have minimal desirable effects (on treatment success, mortality, and culture conversions) and small to moderate undesirable effects (adverse events, limited applicability, and the use of kanamycin as part of the standardized regimen), and includes drugs for which there is documented or high likelihood of resistance (e.g., isoniazid, ethionamide, and pyrazinamide). Although the WHO's STREAM Stage 1 randomized trial found the shorter-course regimen to be noninferior to a long regimen with respect to the primary efficacy outcome (8), the guideline committee cannot make a recommendation either for or against this standardized shorter-course regimen compared 
PICO Question 20-Treatment of isoniazid-resistant TB: PICO Question 20a: Should patients with isoniazid-resistant TB be treated with a regimen composed of a fluoroquinolone, rifampin, ethambutol, and pyrazinamide for 6 months compared with rifampin, ethambutol, and pyrazinamide (without a fluoroquinolone) for 6 months?

PICO Question 20b: Should patients with isoniazid-resistant TB be treated with a regimen composed of fluoroquinolone, rifampin, and ethambutol for 6 months and pyrazinamide for the first 2 months compared with a regimen composed of a fluoroquinolone, rifampin, ethambutol, and pyrazinamide for 6 months?

Recommendation 20a: We suggest adding a later-generation fluoroquinolone to a 6-month regimen of daily rifampin, ethambutol, and pyrazinamide for patients with isoniazid-resistant TB (conditional recommendation, very low certainty in the evidence).

Recommendation 20b: In patients with isoniazid-resistant TB treated with a daily regimen of a later-generation fluoroquinolone, rifampin, ethambutol, and pyrazinamide, we suggest that the duration of pyrazinamide can be shortened to 2 months in selected situations (i.e., noncavitary and lower-burden disease or toxicity from pyrazinamide) (conditional recommendation, very low certainty in the evidence).

with longer individualized regimens. We instead make a research recommendation for the conduct of randomized clinical trials evaluating the efficacy, safety, and tolerability of modified shorter-course regimens that include newer oral agents, exclude injectables, and include drugs for which susceptibility is confirmed or deemed to be highly likely. If this shorter-course regimen is used, we recommend obtaining DST for all medications in the regimen, with the exception of clofazimine, for which reliable testing is not available, and recommend careful side effect monitoring, including high-quality audiometry, monthly microbiologic monitoring, and close case management, especially in persons with HIV.

\section{Research Needs}

We make a research recommendation for the conduct of randomized clinical trials evaluating the efficacy, safety, and tolerability of modified shorter-course regimens that include newer oral agents, exclude injectables, and include drugs for which susceptibility is confirmed or deemed to be highly likely. Further research is needed on medications such as linezolid, bedaquiline, and other medications currently in clinical trials as substitutions in the regimen if patients experience adverse effects or resistance develops to any of the medications in the regimen (262). Research on modified shorter-course regimens for pediatric patients and in individuals living with HIV is also needed. Until more data regarding the use and outcomes of the shorter-course regimens in patients with HIV are available, this treatment approach should preferentially be considered only within a research study. Current trials are underway to help answer some these questions (262).

\section{Role of Surgery in MDR-TB}

Surgery was one of the first therapeutic approaches for treating TB. It was replaced by chemotherapy between 1960 and 1975 . However, several scientific societies and national and international organizations suggest consideration of surgery as an adjunctive therapy for MDR-TB. This is based on the results of observational retrospective studies. Selected indications are highlighted, including failure of drug therapy, relapse, localized (e.g., an isolated cavity) or extensive pulmonary $\mathrm{TB}$, and clinical complications (e.g., hemoptysis or empyema) $(23,263-270)$.

\section{Summary of the Evidence}

Systematic reviews and meta-analyses have been performed on the role of surgery in patients with MDR- and XDR-TB $(263,271$, 272). The main limitation of systematic reviews of surgery in MDR and XDR-TB that summarize results of observational studies combining study-level data is the tremendous variability in patient characteristics, background chemotherapy regimens, and types of surgical procedures $(263,271,272)$. An IPDMA of surgery in MDR-TB was designed to address those shortcomings (271). Procedures and methodology to assemble and rank the certainty in the evidence are reported in APPENDIX A, with evidence profiles for PICO questions reported in ApPENDIX B.
The IPDMA identified 67 cohort studies, of which 45 could not be used because either individual patient data were not available or the surgical status of patients was not known. Twenty-six studies comprising 6,431 patients with MDR-TB were included (271). We used data from this IPDMA to generate the evidence profiles. Despite the analytic advantages of an IPDMA, which allows for adjustment on baseline imbalance in many prognostic factors, there was a substantial residual risk of bias in the results. There is no information about the impact of surgery on adverse effects or quality of life.

\section{Benefits}

Patients with partial lung resection had a higher probability of treatment success, as opposed to treatment failure, relapse, or death (aOR, 3.0; 95\% CI, 1.5-5.9). However, the estimate is very uncertain because of the limitations of individual studies and lack of precision in results.

\section{Harms}

In the published IPDMA, a substantially higher proportion of patients who had a pneumonectomy died (8.5\%) compared with those who had a partial resection (2.2\%), but the authors could not establish whether patients died as a result of surgical complications or of their TB (271). The estimates of the effects of pneumonectomy on risk of death (aOR, 1.8; 95\% CI, 0.6-5.1) and treatment success (aOR, 0.8; 95\% CI, 0.1-6.0) were not statistically significant. As for partial lung resection, both estimates are very uncertain. Treatment success in patients with XDR-TB was noted to be lower when patients underwent surgery compared with patients who did not (aOR, 
0.4; 95\% CI, 0.2-0.9), an effect that was heterogeneous across studies and may also be confounded by factors that predisposed to poor outcomes in these patients (271). Alternatively, patients who were healthy enough to withstand surgery may have intrinsically lower mortality, so it is difficult to predict the direction of potential bias.

\section{Additional Considerations}

In the MDR-TB treatment guidelines updated in 2016, WHO recommended elective partial surgery as an intervention complementary to chemotherapy in specifically selected individuals (23). The committee acknowledged there is significant uncertainty, with a paucity of publications and data regarding patient preferences, acceptability by surgical personnel, cost, and feasibility of performing elective surgery as an intervention for MDR-TB.

\section{Conclusions}

On the basis of the limited evidence that is available, there appears to be a net benefit from an elective partial lung resection (e.g., lobectomy or wedge resection) when offered together with a recommended MDR-TB regimen, compared with medical therapy alone. Committee members believed that this therapeutic option would probably be more beneficial when clinical judgement, supported by bacteriological and radiographic data, suggests a strong risk of relapse or treatment failure with medical regimen alone. We found no currently available evidence that pneumonectomy would be beneficial for patients with MDR TB receiving a background drug regimen.

\section{Research Needs}

Rigorously designed and conducted studies, ideally well-done randomized trials that measure and report benefits but also adverse effects and quality of life, are needed to clarify the role of surgery in the management of patients with MDR-TB. The following specific issues need to be addressed: the optimal timing for surgery, the optimal drug regimens and their duration before and after surgery, the role of surgery in special populations and patients with comorbid conditions (e.g., those living with HIV), the optimal surgical approaches, the optimal infection control measures to be implemented perioperatively, and the role of pulmonary rehabilitation (263, $267,270,273)$

\section{Treatment of Isoniazid- Resistant TB}

Isoniazid is an important first-line agent for the treatment of $\mathrm{TB}$, possessing potent early bactericidal activity against $M$. tuberculosis. However, monoresistance to isoniazid is frequent worldwide, with an estimated prevalence of $8 \%$ (range, $5-11 \%$ ) of TB cases (12). A recent systematic review and metaanalysis compared the treatment outcomes of isoniazid-resistant TB to outcomes of drugsusceptible TB and found that treatment of isoniazid-resistant TB with first-line drugs resulted in suboptimal outcomes, with higher treatment failure (11\% vs. $1 \%)$ and relapse (10\% vs. $5 \%)$ (274). In addition, the study found that standardized empirical treatment of new isoniazid-resistant TB cases may be contributing to higher rates of acquired drug resistance ( $8 \%$ vs. $0.3 \%$ ). Prior ATS, CDC, and IDSA guidelines recommended treatment with a standard four-drug regimen (isoniazid, rifampin, pyrazinamide, and ethambutol) for 6 months, with discontinuation of isoniazid after the results of DST are known and if isoniazid resistance was found (275).

\section{Summary of the Evidence}

Procedures and methodology to assemble and rank the certainty in the evidence are reported in APPENDIX A, with evidence profiles for PICO questions reported in ApPENDIX B. An IPDMA of 33 datasets with 6,424 patients, of whom 3,923 patients in 23 studies received regimens related to isoniazid-resistant $\mathrm{TB}$, was used as the evidence (276). Regimens of interest for our analyses (all with or without isoniazid) were 1) rifampin, ethambutol, and pyrazinamide; and 2) rifampin, ethambutol, and pyrazinamide, plus a fluoroquinolone. For these analyses, isoniazid-resistant TB was defined based on phenotypic resistance to isoniazid and susceptibility to rifampicin, with or without additional resistance to pyrazinamide, ethambutol, or streptomycin. Critical concentrations of $0.1 \mu \mathrm{g} / \mathrm{ml}$ or 0.2 $\mu \mathrm{g} / \mathrm{ml}$ were used in 21 out of 23 centers for the definition of resistance to isoniazid (276). For PICO Question 20a, we found few studies that used regimens of 6 months of rifampin, ethambutol, and pyrazinamide, plus a fluoroquinolone, but several that used regimens of 8 to 9 months of this regimen; hence, we combined 6 with $>6$ months for this PICO. For the comparator of rifampin, ethambutol, pyrazinamide with or without isoniazid, we similarly combined regimens of 6 and $>6$ months' duration regimens, after comparison between these durations showed outcomes were not significantly different. For PICO Question 20b, few patients received regimens that had a fluoroquinolone, rifampin, ethambutol, and a shorter duration of pyrazinamide. A total of 118 patients received 1 to 3 months of pyrazinamide in conjunction with 6 and $>6$ months' durations of rifampin, ethambutol, and fluoroquinolone-containing regimen and were available for analyses.

\section{Benefits}

Compared with 6 months of daily rifampin, ethambutol, and pyrazinamide (with or without isoniazid), adding a fluoroquinolone to this regimen was associated with significantly greater treatment success (aOR, 2.8; 95\% CI, 1.1-7.3) but with no significant effect on mortality (aOR, 0.7; 95\% CI, 0.4-1.1) or acquired rifampicin resistance (aOR, 0.1; 95\% CI, 0.0-1.2). When evaluating the impact of shortening the duration of pyrazinamide (ranging from 1-3 mo) in a regimen that contains a fluoroquinolone, the treatment success was very high, with 117 of 118 patients achieving treatment success. On the other hand, comparisons of shorter pyrazinamide regimens to regimens including both a fluoroquinolone and pyrazinamide for $\geqslant 6$ months did not show significantly different results (aOR, 5.2; 95\% CI, 0.6-46.7). Similar results were obtained when the comparisons were restricted to patients receiving latergeneration fluoroquinolones, namely moxifloxacin, levofloxacin, and gatifloxacin (data not shown).

\section{Harms}

The outcome of adverse events from TB drugs was intended to be assessed in the IPDMA but could not be analyzed because these outcomes were either not reported or reported with very different definitions. The adverse events associated with TB drugs, especially pyrazinamide, are well established $(227,277)$.

\section{Additional Considerations}

We note that the estimates of effect were imprecise from the IPDMA because of the small number of patients who received the 
Table 11. Select Antiretroviral and Non-Rifamycin-based Antituberculosis Drug Overlapping Toxicities and Potential Adverse Drug-Drug Interactions

\section{Potential Overlapping Toxicities and} Drug-Drug Interactions

Antiretroviral Drugs

Lopinavir/ritonavir, efavirenz

Note PR interval prolongation ${ }^{*}$ with atazanavir, lopinavir/ritonavir

Hepatic cytochrome P450 enzyme system metabolism

Nephrotoxicity

Mental health changes (depression, psychosis, dizziness, etc.)

Peripheral neuropathy

Hepatotoxicity

Skin rash

Dysglycemia

Myelosuppression/cytopenias

Lactic acidosis
Induce CYP P450 metabolism: efavirenz, etravirine, and nevirapine

Impede CYP P450 metabolism: protease inhibitors, cobicistat

Tenofovir, ${ }^{\ddagger}$ atazanavir

Isolated creatinine elevation ${ }^{\S}$ : cobicistat, dolutegravir

Efavirenz, rilpivirine; dolutegravir, elvitegravir, raltegravir

Stavudine, zidovudine

Lactic acidosis with hepatic steatosis higher risk with stavudine, zidovudine; protease inhibitors; nevirapine (higher risk in patients with elevated CD4 cell counts); less common with efavirenz, etravirine and rilpivirine; maraviroc

Indirect hyperbilirubinemia ${ }^{\|}$: atazanavir

Nevirapine (higher risk in patients with elevated CD4 cell counts), efavirenz, etravirine, rilpivirine. Any protease inhibitor (especially those containing sulfonamide moiety: e.g., darunavir); abacavir (hypersensitive reaction a risk in patient who are HLA-B5701 positive); raltegravir

Lopinavir/ritonavir, ritonavir, stavudine, zidovudine

Zidovudine

Stavudine, zidovudine

\section{Non-Rifamycin TB Drugs}

Fluoroquinolones $^{\dagger}$, bedaquiline, delamanid, clofazimine

Bedaquiline, delamanid

Aminoglycosides, capreomycin

Cycloserine, isoniazid, ethionamide, fluoroquinolones $^{\dagger}$

Aminoglycosides, capreomycin, linezolid, isoniazid, ethionamide, cycloserine, fluoroquinolones ${ }^{\dagger}$

Isoniazid, pyrazinamide, ethionamide, $p$-aminosalicylic acid, clofazimine

All TB drugs

Note skin pigmentation with clofazimine use

Thioacetazone should be avoided in people with HIV, because of an elevated risk of a severe adverse skin reaction

Ethionamide, $p$-aminosalicylic acid, fluoroquinolones, linezolid

Linezolid

Linezolid

Definition of abbreviation: TB = tuberculosis.

Saquinavir, indinavir, fosamprenavir, tipranavir, and didanosine are older antiretroviral drugs that are rarely used in the United States. They do have many of the adverse interactions listed above with select TB drugs, and clinicians considering the use of these agents should therefore consult with an expert.

*Use with caution in patients with underlying cardiac dysrhythmia.

${ }^{\dagger}$ Fluoroquinolones include ciprofloxacin, ofloxacin, levofloxacin, gatifloxacin, and moxifloxacin.

¥Tenofovir alafenamide is a prodrug of tenofovir and U.S. Food and Drug Administration approved in 2015. It is associated with decreased incidence of osteoporosis and nephrotoxicity compared with tenofovir disoproxil fumarate through achieving higher intracellular drug concentrations with a lower dose administered.

$\S$ Increases in serum creatinine via decrease in renal tubular creatinine excretion are commonly seen with cobicistat and dolutegravir usage. This is not a toxicity.

"Indirect hyperbilirubinemia is expected with atazanavir and indinavir. This is not a toxicity.

regimens of interest. Given that pyrazinamide is the most toxic of the present first-line drugs, a key potential advantage of adding a fluoroquinolone would be to shorten the duration of pyrazinamide to the initial 2 months of treatment. Although the IPDMA only had 118 patients receiving fluoroquinolone-containing regimens with shorter durations of pyrazinamide, it is noted that treatment success was very high in this group (117 of 118). On the basis of the efficacy signals seen and the known toxicities of prolonged pyrazinamide, the committee viewed the balance of benefits and harms to favor shortening the duration of 
pyrazinamide when a later-generation fluoroquinolone is included in the regimen in patients in whom there is toxicity anticipated or experienced because of pyrazinamide or when the patient has noncavitary, lower burden of disease. Finally, plasma peak concentration and exposure to moxifloxacin have been shown to decrease by approximately $30 \%$ when coadministered with rifampin $(278,279)$. The impact of these decreased exposures on outcomes has not been established. However, some experts opt to use levofloxacin when combined with rifampin.

\section{Conclusions}

We conclude that in patients with isoniazidresistant $\mathrm{TB}$, the addition of a later-generation fluoroquinolone to 6 months of daily rifampin, ethambutol, and pyrazinamide improves treatment success rates. In patients in whom toxicity from pyrazinamide is anticipated or experienced, or in patients with active TB with lower burden of disease (i.e., noncavitary), the committee viewed the balance of benefits and harms to favor shortening the duration of pyrazinamide when a later-generation fluoroquinolone is included in the regimen, acknowledging that the certainty in the evidence is very low and more research is needed.

\section{Research Needs}

All but 2 of the 23 studies included in the IPDMA were observational. Moreover, the analyzed population included only 37 children, 119 patients with diabetes, and 249 patients with HIV infection. Given the burden of isoniazid-resistant TB worldwide, clinical trials that include these special populations and evaluate new regimens, including the evaluation of the efficacy, safety, and tolerability of shorter versus longer durations of pyrazinamide, are urgently needed.

\section{Treatment of MDR-TB in Special Situations}

\section{HIV Infection}

Among patients with HIV and TB, rifampin resistance has been identified more often compared with those without HIV $(280,281)$. Patients with MDR-TB and HIV have up to a fourfold higher risk of mortality compared with patients with MDR-TB without HIV (282). Low CD4 cell counts (e.g., $\leqslant 50$ cells/ $\mu \mathrm{l})$ in patients with HIV and MDR-TB further correlate with higher mortality (283, 284). Numerous practice guidelines recommend HIV testing of people with suspected or confirmed TB $(11,20,34,285)$, regardless of drug resistance. Because of their high risk for mortality early in the course of TB disease, people with HIV in whom TB is suspected are recommended to receive rapid testing for TB using nucleic acid amplification tests coupled with molecular diagnostic DST for rifampin (with or without isoniazid) $(13,20)$.

For patients with HIV receiving therapy for drug-susceptible TB, studies found significantly lower mortality among patients receiving concurrent antiretroviral therapy (ART) compared with those not receiving ART (286-288). U.S. practice guidelines recommend starting ART within 2 weeks of initiating treatment for drug-susceptible non-CNS TB for patients with CD4 cell counts $<50$ cells/ $\mu$ l and by 8 to 12 weeks for patients with higher CD4 cell counts (11, 289). Although the optimal timing for starting ART to reduce patient mortality has not yet been adequately determined for patients with MDR-TB, lower mortality rates have been shown in multiple studies among patients with MDR-TB receiving concurrent ART compared with those not receiving ART (283, 290-297), especially among patients with TB with CD4 counts $<50$ cells/ $\mu l(283,292)$. The decreased mortality

PICO Question 21-Treatment of Contacts Exposed to MDR-TB: Should contacts exposed to an infectious patient with MDR-TB be offered LTBI treatment versus followed with observation alone?

Recommendation 21: For contacts with presumed MDR LTBI due to exposure to an infectious patient with MDR-TB, we suggest offering treatment for LTBI (conditional recommendation, very low certainty in the evidence). We suggest 6 to 12 months of treatment with a later-generation fluoroquinolone alone or with a second drug, on the basis of drug susceptibility of the source-case M. tuberculosis isolate. On the basis of evidence of increased toxicity, adverse events, and discontinuations, pyrazinamide should not be routinely used as the second drug.

seen in patients treated for MDR-TB who concurrently receive ART, especially among those with CD 4 cell counts $<50$ cells $/ \mu l$, supports a similar ART management approach as recommended for drugsusceptible TB.

MDR-TB of the CNS in patients with HIV presents additional challenges. TB meningitis with isoniazid-resistant $\mathrm{TB}, \mathrm{RR}$ TB, or MDR-TB can be associated with higher mortality compared with drugsusceptible disease (298-300). Starting ART early in patients with TB is associated with a higher incidence of immune reconstitution inflammatory syndrome $(286,288,301)$, and this can be even more problematic in CNS disease. A recent study in Vietnam of patients with advanced AIDS found a higher incidence of potentially lifethreatening (grade 4) adverse events among patients with TB meningitis starting ART early compared with those delaying ART until after 2 months of standardized firstline TB treatment and did not show a survival benefit of starting ART early (302). It has been recommended to delay starting ART by 8 weeks in patients with CNS TB and HIV (11); however, there is a paucity of data in patients with MDR-TB of the CNS. The optimal approach for initiation of ART in patients with this medical condition remains uncertain, and close clinical monitoring is warranted.

Drug interactions between antiretroviral and anti-TB agents are common in the management of patients with HIV and TB, particularly with rifamycins. Because the rifamycins (with the possible exception of rifabutin) are not used for treatment of MDR-TB, interactions of other TB medication classes with ART should be considered. Bedaquiline and/or delamanid might be considered for use in patients with HIV. Although efavirenz can produce a decrease in serum bedaquiline concentrations and this combination is avoided, other ART drugs, including the protease inhibitors and cobicistat, can result in increased serum bedaquiline levels. The current WHO recommendations for the use of delamanid apply to patients living with HIV (7). Drug-drug interaction studies in healthy volunteers of delamanid with tenofovir, efavirenz, and lopinavir/ritonavir show that no dose adjustments were needed (303). Nonetheless, when delamanid is included in the regimen for MDR-TB in HIVinfected patients, the design of their 
ART regimens should be developed in consultation with HIV and ART experts. A thorough review of all patients' medications should be performed, in consultation with MDR-TB experts, to select individualized regimens with less potential for overlapping ART/TB drug toxicities (Table 11). Useful webpages regarding drug interactions (TB/HIV and other) are available from AIDSinfo (https://aidsinfo.nih.gov/guidelines), CDC (http://www.cdc.gov/tb/publications/ guidelines/TB_HIV_Drugs/default.htm), University of California San Francisco (http:// hivinsite.ucsf.edu/insite?page $=$ ar-00-02), University of Liverpool (http://www.hivdruginteractions.org/), and Indiana University (http://medicine.iupui.edu/ clinpharm/ddis/).

The management of MDR-TB is more complex among patients with HIV infection. The higher pill burden of combined ART with expanded TB drug therapy, potential drug-drug interactions, the management of immune reconstitution inflammatory syndrome, and other concurrent HIV-associated opportunistic diseases all pose unique challenges in the care of these patients. The management of patients with HIV and MDR-TB can best be performed by a multidisciplinary care team composed of health providers experienced in MDR-TB, HIV, and public health case management $(304,305)$. MDR-TB experts can be found through CDC-sponsored TB Centers of Excellence for Training, Education, and Medical Consultation (http://www.cdc.gov/ tb/education/rtmc/default.htm), national, state and local TB control programs (https://www.cdc.gov/tb/links/tboffices.htm), and international MDR-TB expert groups such as the Global TB Network (6).

\section{Children}

On the basis of recent modeling studies, it is estimated that there are about 1 million incident cases of TB in children annually and 230,000 deaths caused by the disease $(306,307)$. About 35,000 cases of MDR- and XDR-TB occur in children annually (308, 309). Our PS-matched IPDMA did not include sufficient numbers of children to allow the formulations of GRADE-based recommendations. Nonetheless, on the basis of a recent IPDMA of 975 children with MDR-TB from 18 countries, recent pharmacokinetic studies in children, and several observational studies showing good outcomes, the recommendations noted on choice of drugs, composition of regimens, and durations of treatment for adults can also be applied to children with MDR-TB $(113,119,124,161,165,170,200,310)$.

There are some special considerations for formulating effective regimens for children of various ages and adolescents. The bacterial burden in young children with TB is much smaller than that in most adults with TB. As a result, most drug resistance in children was present when the organism was inhaled (primary resistance), and further development of resistance while on therapy (secondary resistance) is much less common in children. However, the paucibacillary nature of childhood TB also makes microbiologic confirmation much more difficult. The only way to determine drug susceptibility in cases that meet clinical definitions of TB disease (i.e., microbiologic confirmation is not available) is by linking the child to a specific source case for whom the drug susceptibilities of the organism are known. Linking the child and a specific case is more feasible in lowburden settings to which these guidelines apply but can be difficult in high-burden settings when there may be more than one possible source case. Also, standard definitions of relapse and treatment failure for pediatric $\mathrm{TB}$ trials are inconsistent because the low burden of organisms in children makes microbiologic confirmation of these outcomes difficult.

There are several technical factors that can affect the outcome of treatment for MDR- and XDR-TB in children. The two age extremes of childhood have been somewhat neglected in studies of MDR- and XDR-TB. Little is known about the pharmacokinetics, safety, and tolerability of the drugs used to treat drug-resistant TB in neonates, infants, and toddlers (115). Children, especially those $<2$ years of age, are more prone to developing disseminated $\mathrm{TB}$, including meningitis. Drugs that penetrate well into the CSF, such as linezolid, might have an advantage over drugs that appear to have less penetration, such as ethambutol and bedaquiline.

Adolescents can develop TB similar to that found either in adults or in young children. However, adolescent patients often have been excluded from TB treatment trials. Most of the oral drugs used to treat MDRand XDR-TB are not licensed for children. Although the Global Drug Facility has pediatric dispersible tablets available (http://stoptb.org/gdf/drugsupply/drugs_ available.asp), these formulations are not registered with FDA or the European Medicines Agency, which has limited commercial availability of child-friendly dosage forms. As a result, for younger children, the medication often has to be crushed or put into suspension or capsules once opened, and the pharmacokinetics and pharmacodynamics of these various preparations are unknown. HIV-infected children may be exposed to lower concentrations of certain orally administered drugs than HIV-uninfected children given the same bodyweight dose. Unfortunately, the pharmacokinetics in HIV-infected children of drugs used to treat MDR- and XDR-TB are largely unstudied. Children generally have a more difficult time tolerating injectable medications because of pain and the fact that many children with TB are malnourished and have diminished muscle mass. With the recent development of new oral drugs, it is hoped that injectable drugs can be avoided in children whenever possible. Fortunately, children generally tolerate the oral TB drugs better than adults, with fewer serious adverse events resulting in fewer breaks in therapy. Also, most children with TB have not developed the common chronic diseases of adulthood and will not suffer complications of them during treatment. However, drug adverse effects can be difficult to assess in children and likely are underreported. In general, the same schedules used to monitor adverse events and laboratory abnormalities in adult patients treated for MDR- or XDR-TB also should be used for children.

Outcomes of MDR-TB treatment in children. A recently published systematic review (33 studies) and IPDMA (28 of the studies) described treatment outcomes for 975 children with MDR-TB using random effects multivariate logistical regression adjusted for age, sex, HIV infection, malnutrition, severe extrapulmonary disease, and severe pulmonary disease on chest radiograph (113). Overall, 78\% had a successful treatment outcome, including $75 \%$ of the microbiologically confirmed cases. However, treatment was successful in only $56 \%$ of HIV-infected children who did not also receive ART during TB treatment compared with an $82 \%$ success rate in those also treated with ART. In children with confirmed MDR-TB, the use of injectable agents and high-dose isoniazid was associated with treatment success. Unfortunately, limitations of this study included that the vast majority of patients 
came from one site (Cape Town, South Africa), difficulty in estimating the treatment effects of individual drugs within multidrug regimens, the availability of only observational cohort studies, and that treatment decisions were based on the clinician's perception of illness, with resulting potential for bias.

Conclusions. Excellent treatment outcomes have been demonstrated in both trials and extensive clinical experience for children with MDR- and XDR-TB using individualized treatment regimens with the currently available drugs. Microbiologic cure and probable cure rates in children can reach $80 \%$ to $90 \%$ with early recognition of drug resistance and adequate treatment (311). The greatest difficulties have been recognizing that the child has MDR-TB and the ability of the child to tolerate injectable drugs; fortunately, with expanded knowledge of and experience with the newer oral drugs, such as bedaquiline and delamanid, pediatricians with expertise in MDR-TB believe that the majority of children with MDR-TB likely can be cured with an all-oral drug regimen.

\section{Pregnant Women}

Untreated MDR-TB during pregnancy can be associated with adverse maternal and fetal outcomes. Crucial gaps exist in the literature on treatment of MDR-TB in pregnant women, including the effectiveness, safety, and tolerability of available treatment regimens, as well as timing and duration of second-line drugs. In support of these guidelines, we conducted a systematic literature review with a focus on pregnant women with MDR-TB and included all original research reporting MDR-TB treatment outcomes during pregnancy, including case reports and case series, in all languages. We excluded animal studies, review articles, letters to the editor, and articles without documentation of MDR-TB treatment during pregnancy. Fulltext review and data extraction were conducted by three reviewers. Our initial search yielded 280 publications, of which 16 met inclusion criteria for full-text review (312-327); however, 3 publications were eventually excluded because of lack of data on medications or outcomes $(315,325$, 327). The remaining 13 articles were observational case reviews without any comparison groups. Treatment regimens reported were individualized according to drug susceptibility and tolerability of drugs.
Summary of the evidence. Of the 65 pregnant women for whom MDR-TB treatment outcome data were available, $49 \%$ $(n=32)$ were cured and $20 \%(n=13)$ completed treatment for a treatment success proportion of $69 \%$. Fourteen percent $(n=9)$ of the women died. Treatment failure was reported in $9 \%$ $(n=6)$, and $3 \%(n=2)$ were lost to followup. Across these studies, four women were still receiving treatment at the time of publication. Fetal outcomes included $78.5 \%$ ( $n=51)$ healthy births, with eight children being born premature or having low birth weight. Medical abortions were obtained by $12 \%$ of the patients $(n=8)$, and $3 \%(n=2)$ underwent spontaneous abortions. Stillbirth was reported for one child $(1.5 \%), 3 \%(n=2)$ of children were born with HIV, and 1.5\% $(n=1)$ had TB/HIV coinfection.

Conclusions. On the basis of the limited data available, we conclude that there is evidence to support treatment of MDR-TB during pregnancy, including the prescription of second-line drugs. Most of the second-line drugs are pregnancy category C per the U.S Food and Drug Administration, with the exception of bedaquiline and meropenem, which are classified as category B (according to the previous FDA letter-based classification system, currently undergoing revision [328]), and aminoglycosides, which are category D $(71,320,329)$. Despite low cure rates reported in the literature, we believe that the benefits of treatment to mother, child, and the community outweigh the harms. There is no evidence to support one particular regimen; however, most MDR-TB experts avoid aminoglycosides and ethionamide in pregnant women if alternative agents can be used for an effective treatment regimen.

Research needs. Global registries aimed at collecting data on the efficacy, safety, and tolerability, and maternal and fetal outcomes associated with MDR-TB regimens in pregnant women are urgently needed. Furthermore, given the significant morbidity and mortality associated with MDR-TB, a reconsideration of the current approach of assumed exclusion of pregnant women from MDR-TB clinical trials is warranted. A recent consensus statement from an international expert panel advocated for allowing pregnant and lactating women to remain eligible for phase III MDR-TB trials, unless there is a compelling reason for exclusion (330).

\section{Treatment of Contacts Exposed to MDR-TB}

In 1992 and 2000, ATS and CDC advised: 1) no treatment for MDR LTBI for persons not at high risk for progression to $\mathrm{TB}$ disease, but provision of clinical follow-up for TB disease signs and symptoms; 2) 6 to 12 months of treatment with two or more medications to which the isolate of the source case is susceptible $(331,332)$.

\section{Summary of the Evidence}

Procedures and methodology to assemble and rank the certainty in the evidence are reported in APPENDIX A, with evidence profiles for PICO questions reported in APPENDIX B. A systematic review of 21 published observational studies that examined outcomes of TB incidence, treatment completion, adverse effects, and cost effectiveness was used as the evidence (333). Six articles compared TB incidence among contacts receiving MDR LTBI treatment versus untreated contacts: 10 presented TB incidence only for contacts who received MDR LTBI treatment, and 5 presented TB incidence only for untreated contacts.

\section{Benefits}

Using data from five non-registry-matched comparison studies included in the systematic review of 21 published observational studies, MDR-TB incidence occurred in 2 of 190 (1.1\%) patients treated for MDR LTBI, compared with 18 of 126 $(14.3 \%)$ in those who received no MDR LTBI treatment (290). The estimated MDRTB incidence reduction was $90 \%$ (9-99\%), using a negative binomial model controlling for person time and overdispersion (333).

\section{Harms}

From 11 studies having data by regimen on treatment discontinuation due to adverse effects, there was high (51\%) treatment discontinuation among patients taking pyrazinamide-containing regimens (333). About one-third of patients taking fluoroquinolone-containing regimens, without pyrazinamide, had adverse effects, but only $2 \%$ discontinued treatment. For children $\leqslant 15$ years of age regardless of regimen, treatment discontinuation due to adverse effects was substantially less ( $5 \%$ vs. 33\%) (333). 


\section{Additional Considerations}

Modeled cost-effectiveness studies have shown greatest benefit using a fluoroquinolone-based MDR LTBI treatment. In one study, the most costeffective regimen was

fluoroquinolone/ethambutol, followed by fluoroquinolone alone, then by pyrazinamide/ethambutol (333). A pyrazinamide/fluoroquinolone regimen was particularly toxic, as measured by treatment discontinuation, and prevented about half as many TB cases as the most cost-effective option (333). An expert panel generated a policy brief on this topic recently, acknowledged that further evidence is urgently needed, but still endorsed the immediate implementation of postexposure management of household contacts of MDR-TB, endorsing the approach as being effective, feasible, and cost efficient (334). The Curry International TB Center Drug Resistant TB: Clinician's Survival Guide suggests an LTBI regimen of levofloxacin or moxifloxacin alone or combined with a second medication to which the isolate of the infectious source patient is susceptible (16).

Among those with presumed MDR LTBI, there is probable effectiveness of preventing TB through MDR LTBI treatment. From systematic reviews of studies reporting incidence for both treated and untreated contacts, TB incidence was significantly lower with MDR LTBI treatment $(333,335-339)$. In studies that published outcomes by regimen, there were high treatment discontinuation rates due to adverse effects and toxicity in persons taking pyrazinamide-containing MDRLTBI regimens (336, 337, 339-342). There were low treatment discontinuation rates due to adverse effects and toxicity in persons taking fluoroquinolone-containing MDR-LTBI regimens. Finally, children are at high risk for developing MDR-TB if infected with MDR LTBI and generally experience fewer adverse effects than adults. Given the uncertainties above, many TB experts and programs provide extended post-treatment completion follow-up for patients treated for presumed MDR LTBI.

\section{Conclusions}

For contacts with presumed MDR LTBI due to exposure to an infectious patient with MDR-TB, we suggest offering treatment for LTBI versus following with observation alone. For treatment of MDR LTBI, we suggest 6 to 12 months' treatment with a fluoroquinolone alone or with a second drug, on the basis of source-case isolate DST. On the basis of evidence of increased toxicity, adverse events, and discontinuations, pyrazinamide should not be routinely used as the second drug. In lieu of fluoroquinolone-based treatment, there are few data for the use of other secondline medications and, because of toxicity, they are not recommended by experts. For contacts to fluoroquinolone-resistant, pre-XDR-TB, pyrazinamide/ethambutol may be an effective option, if source-case isolate DST shows susceptibility to these drugs. In children, TB drugs are generally better tolerated, and levofloxacin is preferred because of the availability of an oral suspension formulation.

\section{Research Needs}

Randomized clinical trials on the effectiveness of safer, shorter, more-effective MDR LTBI regimens are underway. Three randomized clinical trials of MDR LTBI treatment for contacts to persons with infectious MDR-TB include: 1) TB-CHAMP (Tuberculosis Child Multidrug-Resistant Preventive Therapy Trial): 24 weeks of levofloxacin versus placebo in children $<5$ years of age, 2) V QUIN (Levofloxacin versus Placebo for the Treatment of Latent Tuberculosis among Contacts of Patients with MultidrugResistant Tuberculosis [The VQUIN MDR Trial]): 6 months of daily levofloxacin versus placebo, and 3) PHOENIx (Protecting Households on Exposure to Newly Diagnosed Index Multidrug-Resistant Tuberculosis Patients [PHOENIx MDRTB]): 6 months of daily delamanid versus 6 months of isoniazid. In addition, more information is needed on the cost effectiveness of treating contacts with presumed DR-TB infection using these newer MDR LTBI regimens.

\section{Summary of Key Differences between ATS/CDC/ERS/IDSA and WHO 2019 Consolidated Guidelines on Drug-Resistant Tuberculosis Treatment}

The 2019 WHO Consolidated Guidelines on Drug-Resistant Tuberculosis Treatment guidelines were based on a modified set of data that expanded on the initial individual patient dataset used for these
ATS/CDC/ERS/IDSA guidelines (3). For the 2019 WHO update, data from 625 patients were included by WHO from eight other datasets received in 2018 (Australia, Belarus, Brazil, France, Latvia, Republic of Korea, Russian Federation, and the EndTB project), as well as a sample of 3,626 patients receiving treatment in South Africa, including 1,210 cases started on bedaquiline in 2015. With these newer data added, the WHO individual patient dataset removed 3,367 records because of incomplete DST documentation (7). Despite the changes in the datasets described, WHO and ATS/CDC/ERS/IDSA recommendations are largely concordant, as they were derived concurrently, using a similar approach (GRADE methodology and a multidisciplinary Guideline Development Group), and were informed by an individual patient data meta-analysis that overlapped substantially. The main differences are highlighted below.

- WHO recommendations are stated as applying to MDR and RR-TB. Our ATS/CDC/ERS/IDSA guidelines do not address management of rifampin resistance in the absence of isoniazid resistance.

- WHO recommendations only retain the intensive/continuation phase denomination for regimens that incorporate amikacin and streptomycin. We anticipate that use of regimens that incorporate injectables will decline over time, and it is likely that both WHO and ATS/CDC/ERS/IDSA recommendations on treatment duration will change in the near future and the distinction between an initial and continuation phase will be reduced further in the context of newer, more potent all-oral regimens emerging.

- The duration of the injectable phase in the WHO recommendations relates to its total length (6-7 mo in 2019 update) rather than time after culture conversion as in these ATS/CDC/ERS/IDSA guidelines. The recommendation on total duration is similar in length in both guidelines. For the WHO guidelines, no separate recommendations are made for pre-XDR or XDR-TB.

- The minimum number of medicines likely to be effective at the start of 
treatment is five in the ATS/CDC/ ERS/IDSA guidelines and four in the WHO recommendations. The WHO recommendation was based on analyses that showed no clear benefit from exceeding four effective drugs at the start of treatment. The difference between WHO and ATS/CDC/ERS/IDSA guidelines may be due to the greater use of bedaquiline and other effective drugs in the latter updates of the IPDMA used by WHO. The ATS/CDC/ERS/IDSA recommendation chose five medicines on the basis of benefits noted in analyses shown in Tables 3 and 4 and also to anticipate toxicities related to MDR-TB drugs, wherein one or more agents would likely need to be held or permanently discontinued within the first 6 months of treatment.

- The WHO recommendations for linezolid and bedaquiline were strong, with the evidence judged to be of moderate certainty by the WHO Guideline Development Group, whereas the ATS/CDC/ERS/IDSA guidelines judged the certainty of the IPDMA to be of lower certainty. The WHO recommendation on delamanid was primarily determined by the outcomes of Trial 213 reported in aggregate format by the study investigator (a subsequent IPDMA of trial data did not change the strength of the recommendation or the positioning of the drug in terms of priority for choice in a longer regimen). The ATS/CDC/ERS/IDSA committee concurs with the WHO recommendation that delamanid can be used for treatment of MDR-TB.

- With respect to composing a regimen, Table 10 in these guidelines, providing a stepwise selection of agents, is different from the WHO guidance, which proposes three groupings, essentially combining steps. On the basis of judgements of the respective guideline committee groups, the ordering of drugs within groups is minimally different.

- WHO maintains the recommendation on the standardized 9- to 11-month shortercourse regimen, under specific conditions, whereas the ATS/CDC/ERS/IDSA committee, on the basis of the fact that the regimen is standardized with the use of drugs for which there is documented or high likelihood of resistance (e.g., isoniazid, ethionamide, pyrazinamide), could not make a recommendation for or against the shorter regimen compared with individualized regimens. Instead, the ATS/CDC/ERS/IDSA committee makes a research recommendation for the conduct of randomized clinical trials evaluating the efficacy, safety, and tolerability of modified shorter-course regimens that include newer oral agents, exclude injectables, and include drugs for which susceptibility is confirmed or highly likely.

- For patients with isoniazid-resistant TB, the $\mathrm{WHO}$ recommendations do not provide for a reduction of duration of pyrazinamide use under situations such as recommended by the ATS/CDC/ERS/IDSA guidance.

- Finally, although the WHO recommends evaluation of drug resistance, their guidance accepts that globally empirical regimens will continue to be used. The ATS/CDC/ERS/IDSA guidance requires microbiological data to create a regimen suitable for the individual patient's strain of tuberculosis.

This official clinical practice guideline was prepared by an ad hoc subcommittee of the ATS, CDC, ERS, and IDSA.

\section{Members of the subcommittee are as follows:}

Payam Nahid, M.D., M.P.H. ${ }^{1}$ (Co-Chair)

Giovanni Battista Migliori, M.D. ${ }^{2}$

(Co-Chair)

Giovanni Sotgiu, M.D., Ph.D. ${ }^{3 \S}$

(Co-Chair)

Terence Chorba, M.D., D.Sc., M.P.H. ${ }^{4}$ (Co-Chair)

Sundari R. Mase, M.D., M.P.H. ${ }^{4 \star}$ (Co-Chair) Barbara Seaworth, M.D. ${ }^{5}$ (Co-Chair)

Graham H. Bothamley, M.D., M.A., Ph.D. ${ }^{6}$

Jan L. BrozeK, M.D., Ph.D. ${ }^{7 \ddagger}$

Adithya Cattamanchi, M.D., M.A.S. ${ }^{1}$

J. Peter Cegielski, M.D., M.P.H. ${ }^{4}$

Lisa Chen, M.D. ${ }^{1}$

Charles L. Daley, M.D. ${ }^{8}$

Tracy L. Dalton, Ph.D. ${ }^{4}$

Raquel Duarte, M.D., Ph.D., M.P.H. ${ }^{9,10}$

Federica Fregonese, M.D. ${ }^{11}$

C. Robert Horsburgh, JR., M.D. ${ }^{12}$

Faiz Ahmad Khan, M.D.C.M., M.P.H. ${ }^{13}$

FAYEZ KHEIR, M.D., M.S.C.R. ${ }^{14 \S}$

ZHIYI LAN, M.Sc. ${ }^{13}$
Alfred Lardizabal, M.D. ${ }^{15}$

Michael Lauzardo, M.D. ${ }^{16}$

Joan M. Mangan, Ph.D., M.S.T. ${ }^{4}$

SuZANne M. Marks, M.P.H., M.A. ${ }^{4}$

Lindsay McKenNa, M.P.H. ${ }^{17}$

Dick Menzies, M.D. ${ }^{13}$

Carole D. Mitnick, Sc.D. ${ }^{18}$

Diana M. Nilsen, M.D. ${ }^{19}$

Farah Parvez, M.D., M.P.H. ${ }^{4,19}$

Charles A. Peloquin, Pharm.D. ${ }^{16}$

AnN Raftery R.N., P.H.N., M.S. ${ }^{1}$

H. Simon SchaAf, M.D. ${ }^{20}$

Neha S. Shah, M.D., M.P.H. ${ }^{4 \|}$

JeFFrey R. STARKe, M.D. ${ }^{21}$

John W. Wilson, M.D. ${ }^{22}$

Jonathan M. WORTham, M.D. ${ }^{4}$

*Present address: World Health Organization, Southeast Asian Regional Office, New Delhi, India.

${ }^{\ddagger}$ Lead methodologist.

$\$$ Methodologist.

"Present address: Division of AIDS, National Institute of Allergy and Infectious Diseases, National Institutes of Health, Bethesda, Maryland.
${ }^{1}$ University of California San Francisco, San Francisco, California; ${ }^{2}$ Istituti Clinici Scientifici Maugeri IRCCS, Tradate, Italy; ${ }^{3}$ University of Sassari, Sassari, Italy; ${ }^{4}$ Centers for Disease Control and Prevention, Atlanta, Georgia; ${ }^{5}$ University of Texas Health Science Center, Tyler, Texas; ${ }^{6}$ Queen Mary University of London and London School of Hygiene and Tropical Medicine, London, United Kingdom; ${ }^{7}$ McMaster University, Hamilton, Ontario, Canada; ${ }^{8}$ National Jewish Health, Denver, Colorado; ${ }^{9}$ Instituto de Saúde Pública, Porto, Portugal; ${ }^{10}$ Centro Hospitalar de Vila Nova de Gaia/Espinho, Vila Nova de Gaia, Portugal; ${ }^{11}$ Université de Montréal, Montreal, Quebec, Canada; ${ }^{12}$ Boston University, Boston, Massachusetts; ${ }^{13}$ McGill University, Montreal, Quebec, Canada;

${ }^{14}$ Tulane University Health Sciences Center, New Orleans, Louisiana; ${ }^{15}$ New Jersey Medical School, Newark, New Jersey;

${ }^{16}$ University of Florida, Gainesville, Florida;

${ }^{17}$ Treatment Action Group, New York, New York;

${ }^{18}$ Harvard Medical School, Boston,

Massachusetts; ${ }^{19} \mathrm{New}$ York City Department of Health and Mental Hygiene, New York, New York; ${ }^{20}$ Stellenbosch University, Cape Town, South Africa; ${ }^{21}$ Baylor College of Medicine, Houston, Texas; and ${ }^{22}$ Mayo Clinic, Rochester, Minnesota 
Author Disclosures: G.H.B. served as a speaker for Johnson \& Johnson. C.L.D. served on an advisory committee for Insmed, Otsuka Pharmaceutical, Horizon, Johnson \& Johnson, Paraek, and Spero; served on a data and safety monitoring board for Otsuka Pharmaceutical and Sanofi; and received research support from Insmed. C.R.H. served on a data and safety monitoring board for Janssen. F.A.K. received support from the World Health Organization for meta-analysis of this paper. C.D.M. served as a consultant for Otsuka Pharmaceuticals; and received research support from Janssen and
Unitaid. H.S.S. received research support from the U.S. NIH, IMPAACT, and Otsuka Pharmaceuticals. J.R.S. served on a data and safety monitoring board for Otsuka Pharmaceuticals. P.N., G.B.M., T.C., S.R.M., B.S., J.L.B., A.C., J.P.C., L.C., T.L.D., R.D., F.F., F.K., Z.L., A.L., M.L., J.M.M., S.M.M., L.M., D.M., D.M.N., F.P., C.A.P., A.R., N.S.S., G.S., J.W.W., and J.M.W reported no relevant commercial relationships.

Acknowledgment: The authors thank Lindsay McKenna, as part of the writing committee, and the Treatment Action Group, New York, NY, for providing patient advocacy and community engagement input. They also thank Dennis Falzon (World Health Organization) for contributions as an observer. They also thank Kevin Wilson, Kimberly Lawrence, and Judy Corn (American Thoracic Society) Marc Miravitlles and Valérie Vaccaro (European Respiratory Society); Phil LoBue, Angela Starks, Tracy Ayers, Sapna Bamrah Morris (CDC); and Jennifer Padberg (Infectious Diseases Society of America).

\section{References}

1. Barlam TF, Cosgrove SE, Abbo LM, MacDougall C, Schuetz AN, Septimus EJ, et al. Implementing an antibiotic stewardship program: guidelines by the Infectious Diseases Society of America and the Society for Healthcare Epidemiology of America. Clin Infect Dis 2016; 62:e51-e77.

2. U.S. Food and Drug Administration. FDA approves new drug for treatment-resistant forms of tuberculosis that affects the lungs; 2019 [accessed 2019 Sep 23]. Available from: https://www.fda.gov/news-events/press-announcements/fdaapproves-new-drug-treatment-resistant-forms-tuberculosisaffects-lungs.

3. Ahmad N, Ahuja SD, Akkerman OW, Alffenaar JC, Anderson LF, Baghaei $\mathrm{P}$, et al.; Collaborative Group for the Meta-Analysis of Individual Patient Data in MDR-TB treatment-2017. Treatment correlates of successful outcomes in pulmonary multidrug-resistant tuberculosis: an individual patient data meta-analysis. Lancet 2018; 392:821-834.

4. Guyatt GH, Oxman AD, Vist GE, Kunz R, Falck-Ytter $Y$, Alonso-Coello $P$, et al.; GRADE Working Group. GRADE: an emerging consensus on rating quality of evidence and strength of recommendations. $B M J$ 2008;336:924-926.

5. Schünemann HJ, Jaeschke R, Cook DJ, Bria WF, El-Solh AA, Ernst A, et al.; ATS Documents Development and Implementation Committee. An official ATS statement: grading the quality of evidence and strength of recommendations in ATS guidelines and recommendations. Am J Respir Crit Care Med 2006;174:605-614.

6. Silva DR, Rendon A, Alffenaar JW, Chakaya JM, Sotgiu G, Esposito S, et al. Global TB Network: working together to eliminate tuberculosis. $J$ Bras Pneumol 2018;44:347-349.

7. World Health Organization. WHO consolidated guidelines on drugresistant tuberculosis treatment. Geneva, Switzerland: World Health Organization; 2019.

8. Nunn AJ, Phillips PPJ, Meredith SK, Chiang CY, Conradie F, Dalai D, et al.; STREAM Study Collaborators. A trial of a shorter regimen for rifampin-resistant tuberculosis. N Engl J Med 2019;380:1201-1213.

9. D'Ambrosio L, Dara M, Tadolini M, Centis R, Sotgiu G, van der Werf MJ, et al.; European national programme representatives. Tuberculosis elimination: theory and practice in Europe. Eur Respir J 2014;43: 1410-1420.

10. Lönnroth K, Migliori GB, Abubakar I, D'Ambrosio L, de Vries G, Diel R, et al. Towards tuberculosis elimination: an action framework for lowincidence countries. Eur Respir J 2015;45:928-952.

11. Nahid P, Dorman SE, Alipanah N, Barry PM, Brozek JL, Cattamanchi A, et al. Official American Thoracic Society/Centers for Disease Control and Prevention/Infectious Diseases Society of America Clinical Practice Guidelines: treatment of drug-susceptible tuberculosis. Clin Infect Dis 2016;63:e147-e195.

12. World Health Organization. Global tuberculosis report 2018. Geneva, Switzerland: World Health Organization; 2018.

13. Lewinsohn DM, Leonard MK, LoBue PA, Cohn DL, Daley CL, Desmond E, et al. Official American Thoracic Society/Infectious Diseases Society of America/Centers for Disease Control and Prevention Clinical Practice Guidelines: diagnosis of tuberculosis in adults and children. Clin Infect Dis 2017;64:111-115.
14. Clinical and Laboratory Standards Institute (CLSI). Susceptibility testing of mycobacteria, Nocardia spp., and other aerobic actinomycetes, 3rd ed. Wayne, PA: Clinical and Laboratory Standards Institute (CLSI); 2018.

15. International Council of Nurses and Curry International Tuberculosis Center. Nursing guide for managing side effects to drug-resistant TB treatment. 2018 [accessed 2019 Jul 15]. Available from: http:// www.tbonline.info/media/uploads/documents/citc_nursingguide_ english_v7_web_spreads.pdf.

16. Curry International Tuberculosis Center and California Department of Public Health. Drug-resistant tuberculosis: a survival guide for clinicians, 3rd ed. Oakland, CA: Curry International Tuberculosis Center and California Department of Public Health; 2016.

17. Sentinel Project on Pediatric Drug-Resistant Tuberculosis. Management of multidrug-resistant tuberculosis in children: a field guide, 4th ed. Boston, MA: Sentinel Project on Pediatric DrugResistant Tuberculosis; 2019.

18. World Health Organization. Companion handbook to the WHO guidelines for the programmatic management of drug-resistant tuberculosis. Geneva, Switzerland: World Health Organization; 2014.

19. Mitnick CD, White RA, Lu C, Rodriguez CA, Bayona J, Becerra MC, et al.; Collaborative Group for Analysis of Bacteriology Data in MDRTB Treatment. Multidrug-resistant tuberculosis treatment failure detection depends on monitoring interval and microbiological method. Eur Respir J 2016;48:1160-1170.

20. American Thoracic Society; Centers for Disease Control and Prevention; Infectious Diseases Society of America. American Thoracic Society/Centers for Disease Control and Prevention/ Infectious Diseases Society of America: controlling tuberculosis in the United States. Am J Respir Crit Care Med 2005;172:1169-1227.

21. World Health Organization. Who policy on TB infection control in health-care facilities, congregate settings and households: Geneva, Switzerland: World Health Organization; 2009.

22. Bothamley GH, Lange C; TBNET. Infection control, genetic assessment of drug resistance and drug susceptibility testing in the current management of multidrug/extensively-resistant tuberculosis (M/XDR-TB) in Europe: a tuberculosis network European Trialsgroup (TBNET) study. Respir Med 2017;132:68-75.

23. World Health Organization. Who treatment guidelines for drug-resistant tuberculosis 2016 update. Geneva, Switzerland: World Health Organization; 2016

24. Migliori GB, Nardell E, Yedilbayev A, D'Ambrosio L, Centis R, Tadolini $M$, et al. Reducing tuberculosis transmission: a consensus document from the world health organization regional office for Europe. Eur Respir J 2019;53:1900391.

25. Melsew YA, Doan TN, Gambhir M, Cheng AC, McBryde E, Trauer JM. Risk factors for infectiousness of patients with tuberculosis: a systematic review and meta-analysis. Epidemiol Infect 2018;146:345-353.

26. Dharmadhikari AS, Mphahlele M, Venter K, Stoltz A, Mathebula R, Masotla T, et al. Rapid impact of effective treatment on transmission of multidrug-resistant tuberculosis. Int J Tuberc Lung Dis 2014;18: 1019-1025.

27. Jensen PA, Lambert LA, lademarco MF, Ridzon R; CDC. Guidelines for preventing the transmission of Mycobacterium tuberculosis in health-care settings, 2005. MMWR Recomm Rep 2005;54:1-141. 
28. Case Management Society of America. Standards of practice for case management; 2016 [accessed 2019 Sep 15]. Available from: http://www.naylornetwork.com/cmsatoday/articles/index-v3.asp? aid $=400028$ \&issuelD $=53653$.

29. Hickam DH, Weiss JW, Guise JM, Buckley D, Motu'apuaka M, Graham $\mathrm{E}$, et al. Outpatient case management for adults with medical illness and complex care needs. Rockville, MD: CreateSpace Independent Publishing Platform; 2013.

30. Ross S, Goodwin N, Curry N. Case management: what is it and how it can best be implemented. London, UK: King's Fund; 2011.

31. Klopf LC. Tuberculosis control in the New York State Department of Correctional Services: a case management approach. Am J Infect Control 1998;26:534-537.

32. Advisory Council for the Elimination of Tuberculosis. Essential components of a tuberculosis prevention and control program: recommendations of the Advisory Council for the Elimination of Tuberculosis. MMWR Recomm Rep 1995;44:1-16.

33. Epstein RM, Street RL Jr. The values and value of patient-centered care. Ann Fam Med 2011;9:100-103.

34. Migliori GB, Sotgiu G, Rosales-Klintz S, Centis R, D'Ambrosio L, Abubakar I, et al. ERS/ECDC Statement: European Union standards for tuberculosis care, 2017 update. Eur Respir J 2018; $51: 1702678$.

35. TB CARE I. International standards for tuberculosis care, 3rd ed. The Hague, the Netherlands: TB CARE I.; 2018 [accessed 2014 Jul 15]. Available from: http://www.who.int/tb/publications/ISTC_3rdEd.pdf.

36. Robinson JH, Callister LC, Berry JA, Dearing KA. Patient-centered care and adherence: definitions and applications to improve outcomes. $J$ Am Acad Nurse Pract 2008;20:600-607.

37. National Tuberculosis Controllers Association. Tuberculosis nursing: a comprehensive guide to patient care, 2 nd ed. Smyrna, GA: National Tuberculosis Controllers Association; 2011.

38. Seaworth BJ. Multidrug-resistant tuberculosis. Infect Dis Clin North Am 2002;16:73-105.

39. Alipanah N, Jarlsberg L, Miller C, Linh NN, Falzon D, Jaramillo E, et al. Adherence interventions and outcomes of tuberculosis treatment: a systematic review and meta-analysis of trials and observational studies. PLoS Med 2018;15:e1002595.

40. Müller AM, Osório CS, Silva DR, Sbruzzi G, de Tarso P, Dalcin R. Interventions to improve adherence to tuberculosis treatment: systematic review and meta-analysis. Int J Tuberc Lung Dis 2018;22: 731-740.

41. Falzon D, Timimi $H$, Kurosinski $P$, Migliori GB, Van Gemert W, Denkinger C, et al. Digital health for the End TB Strategy: developing priority products and making them work. Eur Respir J 2016;48: $29-45$.

42. Sinkou H, Hurevich H, Rusovich V, Zhylevich L, Falzon D, de Colombani $\mathrm{P}$, et al. Video-observed treatment for tuberculosis patients in Belarus: findings from the first programmatic experience. Eur Respir J 2017;49:1602049.

43. Chuck C, Robinson E, Macaraig M, Alexander M, Burzynski J. Enhancing management of tuberculosis treatment with video directly observed therapy in New York City. Int J Tuberc Lung Dis 2016;20: 588-593.

44. Story A, Garfein RS, Hayward A, Rusovich V, Dadu A, Soltan V, et al. Monitoring therapy compliance of tuberculosis patients by using video-enabled electronic devices. Emerg Infect Dis 2016;22: 538-540.

45. Zúñiga ML, Collins K, Muñoz F, Moser K, Rangel G, Cuevas-Mota J, et al. A qualitative study exploring stakeholder perceptions of video directly observed therapy for monitoring tuberculosis treatment in the US-Mexico border region. J Mob Technol Med 2016; 5:12-23.

46. Garfein RS, Collins K, Muñoz F, Moser K, Cerecer-Callu P, Raab F, et al. Feasibility of tuberculosis treatment monitoring by video directly observed therapy: a binational pilot study. Int $J$ Tuberc Lung Dis 2015;19:1057-1064.

47. World Health Organization. Digital health for the End TB Strategy: an agenda for action. Geneva, Switzerland: World Health Organization; 2015.
48. Velayutham B, Nair D, Ramalingam S, Perez-Velez CM, Becerra MC, Swaminathan S. Setting priorities for a research agenda to combat drug-resistant tuberculosis in children. Public Health Action 2015;5: 222-235.

49. Garfein RS, Liu L, Cuevas-Mota J, Collins K, Muñoz F, Catanzaro DG et al. Tuberculosis treatment monitoring by video directly observed therapy in 5 health districts, California, USA. Emerg Infect Dis 2018; 24:1806-1815.

50. Schnippel K, Ndjeka N, Maartens G, Meintjes G, Master I, Ismail N, et al. Effect of bedaquiline on mortality in South African patients with drug-resistant tuberculosis: a retrospective cohort study. Lancet Respir Med 2018;6:699-706.

51. Seddon JA, Schaaf HS, Marais BJ, McKenna L, Garcia-Prats AJ, Hesseling AC, et al. Time to act on injectable-free regimens for children with multidrug-resistant tuberculosis. Lancet Respir Med 2018;6:662-664.

52. Günther G, Lange C, Alexandru S, Altet N, Avsar K, Bang D, et al.; for TBNET. Treatment outcomes in multidrug-resistant tuberculosis. $N$ Engl J Med 2016;375:1103-1105.

53. Goble M, Iseman MD, Madsen LA, Waite D, Ackerson L, Horsburgh CR Jr. Treatment of 171 patients with pulmonary tuberculosis resistant to isoniazid and rifampin. N Engl J Med 1993;328: 527-532.

54. Falzon $\mathrm{D}$, Jaramillo $\mathrm{E}$, Schünemann $\mathrm{HJ}$, Arentz $\mathrm{M}$, Bauer M, Bayona J, et al. WHO guidelines for the programmatic management of drug-resistant tuberculosis: 2011 update. Eur Respir J 2011;38: 516-528.

55. World Health Organization. Guidelines for the programmatic management of drug-resistant tuberculosis. Geneva, Switzerland: World Health Organization; 2008.

56. Tiberi S, du Plessis N, Walzl G, Vjecha MJ, Rao M, Ntoumi F, et al. Tuberculosis: progress and advances in development of new drugs, treatment regimens, and host-directed therapies. Lancet Infect Dis 2018;18:e183-e198.

57. Imperial MZ, Nahid P, Phillips PPJ, Davies GR, Fielding K, Hanna D, et al. A patient-level pooled analysis of treatment-shortening regimens for drug-susceptible pulmonary tuberculosis. Nat Med 2018;24:1708-1715.

58. Kasik JE. The nature of mycobacterial penicillinase. Am Rev Respir Dis 1965;91:117-119.

59. Seung KJ, Becerra MC, Atwood SS, Alcántara F, Bonilla CA, Mitnick CD. Salvage therapy for multidrug-resistant tuberculosis. Clin Microbiol Infect 2014;20:441-446.

60. Donald PR, Sirgel FA, Venter A, Parkin DP, Van de Wal BW, Barendse $A$, et al. Early bactericidal activity of amoxicillin in combination with clavulanic acid in patients with sputum smearpositive pulmonary tuberculosis. Scand J Infect Dis 2001;33: 466-469.

61. Chambers HF, Kocagöz T, Sipit T, Turner J, Hopewell PC. Activity of amoxicillin/clavulanate in patients with tuberculosis. Clin Infect Dis 1998;26:874-877.

62. Nadler JP, Berger J, Nord JA, Cofsky R, Saxena M. Amoxicillinclavulanic acid for treating drug-resistant Mycobacterium tuberculosis. Chest 1991;99:1025-1026.

63. Gonzalo X, Drobniewski F. Is there a place for $\beta$-lactams in the treatment of multidrug-resistant/extensively drugresistant tuberculosis? Synergy between meropenem and amoxicillin/clavulanate. J Antimicrob Chemother 2013;68: 366-369.

64. Dauby N, Muylle I, Mouchet F, Sergysels R, Payen MC. Meropenem/clavulanate and linezolid treatment for extensively drug-resistant tuberculosis. Pediatr Infect Dis J 2011;30: 812-813.

65. Sotgiu G, D’Ambrosio L, Centis R, Tiberi S, Esposito S, Dore S, et al. Carbapenems to treat multidrug and extensively drugresistant tuberculosis: a systematic review. Int J Mol Sci 2016; 17:373.

66. Tiberi S, Payen MC, Sotgiu G, D'Ambrosio L, Alarcon Guizado V, Alffenaar JW, et al. Effectiveness and safety of meropenem/clavulanate-containing regimens in the treatment of MDR- and XDR-TB. Eur Respir J 2016;47:1235-1243. 
67. De Lorenzo S, Alffenaar JW, Sotgiu G, Centis R, D'Ambrosio L, Tiberi S, et al. Efficacy and safety of meropenem-clavulanate added to linezolid-containing regimens in the treatment of MDR-/XDR-TB. Eur Respir J 2013;41:1386-1392.

68. Swaminathan A, du Cros P, Seddon JA, Quinnell S, Bobokhojaev OI, Dusmatova Z, et al. Treating children for drug-resistant tuberculosis in Tajikistan with Group 5 medications. Int J Tuberc Lung Dis 2016; 20:474-478.

69. Ahmed I, Jabeen K, Inayat R, Hasan R. Susceptibility testing of extensively drug-resistant and pre-extensively drug-resistant Mycobacterium tuberculosis against levofloxacin, linezolid, and amoxicillin-clavulanate. Antimicrob Agents Chemother 2013;57: 2522-2525.

70. Gillies M, Ranakusuma A, Hoffmann T, Thorning S, McGuire T, Glasziou $\mathrm{P}$, et al. Common harms from amoxicillin: a systematic review and meta-analysis of randomized placebo-controlled trials for any indication. CMAJ 2015;187:E21-E31.

71. Mase S, Chorba T, Lobue P, Castro K; Centers for Disease Control and Prevention. Provisional CDC guidelines for the use and safety monitoring of bedaquiline fumarate (Sirturo) for the treatment of multidrug-resistant tuberculosis. MMWR Recomm Rep 2013;62:1-12.

72. U.S. Food and Drug Administration. Package insert: Sirturo [accessed 2019 Oct 26]. Available from: https://www.accessdata.fda.gov/ drugsatfda_docs/label/2019/204384s010lbl.pdf.

73. Andries K, Verhasselt P, Guillemont J, Göhlmann HW, Neefs JM, Winkler $\mathrm{H}$, et al. A diarylquinoline drug active on the ATP synthase of Mycobacterium tuberculosis. Science 2005;307:223-227.

74. Almeida D, loerger T, Tyagi S, Li SY, Mdluli K, Andries K, et al. Mutations in pepQ confer low-level resistance to bedaquiline and clofazimine in mycobacterium tuberculosis. Antimicrob Agents Chemother 2016;60:4590-4599.

75. Nguyen TVA, Anthony RM, Bañuls AL, Nguyen TVA, Vu DH, Alffenaar JC. Bedaquiline resistance: its emergence, mechanism, and prevention. Clin Infect Dis 2018;66:1625-1630.

76. Bedaquiline (Sirturo) for multidrug-resistant tuberculosis. Med Lett Drugs Ther 2013;55:66-68.

77. Pontali E, D’Ambrosio L, Centis R, Sotgiu G, Migliori GB. Multidrug-resistant tuberculosis and beyond: an updated analysis of the current evidence on bedaquiline. Eur Respir $J$ 2017;49:1700146.

78. Pontali E, Sotgiu G, D’Ambrosio L, Centis R, Migliori GB. Bedaquiline and multidrug-resistant tuberculosis: a systematic and critical analysis of the evidence. Eur Respir J 2016;47:394-402.

79. World Health Organization. Rapid communication: key changes to treatment of multidrug- and rifampicin-resistant tuberculosis (MDR/RR-TB). Geneva, Switzerland: World Health Organization; 2018.

80. Borisov SE, Dheda K, Enwerem M, Romero Leyet R, D'Ambrosio L, Centis R, et al. Effectiveness and safety of bedaquiline-containing regimens in the treatment of MDR- and XDR-TB: a multicentre study. Eur Respir J 2017;49:1700387.

81. Pontali E, Sotgiu G, Tiberi S, D'Ambrosio L, Centis R, Migliori GB. Cardiac safety of bedaquiline: a systematic and critical analysis of the evidence. Eur Respir J 2017;50:1701462.

82. Achar J, Hewison C, Cavalheiro AP, Skrahina A, Cajazeiro J, Nargiza P, et al. Off-label use of bedaquiline in children and adolescents with multidrug-resistant tuberculosis. Emerg Infect Dis 2017;23: 10.3201/eid2310.170303.

83. Harausz EP, Garcia-Prats AJ, Seddon JA, Schaaf HS, Hesseling AC, Achar J, et al.; Sentinel Project on Pediatric Drug-Resistant Tuberculosis. New and repurposed drugs for pediatric multidrugresistant tuberculosis: practice-based recommendations. Am J Respir Crit Care Med 2017;195:1300-1310.

84. Lewis JM, Hine P, Walker J, Khoo SH, Taegtmeyer M, Squire SB, et al. First experience of effectiveness and safety of bedaquiline for 18 months within an optimised regimen for XDR-TB. Eur Respir J 2016; 47:1581-1584.

85. England K, Boshoff HI, Arora K, Weiner D, Dayao E, Schimel D, et al. Meropenem-clavulanic acid shows activity against Mycobacterium tuberculosis in vivo. Antimicrob Agents Chemother 2012;56: 3384-3387.
86. Hugonnet JE, Tremblay LW, Boshoff HI, Barry CE III, Blanchard JS. Meropenem-clavulanate is effective against extensively drugresistant Mycobacterium tuberculosis. Science 2009;323: $1215-1218$.

87. Fox GJ, Benedetti A, Cox H, Koh WJ, Viiklepp P, Ahuja S, et al.; Collaborative Group for Meta-Analysis of Individual Patient Data in MDR-TB. Group 5 drugs for multidrug-resistant tuberculosis: individual patient data meta-analysis. Eur Respir J 2017;49: 1600993.

88. Mafukidze A, Harausz E, Furin J. An update on repurposed medications for the treatment of drug-resistant tuberculosis. Expert Rev Clin Pharmacol 2016;9:1331-1340.

89. Tiberi S, Sotgiu G, D'Ambrosio L, Centis R, Arbex MA, Alarcon Arrascue $\mathrm{E}$, et al. Effectiveness and safety of imipenem-clavulanate added to an optimized background regimen (OBR) versus OBR control regimens in the treatment of multidrug-resistant and extensively drug-resistant tuberculosis. Clin Infect Dis 2016;62: 1188-1190.

90. Tiberi S, Sotgiu G, D’Ambrosio L, Centis R, Abdo Arbex M, Alarcon Arrascue $\mathrm{E}$, et al. Comparison of effectiveness and safety of imipenem/clavulanate- versus meropenem/clavulanate-containing regimens in the treatment of MDR- and XDR-TB. Eur Respir J 2016; 47:1758-1766.

91. van Rijn SP, van Altena R, Akkerman OW, van Soolingen D, van der Laan $\mathrm{T}$, de Lange WC, et al. Pharmacokinetics of ertapenem in patients with multidrug-resistant tuberculosis. Eur Respir J 2016;47:1229-1234.

92. Tiberi S, D’Ambrosio L, De Lorenzo S, Viggiani P, Centis R, Sotgiu G, et al. Ertapenem in the treatment of multidrug-resistant tuberculosis: first clinical experience. Eur Respir J 2016;47:333-336.

93. Sotgiu G, Tiberi S, D'Ambrosio L, Centis R, Alffenaar JW, Caminero JA, et al.; International Carbapenem Study Group. Faster for less: the new "shorter" regimen for multidrug-resistant tuberculosis. Eur Respir J 2016;48:1503-1507.

94. Tang S, Yao L, Hao X, Liu Y, Zeng L, Liu G, et al. Clofazimine for the treatment of multidrug-resistant tuberculosis: prospective, multicenter, randomized controlled study in China. Clin Infect Dis 2015;60:1361-1367.

95. Gopal M, Padayatchi N, Metcalfe JZ, O'Donnell MR. Systematic review of clofazimine for the treatment of drug-resistant tuberculosis. Int $J$ Tuberc Lung Dis 2013;17:1001-1007.

96. Falzon D, Schünemann HJ, Harausz E, González-Angulo L, Lienhardt $\mathrm{C}$, Jaramillo $\mathrm{E}$, et al. World Health Organization treatment guidelines for drug-resistant tuberculosis, 2016 update. Eur Respir J 2017;49: 1602308

97. Grosset JH, Tyagi S, Almeida DV, Converse PJ, Li SY, Ammerman NC, et al. Assessment of clofazimine activity in a second-line regimen for tuberculosis in mice. Am J Respir Crit Care Med 2013;188: 608-612.

98. Lechartier B, Cole ST. Mode of action of clofazimine and combination therapy with benzothiazinones against mycobacterium tuberculosis. Antimicrob Agents Chemother 2015;59:4457-4463.

99. Hwang TJ, Dotsenko S, Jafarov A, Weyer K, Falzon D, Lunte K, et al. Safety and availability of clofazimine in the treatment of multidrug and extensively drug-resistant tuberculosis: analysis of published guidance and meta-analysis of cohort studies. BMJ Open 2014;4: e004143.

100. World Health Organization. Position statement on the continued use of the shorter MDR-TB regimen following an expedited review of the STREAM Stage 1 preliminary results. Geneva, Switzerland: World Health Organization; 2018.

101. Diel R, Vandeputte J, de Vries G, Stillo J, Wanlin M, Nienhaus A. Costs of tuberculosis disease in the European Union: a systematic analysis and cost calculation. Eur Respir J 2014;43: 554-565.

102. Aung KJ, Van Deun A, Declercq E, Sarker MR, Das PK, Hossain MA, et al. Successful '9-month Bangladesh regimen' for multidrugresistant tuberculosis among over 500 consecutive patients. Int $J$ Tuberc Lung Dis 2014;18:1180-1187.

103. Van Deun A, Maug AK, Salim MA, Das PK, Sarker MR, Daru P, et al. Short, highly effective, and inexpensive standardized treatment of multidrug-resistant tuberculosis. Am J Respir Crit Care Med 2010; 182:684-692. 
104. Piubello A, Harouna SH, Souleymane MB, Boukary I, Morou S, Daouda $\mathrm{M}$, et al. High cure rate with standardised short-course multidrug-resistant tuberculosis treatment in Niger: no relapses. Int $J$ Tuberc Lung Dis 2014;18:1188-1194.

105. Kuaban C, Noeske J, Rieder HL, Aït-Khaled N, Abena Foe JL, Trébucq A. High effectiveness of a 12-month regimen for MDR-TB patients in Cameroon. Int J Tuberc Lung Dis 2015;19: 517-524.

106. Javaid A, Ahmad N, Khan AH, Shaheen Z. Applicability of the World Health Organization recommended new shorter regimen in a multidrug-resistant tuberculosis high burden country. Eur Respir $J$ 2017;49:1601967.

107. van der Werf MJ, Hollo V, Ködmön C, Dara M, Catchpole M. Eligibility for shorter treatment of multidrug-resistant tuberculosis in the European Union. Eur Respir J 2017;49:1601992.

108. Dalcolmo M, Gayoso R, Sotgiu G, D'Ambrosio L, Rocha JL, Borga L, et al. Resistance profile of drugs composing the "shorter" regimen for multidrug-resistant tuberculosis in Brazil, 2000-2015. Eur Respir J 2017;49:1602309.

109. Dalcolmo M, Gayoso R, Sotgiu G, D'Ambrosio L, Rocha JL, Borga L, et al. Effectiveness and safety of clofazimine in multidrug-resistant tuberculosis: a nationwide report from Brazil. Eur Respir J 2017;49: 1602445.

110. Tadolini M, Lingtsang RD, Tiberi S, Enwerem M, D'Ambrosio L, Sadutshang TD, et al. Cardiac safety of extensively drug-resistant tuberculosis regimens including bedaquiline, delamanid and clofazimine. Eur Respir J 2016;48:1527-1529.

111. Wallis RS. Cardiac safety of extensively drug-resistant tuberculosis regimens including bedaquiline, delamanid and clofazimine. Eur Respir J 2016;48:1526-1527.

112. Diacon AH, Dawson $R$, von Groote-Bidlingmaier F, Symons G, Venter A, Donald PR, et al. Bactericidal activity of pyrazinamide and clofazimine alone and in combinations with pretomanid and bedaquiline. Am J Respir Crit Care Med 2015; 191:943-953.

113. Harausz EP, Garcia-Prats AJ, Law S, Schaaf HS, Kredo T, Seddon JA, et al.; Collaborative Group for Meta-Analysis of Paediatric Individual Patient Data in MDR-TB. Treatment and outcomes in children with multidrug-resistant tuberculosis: a systematic review and individual patient data meta-analysis. PLoS Med 2018;15: e1002591.

114. Kroger A, Pannikar V, Htoon MT, Jamesh A, Katoch K, Krishnamurthy $\mathrm{P}$, et al. International open trial of uniform multi-drug therapy regimen for 6 months for all types of leprosy patients: rationale, design and preliminary results. Trop Med Int Health 2008;13: 594-602.

115. Schaaf HS, Garcia-Prats AJ, McKenna L, Seddon JA. Challenges of using new and repurposed drugs for the treatment of multidrugresistant tuberculosis in children. Expert Rev Clin Pharmacol 2018; 11:233-244.

116. Bruning JB, Murillo AC, Chacon O, Barletta RG, Sacchettini JC. Structure of the Mycobacterium tuberculosis D-alanine:D-alanine ligase, a target of the antituberculosis drug D-cycloserine. Antimicrob Agents Chemother 2011;55:291-301.

117. Cycloserine. Tuberculosis (Edinb) 2008;88:100-101

118. Hwang TJ, Wares DF, Jafarov A, Jakubowiak W, Nunn P, Keshavjee S. Safety of cycloserine and terizidone for the treatment of drugresistant tuberculosis: a meta-analysis. Int J Tuberc Lung Dis 2013; 17:1257-1266.

119. Hemanth Kumar AK, Kumar A, Kannan T, Bhatia R, Agarwal D, Kumar $\mathrm{S}$, et al. Pharmacokinetics of second-line antituberculosis drugs in children with multidrug-resistant tuberculosis in India. Antimicrob Agents Chemother 2018;62:e02410-17.

120. Ramachandran G, Swaminathan S. Safety and tolerability profile of second-line anti-tuberculosis medications. Drug Saf 2015;38: 253-269.

121. Schloss J, Ismail Z. Cycloserine and isoniazid in childhood tuberculous infections. Antibiotic Med Clin Ther (New York) 1960;7: 244-248.

122. Steiner M. Newer and second-line drugs in the treatment of drugresistant tuberculosis in children. Med Clin North Am 1967;51: 1153-1167.
123. Battaglia B, Kaufman I, Lyons HA, Marsh W. Toxicity of cycloserine combined with isoniazid in the treatment of tuberculosis in children. Am Rev Respir Dis 1961;83:751-752.

124. Ettehad D, Schaaf HS, Seddon JA, Cooke GS, Ford N. Treatment outcomes for children with multidrug-resistant tuberculosis: a systematic review and meta-analysis. Lancet Infect Dis 2012;12: 449-456.

125. Hung WY, Yu MC, Chiang YC, Chang JH, Chiang CY, Chang CC, et al. Serum concentrations of cycloserine and outcome of multidrug-resistant tuberculosis in Northern Taiwan. Int $J$ Tuberc Lung Dis 2014;18:601-606.

126. World Health Organization. The use of delamanid in the treatment of multidrug-resistant tuberculosis: interim policy guidance. Geneva, Switzerland: World Health Organization; 2014.

127. von Groote-Bidlingmaier F, Patientia R, Sanchez E, Balanag V Jr, Ticona E, Segura P, et al. Efficacy and safety of delamanid in combination with an optimised background regimen for treatment of multidrug-resistant tuberculosis: a multicentre, randomised, double-blind, placebo-controlled, parallel group phase 3 trial. Lancet Respir Med 2019;7:249-259.

128. World Health Organization. WHO position statement on the use of delamanid for multidrug-resistant tuberculosis. Geneva, Switzerland: World Health Organization; 2018.

129. Mohr E, Hughes J, Reuter A, Trivino Duran L, Ferlazzo G, Daniels J, et al. Delamanid for rifampicin-resistant tuberculosis: a retrospective study from South Africa. Eur Respir J 2018;51: 1800017.

130. Kuksa L, Barkane L, Hittel N, Gupta R. Final treatment outcomes of multidrug- and extensively drug-resistant tuberculosis patients in Latvia receiving delamanid-containing regimens. Eur Respir J 2017; 50:1701105.

131. Centis R, Sotgiu G, Migliori GB. Delamanid: does it have a role in tuberculosis treatment? Lancet Respir Med 2019;7: 193-195.

132. Lardizabal AA, Khan AN, Bamrah Morris S, Goswami ND. Notes from the field: acquisition of delamanid under a compassionate use program for extensively drug-resistant tuberculosis United States, 2017. MMWR Morb Mortal Wkly Rep 2018;67: 996-997.

133. Tadolini M, Garcia-Prats AJ, D'Ambrosio L, Hewison C, Centis R, Schaaf HS, et al. Compassionate use of new drugs in children and adolescents with multidrug-resistant and extensively drug-resistant tuberculosis: early experiences and challenges. Eur Respir J 2016; 48:938-943.

134. D’Ambrosio L, Centis R, Tiberi S, Tadolini M, Dalcolmo M, Rendon A, et al. Delamanid and bedaquiline to treat multidrug-resistant and extensively drug-resistant tuberculosis in children: a systematic review. J Thorac Dis 2017;9:2093-2101.

135. World Health Organization. The use of delamanid in the treatment of multidrug-resistant tuberculosis in children and adolescents: interim policy guidance. Geneva, Switzerland: World Health Organization; 2016.

136. Dooley K, Rosenkranz S, Conradie F, Moran L, Hafner R, von Groote-Bidlingmaier F, et al. QT effects of bedaquiline, delamanid or both in MDR-TB patients: the deliberate trial. Presented at the Conference on Retroviruses and Opportunistic Infections (CROI). March 4-7, 2019, Seattle, WA. Abstract 84

137. Tadolini M, Lingtsang RD, Tiberi S, Enwerem M, D'Ambrosio L, Sadutshang TD, et al. First case of extensively drug-resistant tuberculosis treated with both delamanid and bedaquiline. Eur Respir J 2016;48:935-938.

138. Ferlazzo G, Mohr E, Laxmeshwar C, Hewison C, Hughes J, Jonckheere $\mathrm{S}$, et al. Early safety and efficacy of the combination of bedaquiline and delamanid for the treatment of patients with drug-resistant tuberculosis in Armenia, India, and South Africa: a retrospective cohort study. Lancet Infect Dis 2018; 18:536-544.

139. Kim CT, Kim TO, Shin HJ, Ko YC, Hun Choe Y, Kim HR, et al. Bedaquiline and delamanid for the treatment of multidrug-resistant tuberculosis: a multicentre cohort study in Korea. Eur Respir $J$ 2018;51:1702467. 
140. Pontali E, Sotgiu G, Tiberi S, Tadolini M, Visca D, D’Ambrosio L, et al. Combined treatment of drug-resistant tuberculosis with bedaquiline and delamanid: a systematic review. Eur Respir J 2018; 52:1800934.

141. U.S. Food and Drug Administration. Pretomanid tablet, $200 \mathrm{mg}$ meeting of the Antimicrobial Drugs Advisory Committee (AMDAC): June 6, 2019; 2019 [accessed 2019 Jul 9]. Available from: https://www.fda.gov/advisory-committees/advisory-committeecalendar/june-6-2019-antimicrobial-drugs-advisory-committeemeeting-announcement-06062019-06062019.

142. Goude R, Amin AG, Chatterjee D, Parish T. The arabinosyltransferase EmbC is inhibited by ethambutol in Mycobacterium tuberculosis. Antimicrob Agents Chemother 2009;53:4138-4146.

143. Donald P, Maher D, Maritz S, Qazi SA; World Health Organization. Ethambutol efficacy and toxicity: literature review and recommendations for daily and intermittent dosage in children. Geneva, Switzerland: World Health Organization; 2006.

144. Bastos ML, Hussain H, Weyer K, Garcia-Garcia L, Leimane V, Leung $\mathrm{CC}$, et al.; Collaborative Group for Meta-analysis of Individual Patient Data in MDR-TB. Treatment outcomes of patients with multidrug-resistant and extensively drug-resistant tuberculosis according to drug susceptibility testing to first- and second-line drugs: an individual patient data meta-analysis. Clin Infect Dis 2014; 59:1364-1374.

145. Cegielski JP, Dalton T, Yagui M, Wattanaamornkiet W, Volchenkov GV, Via LE, et al.; Global Preserving Effective TB Treatment Study (PETTS) Investigators. Extensive drug resistance acquired during treatment of multidrug-resistant tuberculosis. Clin Infect Dis 2014; 59:1049-1063.

146. Graham SM, Daley HM, Banerjee A, Salaniponi FM, Harries AD. Ethambutol in tuberculosis: time to reconsider? Arch Dis Child 1998;79:274-278.

147. Scardigli A, Caminero JA, Sotgiu G, Centis R, D'Ambrosio L, Migliori GB. Efficacy and tolerability of ethionamide versus prothionamide: a systematic review. Eur Respir J 2016;48: 946-952.

148. Thee S, Garcia-Prats AJ, Donald PR, Hesseling AC, Schaaf HS. A review of the use of ethionamide and prothionamide in childhood tuberculosis. Tuberculosis (Edinb) 2016;97:126-136.

149. Ahuja SD, Ashkin D, Avendano M, Banerjee R, Bauer M, Bayona JN, et al.; Collaborative Group for Meta-Analysis of Individual Patient Data in MDR-TB. Multidrug resistant pulmonary tuberculosis treatment regimens and patient outcomes: an individual patient data meta-analysis of 9,153 patients. PLoS Med 2012;9:e1001300.

150. Dutta BS, Hassan G, Waseem Q, Saheer S, Singh A. Ethionamideinduced hypothyroidism. Int J Tuberc Lung Dis 2012;16:141.

151. McDonnell ME, Braverman LE, Bernardo J. Hypothyroidism due to ethionamide. N Engl J Med 2005;352:2757-2759.

152. Thee S, Zöllner EW, Willemse M, Hesseling AC, Magdorf K, Schaaf HS. Abnormal thyroid function tests in children on ethionamide treatment. Int J Tuberc Lung Dis 2011;15:1191-1193, i.

153. Morlock GP, Metchock B, Sikes D, Crawford JT, Cooksey RC. ethA, inhA, and katG loci of ethionamide-resistant clinical Mycobacterium tuberculosis isolates. Antimicrob Agents Chemother 2003;47: 3799-3805.

154. Ling LL, Schneider T, Peoples AJ, Spoering AL, Engels I, Conlon BP, et al. A new antibiotic kills pathogens without detectable resistance. Nature 2015;517:455-459.

155. Bennett JE, Dolin R, Blaser MJ. Mandell, Douglas, and Bennett's principles and practice of infectious diseases e-book. Philadelphia, PA: Elsevier Health Sciences; 2014.

156. Angeby KA, Jureen $P$, Giske CG, Chryssanthou $E$, Sturegård $E$, Nordvall $M$, et al. Wild-type MIC distributions of four fluoroquinolones active against Mycobacterium tuberculosis in relation to current critical concentrations and available pharmacokinetic and pharmacodynamic data. J Antimicrob Chemother 2010;65:946-952.

157. Peloquin CA, Hadad DJ, Molino LP, Palaci M, Boom WH, Dietze R, et al. Population pharmacokinetics of levofloxacin, gatifloxacin, and moxifloxacin in adults with pulmonary tuberculosis. Antimicrob Agents Chemother 2008;52:852-857.
158. Chan ED, Laurel V, Strand MJ, Chan JF, Huynh ML, Goble M, et al. Treatment and outcome analysis of 205 patients with multidrugresistant tuberculosis. Am J Respir Crit Care Med 2004;169: 1103-1109.

159. Falzon D, Gandhi N, Migliori GB, Sotgiu G, Cox HS, Holtz TH, et al.; Collaborative Group for Meta-Analysis of Individual Patient Data in MDR-TB. Resistance to fluoroquinolones and second-line injectable drugs: impact on multidrug-resistant TB outcomes. Eur Respir J 2013;42:156-168.

160. Cegielski JP, Kurbatova E, van der Walt M, Brand J, Ershova J, Tupasi T, et al.; Global PETTS Investigators. Multidrug-resistant tuberculosis treatment outcomes in relation to treatment and initia versus acquired second-line drug resistance. Clin Infect Dis 2016; 62:418-430.

161. Seddon JA, Hesseling AC, Godfrey-Faussett P, Schaaf HS. High treatment success in children treated for multidrug-resistant tuberculosis: an observational cohort study. Thorax 2014;69: 458-464.

162. American Society of Hospital Pharmacists (AHFS). AHFS drug information. Bethesda, MD: Board of Directors of the American Society of Hospital Pharmacists; 2018.

163. U.S. Food and Drug Administration. FDA safety announcement: FDA warns about increased risk of ruptures or tears in the aorta blood vessel with fluoroquinolone antibiotics in certain patients; 2018 [accessed 2019 Apr 7]. Available from: https://www.fda.gov/ Drugs/DrugSafety/ucm628753.htm.

164. Bradley JS, Kauffman RE, Balis DA, Duffy CM, Gerbino PG, Maldonado $\mathrm{SD}$, et al. Assessment of musculoskeletal toxicity 5 years after therapy with levofloxacin. Pediatrics 2014;134:e146-e153.

165. Garcia-Prats AJ, Draper HR, Finlayson H, Winckler J, Burger A, Fourie $B$, et al. Clinical and cardiac safety of long-term levofloxacin in children treated for multidrug-resistant tuberculosis. Clin Infect Dis 2018;67:1777-1780.

166. Seddon JA, Hesseling AC, Finlayson H, Fielding K, Cox H, Hughes J, et al. Preventive therapy for child contacts of multidrug-resistant tuberculosis: a prospective cohort study. Clin Infect Dis 2013;57: 1676-1684.

167. Stass H, Kubitza D. Effects of dairy products on the oral bioavailability of moxifloxacin, a novel 8-methoxyfluoroquinolone, in healthy volunteers. Clin Pharmacokinet 2001;40:33-38.

168. Sörgel F, Kinzig M. Pharmacokinetics of gyrase inhibitors, Part 1: basic chemistry and gastrointestinal disposition. Am J Med 1993; 94:44S-55S

169. Staib AH, Beermann D, Harder S, Fuhr U, Liermann D. Absorption differences of ciprofloxacin along the human gastrointestinal tract determined using a remote-control drug delivery device (HFcapsule). Am J Med 1989;87:66S-69S.

170. Denti P, Garcia-Prats AJ, Draper HR, Wiesner L, Winckler J, Thee S, et al. Levofloxacin population pharmacokinetics in South African children treated for multidrug-resistant tuberculosis. Antimicrob Agents Chemother 2018;62:e01521-17.

171. Mase SR, Jereb JA, Gonzalez D, Martin F, Daley CL, Fred D, et al. Pharmacokinetics and dosing of levofloxacin in children treated for active or latent multidrug-resistant tuberculosis, Federated States of Micronesia and republic of the Marshall Islands. Pediatr Infect Dis J 2016;35:414-421.

172. Thee S, Garcia-Prats AJ, Draper HR, McIlleron HM, Wiesner L, Castel $\mathrm{S}$, et al. Pharmacokinetics and safety of moxifloxacin in children with multidrug-resistant tuberculosis. Clin Infect Dis 2015;60: 549-556.

173. Srivastava S, Deshpande D, Pasipanodya J, Nuermberger E, Swaminathan S, Gumbo T. Optimal clinical doses of faropenem, linezolid, and moxifloxacin in children with disseminated tuberculosis: Goldilocks. Clin Infect Dis 2016; 63:S102-S109.

174. Peloquin CA, Phillips PPJ, Mitnick CD, Eisenach K, Patientia RF, Lecca $L$, et al. Increased doses lead to higher drug exposures of levofloxacin for treatment of tuberculosis. Antimicrob Agents Chemother 2018;62:e00770-18.

175. Drusano GL, Louie A, Deziel M, Gumbo T. The crisis of resistance: identifying drug exposures to suppress amplification of resistant mutant subpopulations. Clin Infect Dis 2006;42:525-532. 
176. Gumbo T, Louie A, Deziel MR, Parsons LM, Salfinger M, Drusano GL. Selection of a moxifloxacin dose that suppresses drug resistance in Mycobacterium tuberculosis, by use of an in vitro pharmacodynamic infection model and mathematical modeling. $J$ Infect Dis 2004;190:1642-1651.

177. Brunton L. Knollmann B, Hilal-Dandan R. Goodman \& Gilman's the pharmacological basis of therapeutics. New York: McGraw Hill Medical; 2018.

178. Kahlmeter G, Dahlager JI. Aminoglycoside toxicity - a review of clinical studies published between 1975 and 1982. J Antimicrob Chemother 1984;13:9-22.

179. Arnold A, Cooke GS, Kon OM, Dedicoat M, Lipman M, Loyse A, et al. Adverse effects and choice between the injectable agents amikacin and capreomycin in multidrug-resistant tuberculosis. Antimicrob Agents Chemother 2017;61:e02586-16.

180. Sturdy A, Goodman A, José RJ, Loyse A, O'Donoghue M, Kon OM, et al. Multidrug-resistant tuberculosis (MDR-TB) treatment in the UK: a study of injectable use and toxicity in practice. $J$ Antimicrob Chemother 2011;66:1815-1820.

181. Sagwa EL, Ruswa N, Mavhunga F, Rennie T, Leufkens HG, MantelTeeuwisse AK. Comparing amikacin and kanamycin-induced hearing loss in multidrug-resistant tuberculosis treatment under programmatic conditions in a Namibian retrospective cohort. BMC Pharmacol Toxicol 2015;16:36.

182. Seddon JA, Thee S, Jacobs K, Ebrahim A, Hesseling AC, Schaaf HS. Hearing loss in children treated for multidrug-resistant tuberculosis. $J$ Infect 2013;66:320-329.

183. Garcia-Prats AJ, Rose PC, Draper HR, Seddon JA, Norman J, Mclleron HM, et al. Effect of coadministration of lidocaine on the pain and pharmacokinetics of intramuscular amikacin in children with multidrug-resistant tuberculosis: a randomized crossover trial. Pediatr Infect Dis J 2018;37:1199-1203.

184. Bardien S, Human H, Harris T, Hefke G, Veikondis R, Schaaf HS, et al. A rapid method for detection of five known mutations associated with aminoglycoside-induced deafness. BMC Med Genet 2009;10:2.

185. Selimoglu E. Aminoglycoside-induced ototoxicity. Curr Pharm Des 2007;13:119-126.

186. Fischel-Ghodsian N. Genetic factors in aminoglycoside toxicity. Pharmacogenomics 2005;6:27-36.

187. Kranzer K, Elamin WF, Cox H, Seddon JA, Ford N, Drobniewski F. A systematic review and meta-analysis of the efficacy and safety of $\mathrm{N}$-acetylcysteine in preventing aminoglycoside-induced ototoxicity: implications for the treatment of multidrug-resistant TB. Thorax 2015;70:1070-1077.

188. Perletti G, Vral A, Patrosso MC, Marras E, Ceriani I, Willems P, et al. Prevention and modulation of aminoglycoside ototoxicity (review). Mol Med Rep 2008;1:3-13.

189. Livermore DM. Linezolid in vitro: mechanism and antibacterial spectrum. J Antimicrob Chemother 2003;51:ii9-ii16.

190. Beekmann SE, Gilbert DN, Polgreen PM; IDSA Emerging Infections Network. Toxicity of extended courses of linezolid: results of an Infectious Diseases Society of America Emerging Infections Network survey. Diagn Microbiol Infect Dis 2008;62: 407-410.

191. Soriano A, Miró O, Mensa J. Mitochondrial toxicity associated with linezolid. N Engl J Med 2005;353:2305-2306.

192. Migliori GB, Eker B, Richardson MD, Sotgiu G, Zellweger JP, Skrahina A, et al.; TBNET Study Group. A retrospective TBNET assessment of linezolid safety, tolerability and efficacy in multidrug-resistant tuberculosis. Eur Respir J 2009;34:387-393.

193. Sotgiu G, Pontali E, Migliori GB. Linezolid to treat MDR-/XDRtuberculosis: available evidence and future scenarios. Eur Respir $J$ 2015;45:25-29.

194. Sotgiu G, Centis R, D'Ambrosio L, Spanevello A, Migliori GB; International Group for the study of Linezolid. Linezolid to treat extensively drug-resistant TB: retrospective data are confirmed by experimental evidence. Eur Respir J 2013;42: 288-290.

195. Lee M, Lee J, Carroll MW, Choi H, Min S, Song T, et al. Linezolid for treatment of chronic extensively drug-resistant tuberculosis. $N$ Engl J Med 2012;367:1508-1518.
196. Sotgiu G, Centis R, D'Ambrosio L, Alffenaar JW, Anger HA, Caminero $\mathrm{JA}$, et al. Efficacy, safety and tolerability of linezolid containing regimens in treating MDR-TB and XDR-TB: systematic review and meta-analysis. Eur Respir J 2012;40:1430-1442.

197. Kamp J, Bolhuis MS, Tiberi S, Akkerman OW, Centis R, de Lange WC, et al. Simple strategy to assess linezolid exposure in patients with multi-drug-resistant and extensively-drug-resistant tuberculosis. Int $J$ Antimicrob Agents 2017;49:688-694.

198. Bolhuis MS, Tiberi S, Sotgiu G, De Lorenzo S, Kosterink JG, van der Werf TS, et al. Is there still room for therapeutic drug monitoring of linezolid in patients with tuberculosis? Eur Respir J 2016;47: 1288-1290.

199. Garcia-Prats AJ, Rose PC, Hesseling AC, Schaaf HS. Linezolid for the treatment of drug-resistant tuberculosis in children: a review and recommendations. Tuberculosis (Edinb) 2014;94:93-104.

200. Prieto LM, Santiago B, Del Rosal T, Carazo B, Jimenez AB, PerezGorricho B, et al. Spanish Paediatric TBRN. Linezolid-containing treatment regimens for tuberculosis in children. Pediatr Infect Dis $J$ 2019;38:263-267.

201. Nagiec EE, Wu L, Swaney SM, Chosay JG, Ross DE, Brieland JK, et al. Oxazolidinones inhibit cellular proliferation via inhibition of mitochondrial protein synthesis. Antimicrob Agents Chemother 2005;49:3896-3902.

202. Bolhuis MS, Akkerman OW, Sturkenboom MGG, Ghimire S, Srivastava S, Gumbo T, et al. Linezolid-based regimens for multidrug-resistant tuberculosis (TB): a systematic review to establish or revise the current recommended dose for TB treatment. Clin Infect Dis 2018;67:S327-S335.

203. Srivastava S, Magombedze G, Koeuth T, Sherman C, Pasipanodya JG, Raj $P$, et al. Linezolid dose that maximizes sterilizing effect while minimizing toxicity and resistance emergence for tuberculosis. Antimicrob Agents Chemother 2017;61:e00751-17.

204. Song T, Lee M, Jeon HS, Park Y, Dodd LE, Dartois V, et al. Linezolid trough concentrations correlate with mitochondrial toxicity-related adverse events in the treatment of chronic extensively drugresistant tuberculosis. EBioMedicine 2015;2:1627-1633.

205. Vinh DC, Rubinstein E. Linezolid: a review of safety and tolerability. $J$ Infect 2009;59:S59-S74.

206. Griffith DE, Aksamit T, Brown-Elliott BA, Catanzaro A, Daley C, Gordin F, et al.; ATS Mycobacterial Diseases Subcommittee; American Thoracic Society; Infectious Disease Society of America. An official ATS/IDSA statement: diagnosis, treatment, and prevention of nontuberculous mycobacterial diseases. Am J Respir Crit Care Med 2007;175:367-416. [Published erratum appears in Am J Respir Crit Care Med 175:744-745.]

207. Andini N, Nash KA. Intrinsic macrolide resistance of the Mycobacterium tuberculosis complex is inducible. Antimicrob Agents Chemother 2006;50:2560-2562.

208. Nash KA. Intrinsic macrolide resistance in Mycobacterium smegmatis is conferred by a novel erm gene, erm(38). Antimicrob Agents Chemother 2003;47:3053-3060.

209. Truffot-Pernot C, Lounis N, Grosset JH, Ji B. Clarithromycin is inactive against Mycobacterium tuberculosis. Antimicrob Agents Chemother 1995;39:2827-2828.

210. Bolhuis MS, van Altena R, van Soolingen D, de Lange WC, Uges DR, van der Werf TS, et al. Clarithromycin increases linezolid exposure in multidrug-resistant tuberculosis patients. Eur Respir J 2013;42: 1614-1621.

211. van der Paardt AF, Wilffert B, Akkerman OW, de Lange WC, van Soolingen D, Sinha B, et al. Evaluation of macrolides for possible use against multidrug-resistant Mycobacterium tuberculosis. Eur Respir J 2015;46:444-455.

212. Lehmann J. Para-aminosalicylic acid in the treatment of tuberculosis. Lancet 1946;1:15.

213. Zheng J, Rubin EJ, Bifani P, Mathys V, Lim V, Au M, et al. paraAminosalicylic acid is a prodrug targeting dihydrofolate reductase in Mycobacterium tuberculosis. J Biol Chem 2013;288: 23447-23456.

214. Shane SJ, Laurie JH, Riley C, Boutilier M. Effect of combined therapy (dihydrostreptomycin and PAS) on the emergence of streptomycinresistant strains of tubercle bacilli. N Engl J Med 1952;246: 132-134. 
215. Friedman B, Sale L Jr, Goldman A. The effect of para-aminosalicylic acid (PAS) on the development of resistance of tubercle bacilli to streptomycin. Am J Med Sci 1952;224:53-56.

216. Donald PR, Diacon AH. Para-aminosalicylic acid: the return of an old friend. Lancet Infect Dis 2015;15:1091-1099.

217. Zignol M, Cabibbe AM, Dean AS, Glaziou P, Alikhanova N, Ama C, et al. Genetic sequencing for surveillance of drug resistance in tuberculosis in highly endemic countries: a multi-country population-based surveillance study. Lancet Infect Dis 2018;18: 675-683.

218. Zignol M, Dean AS, Alikhanova N, Andres S, Cabibbe AM, Cirillo DM, et al. Population-based resistance of Mycobacterium tuberculosis isolates to pyrazinamide and fluoroquinolones: results from a multicountry surveillance project. Lancet Infect Dis 2016;16: 1185-1192.

219. Velásquez GE, Calderon RI, Mitnick CD, Becerra MC, Huang CC, Zhang Z, et al. Pyrazinamide resistance assays and two-month sputum culture status in patients with multidrug-resistant tuberculosis. Antimicrob Agents Chemother 2016;60: 6766-6773.

220. Njire M, Tan Y, Mugweru J, Wang C, Guo J, Yew W, et al. Pyrazinamide resistance in Mycobacterium tuberculosis: review and update. Adv Med Sci 2016;61:63-71.

221. Cabibbe AM, Sotgiu G, Izco S, Migliori GB. Genotypic and phenotypic M. tuberculosis resistance: guiding clinicians to prescribe the correct regimens. Eur Respir J 2017;50:1702292.

222. Miotto $P$, Tessema B, Tagliani E, Chindelevitch L, Starks AM, Emerson $\mathrm{C}$, et al. A standardised method for interpreting the association between mutations and phenotypic drug resistance in Mycobacterium tuberculosis. Eur Respir J 2017;50:1701354.

223. Fox W, Ellard GA, Mitchison DA. Studies on the treatment of tuberculosis undertaken by the british medical research council tuberculosis units, 1946-1986, with relevant subsequent publications. Int J Tuberc Lung Dis 1999;3:S231-S279.

224. Steele MA, Des Prez RM. The role of pyrazinamide in tuberculosis chemotherapy. Chest 1988;94:845-850.

225. Saukkonen JJ, Cohn DL, Jasmer RM, Schenker S, Jereb JA, Nolan CM, et al.; ATS (American Thoracic Society) Hepatotoxicity of Antituberculosis Therapy Subcommittee. An official ATS statement: hepatotoxicity of antituberculosis therapy. Am J Respir Crit Care Med 2006;174:935-952.

226. Bastos ML, Lan Z, Menzies D. An updated systematic review and meta-analysis for treatment of multidrug-resistant tuberculosis. Eur Respir J 2017;49:1600803.

227. Saukkonen JJ, Powell K, Jereb JA. Monitoring for tuberculosis drug hepatotoxicity: moving from opinion to evidence. Am J Respir Crit Care Med 2012;185:598-599.

228. Zignol M, Floyd K. Resistance of Mycobacterium tuberculosis isolates to pyrazinamide and fluoroquinolones: authors' reply. Lancet Infect Dis 2017;17:25.

229. Zignol M, Dean AS, Falzon D, van Gemert W, Wright A, van Deun A et al. Twenty years of global surveillance of antituberculosis-drug resistance. N Engl J Med 2016;375:1081-1089.

230. Allix-Béguec C, Arandjelovic I, Bi L, Beckert P, Bonnet M, Bradley P, et al.; CRyPTIC Consortium and the 100,000 Genomes Project. Prediction of susceptibility to first-line tuberculosis drugs by DNA sequencing. N Engl J Med 2018:379:1403-1415.

231. Falzon D, Jaramillo E, Gilpin C, Weyer K. The role of novel approaches and new findings in the pharmacology of tuberculosis medicines in improving treatment outcomes. Clin Infect Dis 2018; 67:S365-S367.

232. de Miranda Silva C, Hajihosseini A, Myrick J, Nole J, Louie A, Schmidt $\mathrm{S}$, et al. Effect of moxifloxacin plus pretomanid against Mycobacterium tuberculosis in log phase, acid phase, and nonreplicating-persister phase in an in vitro assay. Antimicrob Agents Chemother 2018;63:e01695-18.

233. de Miranda Silva C, Hajihosseini A, Myrick J, Nole J, Louie A, Schmidt $\mathrm{S}$, et al. Effect of linezolid plus bedaquiline against mycobacterium tuberculosis in log phase, acid phase, and nonreplicating-persister phase in an in vitro assay. Antimicrob Agents Chemother 2018;62: e00856-18.
234. Ammerman NC, Swanson RV, Bautista EM, Almeida DV, Saini V, Omansen TF, et al. Impact of clofazimine dosing on treatment shortening of the first-line regimen in a mouse model of tuberculosis. Antimicrob Agents Chemother 2018;62: e00636-18.

235. Tucker EW, Guglieri-Lopez B, Ordonez AA, Ritchie B, Klunk MH, Sharma $\mathrm{R}$, et al. Noninvasive ${ }^{11} \mathrm{C}$-rifampin positron emission tomography reveals drug biodistribution in tuberculous meningitis. Sci Transl Med 2018;10:eaau0965.

236. Zimmerman M, Blanc L, Chen PY, Dartois V, Prideaux B. Spatial quantification of drugs in pulmonary tuberculosis lesions by laser capture microdissection liquid chromatography mass spectrometry (LCM-LC/MS). J Vis Exp 2018;(134):10.3791/57402.

237. Via LE, England K, Weiner DM, Schimel D, Zimmerman MD, Dayao $\mathrm{E}$, et al. A sterilizing tuberculosis treatment regimen is associated with faster clearance of bacteria in cavitary lesions in marmosets. Antimicrob Agents Chemother 2015;59:4181-4189.

238. Chideya S, Winston CA, Peloquin CA, Bradford WZ, Hopewell PC, Wells CD, et al. Isoniazid, rifampin, ethambutol, and pyrazinamide pharmacokinetics and treatment outcomes among a predominantly HIV-infected cohort of adults with tuberculosis from Botswana. Clin Infect Dis 2009;48:1685-1694.

239. Savic RM, Weiner M, MacKenzie WR, Engle M, Whitworth WC, Johnson JL, et al.; Tuberculosis Trials Consortium of the Centers for Disease Control and Prevention. Defining the optimal dose of rifapentine for pulmonary tuberculosis: exposure-response relations from two phase II clinical trials. Clin Pharmacol Ther 2017; 102:321-331.

240. Svensson EM, Svensson RJ, Te Brake LHM, Boeree MJ, Heinrich N, Konsten S, et al. The potential for treatment shortening with higher rifampicin doses: relating drug exposure to treatment response in patients with pulmonary tuberculosis. Clin Infect Dis 2018;67:34-41.

241. Alsultan A, Peloquin CA. Therapeutic drug monitoring in the treatment of tuberculosis: an update. Drugs 2014;74:839-854. [Published erratum appears in Drugs 74:2061.]

242. Peloquin $\mathrm{C}$. The role of therapeutic drug monitoring in mycobacteria infections. Microbiol Spectr 2017:5:10.1128/microbiolspec.TNMI70029-2016.

243. Gumbo T, Alffenaar JC. Pharmacokinetic/pharmacodynamic background and methods and scientific evidence base for dosing of second-line tuberculosis drugs. Clin Infect Dis 2018;67: S267-S273.

244. Lange C, Alghamdi WA, Al-Shaer MH, Brighenti S, Diacon AH, DiNardo AR, et al. Perspectives for personalized therapy for patients with multidrug-resistant tuberculosis. J Intern Med [online ahead of print] 28 May 2018; DOI: 10.1111/joim.12780.

245. van Altena R, Dijkstra JA, van der Meer ME, Borjas Howard JF, Kosterink JG, van Soolingen D, et al. Reduced chance of hearing loss associated with therapeutic drug monitoring of aminoglycosides in the treatment of multidrugresistant tuberculosis. Antimicrob Agents Chemother 2017; 61:e01400-16.

246. Deshpande D, Alffenaar JC, Köser CU, Dheda K, Chapagain ML, Simbar N, et al. D-cycloserine pharmacokinetics/pharmacodynamics, susceptibility, and dosing implications in multidrug-resistant tuberculosis: a Faustian deal. Clin Infect Dis 2018;67:S308-S316.

247. Brown AN, Drusano GL, Adams JR, Rodriquez JL, Jambunathan K, Baluya DL, et al. Preclinical evaluations to identify optimal linezolid regimens for tuberculosis therapy. MBio 2015;6:e01741-e15.

248. Pranger AD, van der Werf TS, Kosterink JGW, Alffenaar JWC. The role of fluoroquinolones in the treatment of tuberculosis in 2019. Drugs 2019;79:161-171.

249. Ahmad KF, Hamid S, du Cros P, Casas E, Khamraev A, Sikhondze W, et al. Effectiveness and safety of standardised shorter regimens for multidrug-resistant tuberculosis: individual patient data and aggregate data meta-analyses. Eur Respir J 2017;50:1700061.

250. Trébucq A, Schwoebel V, Kashongwe Z, Bakayoko A, Kuaban C, Noeske J, et al. Treatment outcome with a short multidrug-resistant tuberculosis regimen in nine African countries. Int $J$ Tuberc Lung Dis 2018;22:17-25. 
251. Gama E, Madan J, Langley I, Girma M, Evans D, Rosen S, et al. Economic evaluation of a shortened standardised treatment regimen of antituberculosis drugs for patients with multidrugresistant tuberculosis (STREAM): study protocol. BMJ Open 2016; 6:e014386.

252. Akkermans R. 48th Union World Conference on Lung Health. Lancet Respir Med 2017;5:926-927.

253. World Health Organization. WHO position statement on the shorter MDR-TB regimen. Licence: Cc by-nc-sa 3.0 igo. 2018 [accessed 2019 Sep 15]. Available from: https://www.who.int/tb/areas-ofwork/drug-resistant-tb/treatment/WHOPositionStatement ShorterRegimensSTREAMStage1.pdf?ua $=1$.

254. Lange C, Duarte R, Fréchet-Jachym M, Guenther G, Guglielmetti L, Olaru ID, et al.; European MDR-TB database collaboration. Limited benefit of the new shorter multidrug-resistant tuberculosis regimen in Europe. Am J Respir Crit Care Med 2016;194: 1029-1031.

255. Munoz-Torrico M, Salazar M, Millan M, Martínez OJA, Narvaez DLA, Segura Del Pilar M. Eligibility for the shorter regimen for multidrug-resistant tuberculosis in Mexico. Eur Respir J 2018;50: 1702267.

256. Tsang CA, Shah N, Armstrong LR, Marks SM. Eligibility for a shorter treatment regimen for multidrug-resistant tuberculosis in the United States, 2011-2016. Clin Infect Dis 2019;ciz263.

257. Barry PM, Lowenthal P, True L, Henry L, Schack G, Wendorf K, et al. Benefit of the shorter multidrug-resistant tuberculosis treatment regimen in California and modified eligibility criteria. Am J Respir Crit Care Med 2017;196:1488-1489.

258. Seifert M, Catanzaro D, Catanzaro A, Rodwell TC. Genetic mutations associated with isoniazid resistance in Mycobacterium tuberculosis: a systematic review. PLoS One 2015;10:e0119628.

259. Van Deun A, Chiang CY. Shortened multidrug-resistant tuberculosis regimens overcome low-level fluoroquinolone resistance. Eur Respir J 2017;49:1700223.

260. Horne DJ, Pinto LM, Arentz M, Lin SY, Desmond E, Flores LL, et al. Diagnostic accuracy and reproducibility of WHO-endorsed phenotypic drug susceptibility testing methods for first-line and second-line antituberculosis drugs. J Clin Microbiol 2013;51:393-401.

261. Angra PK, Taylor TH, lademarco MF, Metchock B, Astles JR, Ridderhof JC. Performance of tuberculosis drug susceptibility testing in U.S. laboratories from 1994 to 2008. J Clin Microbiol 2012;50:1233-1239.

262. Resist-TB. DR-TB clinical trials progress report; 2018 [accessed 2018 Jan 19]. Available from: http://www.resisttb.org/?page_id=1602.

263. Marrone MT, Venkataramanan V, Goodman M, Hill AC, Jereb JA, Mase SR. Surgical interventions for drug-resistant tuberculosis: a systematic review and meta-analysis. Int J Tuberc Lung Dis 2013;17:6-16.

264. Naidoo R. Surgery for pulmonary tuberculosis. Curr Opin Pulm Med 2008;14:254-259.

265. Pomerantz BJ, Cleveland JC Jr, Olson HK, Pomerantz M. Pulmonary resection for multi-drug resistant tuberculosis. J Thorac Cardiovasc Surg 2001;121:448-453.

266. Pomerantz M, Mault JR. History of resectional surgery for tuberculosis and other mycobacterial infections. Chest Surg Clin N Am 2000;10: 131-133, ix.

267. World Health Organization. The role of surgery in the treatment of pulmonary tb and multidrug-and extensively drug-resistant tb. Geneva, Switzerland: World Health Organization; 2014.

268. Odell JA. The history of surgery for pulmonary tuberculosis. Thorac Surg Clin 2012;22:257-269.

269. Alexander GR, Biccard B. A retrospective review comparing treatment outcomes of adjuvant lung resection for drug-resistant tuberculosis in patients with and without human immunodeficiency virus co-infection. Eur $J$ Cardiothorac Surg 2016;49:823-828.

270. Dara M, Sotgiu G, Zaleskis R, Migliori GB. Untreatable tuberculosis: is surgery the answer? Eur Respir J 2015;45:577-582.

271. Fox GJ, Mitnick CD, Benedetti A, Chan ED, Becerra M, Chiang CY, et al.; Collaborative Group for Meta-Analysis of Individual Patient Data in MDR-TB. Surgery as an adjunctive treatment for multidrug- resistant tuberculosis: an individual patient data metaanalysis. Clin Infect Dis 2016;62:887-895.

272. Harris RC, Khan MS, Martin LJ, Allen V, Moore DA, Fielding K, et al.; LSHTM MDR-TB surgery systematic review group. The effect of surgery on the outcome of treatment for multidrug-resistant tuberculosis: a systematic review and meta-analysis. BMC Infect Dis 2016;16:262.

273. Borisov SE, D'Ambrosio L, Centis R, Tiberi S, Dheda K, Alffenaar JW, et al. Outcomes of patients with drug-resistant-tuberculosis treated with bedaquiline-containing regimens and undergoing adjunctive surgery. J Infect 2019;78:35-39.

274. Gegia M, Winters N, Benedetti A, van Soolingen D, Menzies D. Treatment of isoniazid-resistant tuberculosis with first-line drugs: a systematic review and meta-analysis. Lancet Infect Dis 2017;17:223-234.

275. Blumberg HM, Burman WJ, Chaisson RE, Daley CL, Etkind SC, Friedman LN, et al.; American Thoracic Society, Centers for Disease Control and Prevention and the Infectious Diseases Society. American Thoracic Society/Centers for Disease Control and Prevention/Infectious Diseases Society of America: treatment of tuberculosis. Am J Respir Crit Care Med 2003;167: 603-662.

276. Fregonese F, Ahuja SD, Akkerman OW, Arakaki-Sanchez D, Ayakaka I, Baghaei $\mathrm{P}$, et al. Comparison of different treatments for isoniazidresistant tuberculosis: an individual patient data meta-analysis. Lancet Respir Med 2018;6:265-275.

277. Yee D, Valiquette C, Pelletier M, Parisien I, Rocher I, Menzies D. Incidence of serious side effects from first-line antituberculosis drugs among patients treated for active tuberculosis. Am J Respir Crit Care Med 2003;167:1472-1477.

278. Weiner M, Burman W, Luo CC, Peloquin CA, Engle M, Goldberg S, et al. Effects of rifampin and multidrug resistance gene polymorphism on concentrations of moxifloxacin. Antimicrob Agents Chemother 2007;51:2861-2866.

279. Nijland HM, Ruslami R, Suroto AJ, Burger DM, Alisjahbana B, van Crevel $\mathrm{R}$, et al. Rifampicin reduces plasma concentrations of moxifloxacin in patients with tuberculosis. Clin Infect Dis 2007;45: 1001-1007.

280. Vernon A, Burman W, Benator D, Khan A, Bozeman L. Acquired rifamycin monoresistance in patients with HIV-related tuberculosis treated with once-weekly rifapentine and isoniazid: Tuberculosis Trials Consortium. Lancet 1999;353:1843-1847.

281. Lutfey M, Della-Latta P, Kapur V, Palumbo LA, Gurner D, Stotzky G, et al. Independent origin of mono-rifampin-resistant Mycobacterium tuberculosis in patients with AIDS. Am J Respir Crit Care Med 1996; 153:837-840.

282. Isaakidis P, Casas EC, Das M, Tseretopoulou X, Ntzani EE, Ford N. Treatment outcomes for HIV and MDR-TB co-infected adults and children: systematic review and meta-analysis. Int J Tuberc Lung Dis 2015;19:969-978.

283. Gandhi NR, Andrews JR, Brust JC, Montreuil R, Weissman D, Heo M, et al. Risk factors for mortality among MDR- and XDR-TB patients in a high HIV prevalence setting. Int J Tuberc Lung Dis 2012;16:90-97.

284. Marks SM, Flood J, Seaworth B, Hirsch-Moverman Y, Armstrong L, Mase S, et al.; TB Epidemiologic Studies Consortium. Treatment practices, outcomes, and costs of multidrug-resistant and extensively drug-resistant tuberculosis, United States, 2005-2007. Emerg Infect Dis 2014;20:812-821.

285. World Health Organization. WHO policy on collaborative TB/HIV activities: guidelines for national programmes and other stakeholders. Geneva, Switzerland: World Health Organization; 2012.

286. Blanc FX, Sok T, Laureillard D, Borand L, Rekacewicz C, Nerrienet E, et al.; CAMELIA (ANRS 1295-CIPRA KH001) Study Team. Earlier versus later start of antiretroviral therapy in HIV-infected adults with tuberculosis. N Engl J Med 2011;365:1471-1481.

287. Abdool Karim SS, Naidoo K, Grobler A, Padayatchi N, Baxter C, Gray $\mathrm{AL}$, et al. Integration of antiretroviral therapy with tuberculosis treatment. N Engl J Med 2011;365:1492-1501.

288. Abdool Karim SS, Naidoo K, Grobler A, Padayatchi N, Baxter C, Gray $A$, et al. Timing of initiation of antiretroviral drugs during tuberculosis therapy. N Engl J Med 2010;362:697-706. 
289. Masur H, Brooks JT, Benson CA, Holmes KK, Pau AK, Kaplan JE; National Institutes of Health; Centers for Disease Control and Prevention; HIV Medicine Association of the Infectious Diseases Society of America. Prevention and treatment of opportunistic infections in HIV-infected adults and adolescents: updated guidelines from the Centers for Disease Control and Prevention, National Institutes of Health, and HIV Medicine Association of the Infectious Diseases Society of America. Clin Infect Dis 2014;58: 1308-1311.

290. Padayatchi N, Abdool Karim SS, Naidoo K, Grobler A, Friedland G. Improved survival in multidrug-resistant tuberculosis patients receiving integrated tuberculosis and antiretroviral treatment in the SAPiT Trial. Int J Tuberc Lung Dis 2014;18: 147-154.

291. Palacios E, Franke M, Muñoz M, Hurtado R, Dallman R, Chalco K, et al. HIV-positive patients treated for multidrug-resistant tuberculosis: clinical outcomes in the HAART era. Int J Tuberc Lung Dis 2012;16:348-354. [Published erratum appears in Int J Tuberc Lung Dis 16:568.]

292. Arentz M, Pavlinac P, Kimerling ME, Horne DJ, Falzon D, Schünemann HJ, et al.; ART study group. Use of anti-retroviral therapy in tuberculosis patients on second-line anti-TB regimens: a systematic review. PLoS One 2012;7:e47370.

293. Dheda K, Shean K, Zumla A, Badri M, Streicher EM, Page-Shipp $\mathrm{L}$, et al. Early treatment outcomes and HIV status of patients with extensively drug-resistant tuberculosis in South Africa: a retrospective cohort study. Lancet 2010;375:17981807.

294. O’Donnell MR, Padayatchi N, Kvasnovsky C, Werner L, Master I, Horsburgh CR Jr. Treatment outcomes for extensively drugresistant tuberculosis and HIV co-infection. Emerg Infect Dis 2013; 19:416-424.

295. Kvasnovsky CL, Cegielski JP, Erasmus R, Siwisa NO, Thomas K, der Walt ML. Extensively drug-resistant TB in Eastern Cape, South Africa: high mortality in HIV-negative and HIV-positive patients. $J$ Acquir Immune Defic Syndr 2011;57:146-152.

296. Satti H, McLaughlin MM, Hedt-Gauthier B, Atwood SS, Omotayo DB, Ntlamelle L, et al. Outcomes of multidrug-resistant tuberculosis treatment with early initiation of antiretroviral therapy for HIV co-infected patients in Lesotho. PLoS One 2012;7:e46943.

297. Pietersen E, Ignatius E, Streicher EM, Mastrapa B, Padanilam X, Pooran A, et al. Long-term outcomes of patients with extensively drug-resistant tuberculosis in South Africa: a cohort study. Lancet 2014;383:1230-1239.

298. Vinnard C, Winston CA, Wileyto EP, Macgregor RR, Bisson GP. Isoniazid resistance and death in patients with tuberculous meningitis: retrospective cohort study. BMJ 2010;341:c4451.

299. Tho DQ, Török ME, Yen NT, Bang ND, Lan NT, Kiet VS, et al. Influence of antituberculosis drug resistance and Mycobacterium tuberculosis lineage on outcome in HIVassociated tuberculous meningitis. Antimicrob Agents Chemother 2012;56:3074-3079.

300. Vinnard C, King L, Munsiff S, Crossa A, Iwata K, Pasipanodya J, et al. Long-term mortality of patients with tuberculous meningitis in New York City: a cohort study. Clin Infect Dis 2017;64:401-407.

301. Havlir DV, Kendall MA, Ive P, Kumwenda J, Swindells S, Qasba SS, et al.; AIDS Clinical Trials Group Study A5221. Timing of antiretroviral therapy for HIV-1 infection and tuberculosis. N Engl J Med 2011;365:1482-1491.

302. Török ME, Yen NT, Chau TT, Mai NT, Phu NH, Mai PP, et al. Timing of initiation of antiretroviral therapy in human immunodeficiency virus (HIV): associated tuberculous meningitis. Clin Infect Dis 2011;52: 1374-1383.

303. Mallikaarjun S, Wells C, Petersen C, Paccaly A, Shoaf SE, Patil S, et al. Delamanid coadministered with antiretroviral drugs or antituberculosis drugs shows no clinically relevant drug-drug interactions in healthy subjects. Antimicrob Agents Chemother 2016;60:5976-5985.

304. Kitahata MM, Koepsell TD, Deyo RA, Maxwell CL, Dodge WT, Wagner EH. Physicians' experience with the acquired immunodeficiency syndrome as a factor in patients' survival. N Engl J Med 1996;334: 701-706.
305. Thompson MA, Mugavero MJ, Amico KR, Cargill VA, Chang LW, Gross $\mathrm{R}$, et al. Guidelines for improving entry into and retention in care and antiretroviral adherence for persons with HIV: evidencebased recommendations from an International Association of Physicians in AIDS Care panel. Ann Intern Med 2012;156:817-833, W-284, W-285, W-286, W-287, W-288, W-289, W-290, W-291, W-292, W-293, W-294.

306. Dodd PJ, Gardiner E, Coghlan R, Seddon JA. Burden of childhood tuberculosis in 22 high-burden countries: a mathematical modelling study. Lancet Glob Health 2014;2:e453-e459.

307. Jenkins HE, Yuen CM, Rodriguez CA, Nathavitharana RR, McLaughlin $\mathrm{MM}$, Donald $\mathrm{P}$, et al. Mortality in children diagnosed with tuberculosis: a systematic review and meta-analysis. Lancet Infect Dis 2017;17:285-295.

308. Dodd PJ, Sismanidis C, Seddon JA. Global burden of drug-resistant tuberculosis in children: a mathematical modelling study. Lancet Infect Dis 2016;16:1193-1201.

309. Jenkins HE, Tolman AW, Yuen CM, Parr JB, Keshavjee S, Pérez-Vélez $\mathrm{CM}$, et al. Incidence of multidrug-resistant tuberculosis disease in children: systematic review and global estimates. Lancet 2014;383: 1572-1579.

310. Garcia-Prats AJ, Schaaf HS, Draper HR, Garcia-Cremades M, Winckler J, Wiesner L, et al. Pharmacokinetics, safety and optimal dosing of linezolid in children with multidrug-resistant tuberculosis. Int J Tuberc Lung Dis 2018;22:S593.

311. Schaaf HS, Garcia-Prats AJ, Hesseling AC, Seddon JA. Managing multidrug-resistant tuberculosis in children: review of recent developments. Curr Opin Infect Dis 2014;27:211-219.

312. Bothamley GH, Ehlers C, Salonka I, Skrahina A, Orcau A, Codecasa LR, et al. Pregnancy in patients with tuberculosis: a TBNET crosssectional survey. BMC Pregnancy Childbirth 2016;16:304.

313. K DV, A M, S R, K AV. Multidrug-resistant tuberculosis in pregnancy. J Coll Physicians Surg Pak 2007;17:637-639.

314. Oliveira HB, Mateus SH. Characterization of multidrug-resistant tuberculosis during pregnancy in Campinas, State of São Paulo, Brazil, from 1995 to 2007 [in Portuguese]. Rev Soc Bras Med Trop 2011;44:627-630.

315. Palacios E, Dallman R, Muñoz M, Hurtado R, Chalco K, Guerra D, et al. Drug-resistant tuberculosis and pregnancy: treatment outcomes of 38 cases in Lima, Peru. Clin Infect Dis 2009;48:1413-1419.

316. Shin S, Guerra D, Rich M, Seung KJ, Mukherjee J, Joseph K, et al. Treatment of multidrug-resistant tuberculosis during pregnancy: a report of 7 cases. Clin Infect Dis 2003:36:996-1003.

317. Tabarsi P, Baghaei P, Mirsaeidi M, Amiri M, Mansouri D, Novin A, et al. Multi-drug resistant tuberculosis in pregnancy: need for more intensive treatment. Infection 2007;35:477-478.

318. Tabarsi P, Moradi A, Baghaei P, Marjani M, Shamaei M, Mansouri N, et al. Standardised second-line treatment of multidrug-resistant tuberculosis during pregnancy. Int J Tuberc Lung Dis 2011;15:547-550.

319. Takashima T, Danno K, Tamura Y, Nagai T, Matsumoto T, Han Y, et al. Treatment outcome of patients with multidrug-resistant pulmonary tuberculosis during pregnancy [in Japanese]. Kekkaku 2006;81: 413-418.

320. Jaspard M, Elefant-Amoura E, Melonio I, De Montgolfier I, Veziris N, Caumes E. Bedaquiline and linezolid for extensively drug-resistant tuberculosis in pregnant woman. Emerg Infect Dis 2017;23: 10.3201/eid2310.161398.

321. Lessnau KD, Qarah S. Multidrug-resistant tuberculosis in pregnancy: case report and review of the literature. Chest 2003;123:953-956.

322. Nitta AT, Milligan D. Management of four pregnant women with multidrug-resistant tuberculosis. Clin Infect Dis 1999;28: 1298-1304.

323. Ünlü M, Çimen P, Arı G, Șevket Dereli M. A successfully treated severe case of extensively drug-resistant tuberculosis during pregnancy. Respir Case Rep 2015;4:67-71.

324. Mukherjee JS, Shin S, Furin J, Rich ML, Léandre F, Joseph JK, et al. New challenges in the clinical management of drug-resistant tuberculosis. Infect Dis Clin Pract 2002;11:329-339.

325. Khan M, Pillay T, Moodley J, Ramjee A, Padayatchi N. Pregnancies complicated by multidrug-resistant tuberculosis and HIV coinfection in Durban, South Africa. Int J Tuberc Lung Dis 2007;11: 706-708. 
326. Rohilla M, Joshi B, Jain V, Kalra J, Prasad GR. Multidrug-resistant tuberculosis during pregnancy: two case reports and review of the literature. Case Rep Obstet Gynecol 2016;2016:1536281.

327. Van Kampenhout E, Bolhuis MS, Alffenaar JC, Oswald LM, Kerstjens $\mathrm{HA}$, de Lange WC, et al. Pharmacokinetics of moxifloxacin and linezolid during and after pregnancy in a patient with multidrugresistant tuberculosis. Eur Respir J 2017;49:1601724.

328. U.S. Food and Drug Administration. Pregnancy, lactation, and reproductive potential: labeling for human prescription drug and biological products-content and format. Guidance for industry; 2014 [accessed 2019 Feb 8]. Available from: https://www.fda.gov/downloads/Drugs/ GuidanceComplianceRegulatoryInformation/Guidances/ UCM425398.pdf.

329. Esmail A, Sabur NF, Okpechi I, Dheda K. Management of drug-resistant tuberculosis in special sub-populations including those with HIV coinfection, pregnancy, diabetes, organ-specific dysfunction, and in the critically ill. J Thorac Dis 2018;10:3102-3118.

330. Gupta A, Mathad JS, Abdel-Rahman SM, Albano JD, Botgros R, Brown V, et al. Toward earlier inclusion of pregnant and postpartum women in tuberculosis drug trials: consensus statements from an international expert panel. Clin Infect Dis 2016;62:761-769.

331. American Thoracic Society. Targeted tuberculin testing and treatment of latent tuberculosis infection. MMWR Recomm Rep 2000;49:1-51.

332. Management of persons exposed to multidrug-resistant tuberculosis. MMWR Recomm Rep 1992;41:61-71.

333. Marks SM, Mase SR, Morris SB. Systematic review, meta-analysis, and cost-effectiveness of treatment of latent tuberculosis to reduce progression to multidrug-resistant tuberculosis. Clin Infect Dis 2017;64:1670-1677.

334. Seddon J, Fred D, Amanullah F, Schaaf H, Starke J, Keshavjee S, et al. Post-exposure management of multidrug-resistant tuberculosis contacts: evidence-based recommendations. Dubai: Center for Global Health Delivery; 2015.
335. Bamrah S, Brostrom R, Dorina F, Setik L, Song R, Kawamura LM, et al. Treatment for LTBI in contacts of MDR-TB patients, Federated States of Micronesia, 2009-2012. Int J Tuberc Lung Dis 2014;18: 912-918.

336. Denholm JT, Leslie DE, Jenkin GA, Darby J, Johnson PD, Graham SM, et al. Long-term follow-up of contacts exposed to multidrug-resistant tuberculosis in Victoria, Australia, 1995-2010. Int J Tuberc Lung Dis 2012;16:1320-1325.

337. Adler-Shohet FC, Low J, Carson M, Girma H, Singh J. Management of latent tuberculosis infection in child contacts of multidrug-resistant tuberculosis. Pediatr Infect Dis J 2014;33:664-666.

338. Williams B, Ramroop S, Shah P, Anderson L, Das S, Riddell A, et al. Management of pediatric contacts of multidrug resistant tuberculosis in the United Kingdom. Pediatr Infect Dis J 2013;32:926-927.

339. Schaaf HS, Gie RP, Kennedy M, Beyers N, Hesseling PB, Donald PR. Evaluation of young children in contact with adult multidrugresistant pulmonary tuberculosis: a 30-month follow-up. Pediatrics 2002;109:765-771.

340. Horn DL, Hewlett D Jr, Alfalla C, Peterson S, Opal SM. Limited tolerance of ofloxacin and pyrazinamide prophylaxis against tuberculosis. N Engl J Med 1994;330:1241.

341. Papastavros T, Dolovich LR, Holbrook A, Whitehead L, Loeb M. Adverse events associated with pyrazinamide and levofloxacin in the treatment of latent multidrug-resistant tuberculosis. CMAJ 2002;167:131-136.

342. Younossian AB, Rochat T, Ketterer JP, Wacker J, Janssens JP. High hepatotoxicity of pyrazinamide and ethambutol for treatment of latent tuberculosis. Eur Respir J 2005;26: 462-464.

343. Alsultan A, Peloquin CA. Clinical pharmacology of the antituberculosis drugs. In: Davies PDO, Gordon SB, Davies G, editors. Clinical tuberculosis. New York, NY: CRC Press; 2014. pp. 224-243.

344. Handbook of anti-tuberculosis agents: introduction. Tuberculosis (Edinb) 2008;88:85-86. 